\title{
A catalogue of Paschen-line profiles in standard stars ${ }^{\star}$
}

\author{
W. Huang ${ }^{1,2, \star \star}$, G. Wallerstein ${ }^{1,2}$, and M. Stone ${ }^{1}$ \\ ${ }^{1}$ Department of Astronomy, University of Washington, Box 351580, Seattle, WA 98195-1580, USA \\ e-mail: wall@astro.washington.edu \\ 2 Visiting Astronomer, Dominion Astrophysical Observatory, Herzberg Institute for Astrophysics, Victoria, B.C., Canada
}

Received 12 June 2012 / Accepted 27 August 2012

\begin{abstract}
We have assembled an atlas of line profiles of the Paschen Delta $(\mathrm{P} \delta)$ line at $10049 \AA$ for the use of stellar modelling. For a few stars we have substituted the Paschen Gamma (P $\gamma$ ) line at $10938 \AA$ because the $\mathrm{P} \delta$ line blends with other features. Most of the targets are standard stars of spectral types from B to M. A few metal-poor stars have been included. For many of the stars we have also observed the Hydrogen Alpha $(\mathrm{H} \alpha)$ line so as to compare the profiles of lines originating from the meta-stable $n=2$ level with lines originating from the $n=3$ level. The greatest difference in line profile is found for high luminosity and cool stars where the departures from local thermodynamic equilibrium (LTE) in the population of the $n=2$ level is expected to be the greatest.

For a few stars, sample line profiles have been calculated in the LTE approximation to demonstrate the usefulness of the tabulated and displayed catalogue.
\end{abstract}

Key words. line: profiles - atlases - stars: early-type - stars: late-type

\section{Introduction}

The theory of model stellar atmospheres dates from the planeparallel, single-layer models of Schuster $(1902,1905)$ and Schwarzschild (1906). In 1939 two very important papers on stellar atmospheres were published by Spitzer and by Struve, Wurm, and Henyey. In Spitzer's (1939) paper on the atmospheres of $\mathrm{M}$ supergiants he noted that an excitation temperature of $17000 \mathrm{~K}$ was necessary to explain the strength of the $\mathrm{H} \alpha$ absorption line in $\alpha$ Ori even though the effective temperature of the star was about $3400 \mathrm{~K}$. In Fig. 1, we show our recent spectrum of $\alpha$ Ori with a resolving power of about 30000 . The observed $\mathrm{H} \alpha$ line (Fig. 1a) is prominent and does not appear in the spectrum as modelled with a standard atmosphere in local thermodynamic equilibrium (LTE), $\alpha$ Ori's $\mathrm{P} \delta$ line (Fig. 1b) is dominated by a blend of neutral lines, and its $\mathrm{P} \gamma$ line (Fig. 1c) is obscured by a molecular band. Struve et al. (1939) called attention to the important fact that the 2 s level from which the Balmer lines arise is meta-stable with a very small probability for the $2 \mathrm{~s} \rightarrow 1 \mathrm{~s}$ transition. This may result in an over-population of the $2 \mathrm{~s}$ level as compared with what is expected by the Boltzmann distribution in LTE. We quote them as follows, "It is tempting to explain the great strength of the Balmer absorption lines in supergiants of late type as a consequence of the metastability of the $2 \mathrm{~s}$ level. However, it is premature to discuss this matter further until accurate measurements of the Paschen lines have been made".

Most early work on stellar atmospheres was based on the assumption of LTE in which the relative populations of the atomic levels are controlled by the local temperature and the assumption that the radiation field is also dependent on the local temperature (Unsöld \& Weidemann 1955). In succeeding years the degree of

\footnotetext{
* The reduced observed spectra are only available at the CDS via anonymous ftp to cdsarc.u-strasbg. fr (130.79.128.5) or via http://cdsarc.u-strasbg.fr/viz-bin/qcat?]/A+A/547/A62 $\star \star$ Current address: Brion Technologies Inc., 4211 Burton Dr., Santa Clara, CA 95054, USA; e-mail: wenj in . huang@asml .com
}

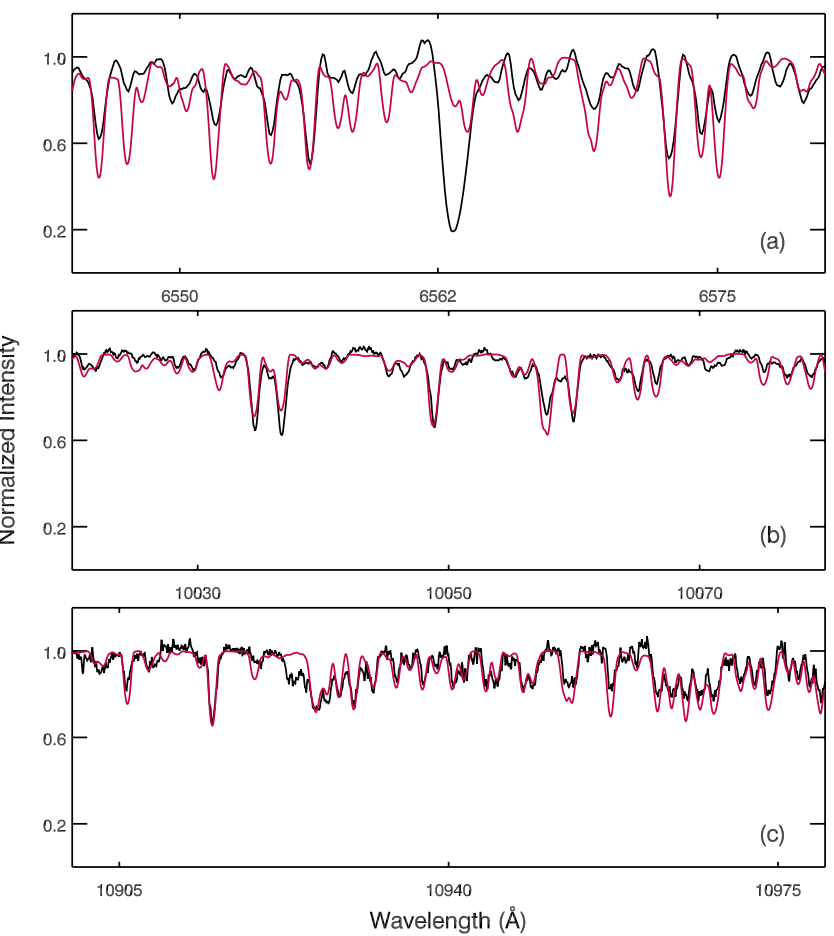

Fig. 1. Profiles for $\mathrm{H} \alpha, \mathrm{P} \delta$, and $\mathrm{P} \gamma(\mathbf{a})$, b) and c) respectively) in HD 39801 ( $\alpha$ Ori) M2 Iab. The black line is the observed spectrum and the red line is the model spectrum.

realism of model atmospheres has steadily increased, first, with sophistically calculated gradients of various physical quantities (Mihalas \& Athay 1973), through models with extended atmospheres, to the most recent models with surface fluctuations of all relevant quantities. The most recent models include surface variations associated with large scale convection and granulation 
as well as departures from LTE in their excitation and ionization equilibria (Asplund 2005).

As noted by Struve et al. (1939), in real stars there is a special problem with the assumption of LTE because the radiative transition from the $2 \mathrm{~s}$ level of hydrogen to the $1 \mathrm{~s}$ ground state is forbidden. This means that the population of the $2 \mathrm{~s}$ level must be controlled by radiative and collisional transitions with other excited levels, but transitions between the $2 \mathrm{~s}$ and $1 \mathrm{~s}$ levels are strongly inhibited. At high densities collisions are effective so that the population ratios among the $n>2$ levels approach equilibrium but the relative populations between $n=1$ and $n=2$ deviate from LTE. The populations of all levels are influenced by recombination and ionization, especially in the hotter stars. There is no question as to the presence of non-LTE (NLTE) effects; the only question is one of degree. The Paschen lines which originate from the $n=3$ level are expected to be closer to LTE than are the Balmer lines. To investigate the importance of these effects it is helpful to observe the Paschen (and higher) lines and to compare their line profiles with the predictions of atmospheric models. An additional consideration is the increased Stark effect for the Paschen lines as compared to the Balmer lines because Stark broadening increases with higher quantum numbers. Hence, the Stark broadening for the Paschen lines is expected to dominate over other broadening mechanisms.

\section{Observations}

With these factors in mind we have been observing a wide variety of stars at both $\mathrm{H} \alpha$ and $10049 \AA \mathrm{P} \delta$. The data have been obtained almost entirely with the Coudé spectrograph of the $1.2-\mathrm{m}$ telescope at the Dominion Astrophysical Observatory (DAO) of the Hertzberg Institute of Astrophysics at Victoria British Columbia. Their 1.2-m telescope with a single order Coudé spectrograph is the ideal instrument for a spectroscopic survey of bright stars in a limited wavelength region since it does not suffer from the rapidly varying continuum in each short order of an echelle system. The 96 -inch camera provides a resolving power of about 30000 over $250 \AA$ at each setting of the first order of the grating. The detector is a SITE 4 CCD with 24 micron pixels. There is a low level of fringing in the near infrared that can be removed by flat-fielding to a remaining noise level of about $1 \%$. Since the stars are bright, the remaining source of noise is proportional to the square-root of the number of electrons per pixel and to absorption by the earth's atmosphere. The latter can be largely eliminated by division of a rapidly rotating bright hot star, though caution must be used since almost all such stars have their own hydrogen lines. For broad lines whose H-line profiles are defined by many pixels the noise level is unimportant. Wavelength calibration in the near infrared can be difficult due to the paucity of emission lines in most comparison arcs. This was readily overcome by observing the ThAr spectrum in the second order and simply multipying the wavelengths so calibrated by exactly 2 .

For a few stars of types $\mathrm{K}$ and $\mathrm{M}$ there is an FeI line that blends with $\mathrm{P} \delta$ and the latter is quite weak. Since these stars are very bright in the near IR it was possible to observe the brighter ones with the SITE 4 at the P $\gamma$ line at $10938 \AA$. The $10830 \AA$ line of HeI is present in some of those spectra.

To overcome the problem of the FeI blend with the $10049 \AA$ line in the cooler stars we included some metal-poor stars. Most of those stars are too faint for the 1.2-m telescope so they were also observed with the Apache Point Observatory 3.5-m echelle. In Table 1 we list the stars for which useful spectra were obtained at the DAO. We list the metal-poor stars observed at the Apache Point Observatory in Table 2. In both tables the columns are selfexplanatory. The $M_{v}$ values are derived from the parallaxes measured by Hipparcos (van Leeuwen 2007) if the measured parallax is at least 3 times its quoted probable error. Actually, most of the parallaxes are at least 5 times their quoted error. An asterisk is shown for stars whose parallax lies between 3.0 and 5.0 times its uncertainty. No $M_{v}$ value is shown for stars with parallaxes of greater uncertainty.

\section{The line profiles}

The primary purpose of this paper is to make available accurate line profiles of selected Paschen lines so they may be used in connection with modelling stellar atmospheres. In addition, the Paschen lines may be used for calibrating the temperature scale and analysing the chemical composition of stellar atmospheres where minimizing the influence of NLTE is helpful. In a series of tables we present the line profiles as observed. Most of the stars are represented by the P $\delta$ line at $10049 \AA$. In a few stars, especially stars of type $\mathrm{K}$ and $\mathrm{M}$, the FeI line blends with $\mathrm{P} \delta$. As mentioned above, we have observed the $\mathrm{P} \gamma$ line at $10938 \AA$ whenever possible in addition to including the metal-poor stars with weakened FeI lines. For many of the stars we also obtained $\mathrm{H} \alpha$ profiles which we show for comparison with the Paschen line profiles. In Table 3 we list the wavelength, excitation of the lower level, and the transition probability of the three lines in this study. Our complete digital spectra have been deposited with the Centre de Données astronomiques de Strasbourg for access to all astronomers. To provide a complete atlas of our observations we have included all of our line profiles as figures, and we illustrate a variety of line profiles for a wide range of spectral types.

\section{LTE profile comparisons}

We also carried out calculations of $\mathrm{H} \alpha$ and $\mathrm{P} \delta$ profiles for some stars using the Kurucz models in LTE (Kurucz 1993; Sbordone et al. 2004; Sbordone 2005), the ATLAS atmospheric model production code, and the SYNTHE spectral synthesis code (Kurucz 1993; Sbordone et al. 2004). The Stark broadened profiles in the SYNTHE code are "based on the quasi-static Griem theory with parameters adjusted in such a way that profiles from the Griem theory fit the (Vidal et al. 1973) profiles of the first members of the Lyman and Balmer series" (Cowley \& Castelli 2002). Stellar parameters for profile modelling (see Table 4) were obtained from references in the online astronomical database SIMBAD with favorable weight given to the data of Fulbright (2000). Further details about the tools for atmosphere model preparation and line synthesis can be found online at the ATLAS, WIDTH and SYNTHE GNU Linux port ${ }^{1}$ Home page and under the Download page.

The figures show the synthetic LTE profiles plotted in red and the observed spectra in black.

\subsection{Comments on selected stars}

\subsubsection{Spectral sequence at high luminosity with LTE profiles}

HD 37128, B0 Iab: H $\alpha$ (Fig. 2a) shows a typical P Cygni profile while $\mathrm{P} \delta$ (Fig. $2 \mathrm{~g}$ ) is symmetric.

http://atmos.obspm.fr/ 
Table 1. Standard stars observed.

\begin{tabular}{|c|c|c|c|c|c|c|c|c|c|}
\hline \multirow[t]{2}{*}{ HD } & \multirow[t]{2}{*}{ Sp. type } & \multirow[t]{2}{*}{$M_{V}$} & \multirow[t]{2}{*}{$B-V$} & \multicolumn{3}{|c|}{ Date of observation } & \multirow[t]{2}{*}[\mathrm{Fe}/\mathrm{H}]{} & \multirow[t]{2}{*}[\mathrm{Fe}/\mathrm{H}]{ reference } & \multirow[t]{2}{*}{ Figure } \\
\hline & & & & $\mathrm{H} \alpha$ & $\mathrm{P} \delta$ & $\mathrm{P} \gamma$ & & & \\
\hline 37128 & B0Iab & $-7.21^{*}$ & -0.19 & Feb. 5, 09 & Feb. 5, 09 & $\ldots$ & $\ldots$ & $\ldots$ & 7 \\
\hline 40111 & B0.5II & -3.59 & -0.09 & Feb. 4, 09 & Feb. 5, 09 & $\ldots$ & $\ldots$ & $\ldots$ & 8 \\
\hline 91316 & B1Iab & $-7.31^{*}$ & -0.15 & Dec. 5,08 & Feb. 3, 09 & $\ldots$ & -0.89 & 1 & 9 \\
\hline 2905 & B1Iae & $-6.47^{*}$ & 0.09 & Feb. 5, 09 & Feb. 5, 09 & $\ldots$ & $\ldots$ & $\ldots$ & 10 \\
\hline 24398 & B1Ib & -3.91 & 0.08 & Feb. 5, 09 & Feb. 5, 09 & $\ldots$ & $\ldots$ & $\ldots$ & 11 \\
\hline 116658 & B1III-IV & -3.53 & -0.13 & $\ldots$ & Jul. 1, 08 & $\ldots$ & $\ldots$ & $\ldots$ & 12 \\
\hline 41117 & B2Iae & -4.02 & 0.21 & Feb. 5, 09 & Feb. 5, 09 & $\ldots$ & $\ldots$ & $\ldots$ & 13 \\
\hline 206165 & B2Ib & $\ldots$ & 0.22 & Feb. 5, 09 & & $\ldots$ & -0.33 & 1 & 14 \\
\hline 886 & B2IV & -2.64 & -0.23 & Dec. 4,08 & Feb. 3, 09 & $\ldots$ & -0.34 & 1 & 15 \\
\hline 198478 & B2.5Ia & -4.34 & 0.31 & Dec. 4, 08 & Jul. 2, 08 & $\ldots$ & -0.23 & 1 & 16 \\
\hline 35708 & B2.5IV & -1.58 & -0.14 & Dec. 5, 08 & Feb. 3, 09 & $\ldots$ & $\ldots$ & $\ldots$ & 17 \\
\hline 14143 & B3I & $\ldots$ & 0.5 & Feb. 4, 09 & $\ldots$ & $\ldots$ & $\ldots$ & $\ldots$ & 18 \\
\hline 14134 & B3Ia & $\ldots$ & 0.44 & Feb. 4, 09 & $\ldots$ & $\ldots$ & $\ldots$ & $\ldots$ & 19 \\
\hline 11415 & B3III & -2.19 & -0.12 & Dec. 4, 08 & Feb. 3, 09 & $\ldots$ & $\ldots$ & $\ldots$ & 20 \\
\hline 160762 & B3IV & -1.97 & -0.17 & Dec. 4,08 & Jul. 2, 08 & $\ldots$ & -0.4 & 2 & 21 \\
\hline 36371 & B4Ib & $\ldots$ & 0.25 & Dec. 5,08 & Feb. 3, 09 & $\ldots$ & $\ldots$ & $\ldots$ & 22 \\
\hline 15497 & B6Ia & $\ldots$ & 0.64 & Feb. 4, 09 & $\ldots$ & $\ldots$ & $\ldots$ & $\ldots$ & 23 \\
\hline 155763 & B6III & -1.88 & -0.1 & Jul. 4, 08 & Jul. 2, 08 & $\ldots$ & -0.95 & 3 & 24 \\
\hline 3240 & B7III & -1.03 & -0.1 & Dec. 4, 08 & $\ldots$ & $\ldots$ & $\ldots$ & $\ldots$ & 25 \\
\hline 69686 & B8 & $-1.10^{*}$ & -0.52 & Feb. 4, 09 & $\ldots$ & $\ldots$ & $\ldots$ & $\ldots$ & 26 \\
\hline 34085 & B8Iab & -6.92 & -0.03 & Dec. 5, 08 & Feb. 3, 09 & $\ldots$ & $\ldots$ & $\ldots$ & 27 \\
\hline 14322 & B8Ib & $\ldots$ & 0.26 & Feb. 4, 09 & $\ldots$ & $\ldots$ & $\ldots$ & $\ldots$ & 28 \\
\hline 63975 & B8II & -1.32 & -0.11 & Feb. 4, 09 & Feb. 5, 09 & $\ldots$ & $\ldots$ & $\ldots$ & 29 \\
\hline 21291 & B9Ia & $-4.56^{*}$ & 0.34 & Feb. 5, 09 & Feb. 5, 09 & $\ldots$ & $\ldots$ & $\ldots$ & 30 \\
\hline 212593 & B9Iab & $-4.58^{*}$ & 0.07 & Feb. 5, 09 & & $\ldots$ & $\ldots$ & $\ldots$ & 31 \\
\hline 46300 & A0Ib & $\ldots$ & 0 & Feb. 5, 09 & Feb. 5, 09 & $\ldots$ & -0.3 & 4 & 32 \\
\hline 87737 & $\mathrm{~A} 0 \mathrm{Ib}$ & -4.45 & -0.03 & Dec. 5, 08 & Feb. 3, 09 & $\ldots$ & -0.05 & 4 & 33 \\
\hline 47105 & A0IV & -0.70 & 0.03 & Dec. 5, 08 & Feb. 3, 09 & $\ldots$ & 0.08 & 5 & 34 \\
\hline 25642 & A0IVn & -1.28 & 0.02 & Dec. 5, 08 & $\ldots$ & $\ldots$ & $\ldots$ & $\ldots$ & 35 \\
\hline 172167 & A0V & 0.66 & 0 & Jul. 4, 08 & Jul. 1, 08 & Sep. 30, 09 & -0.43 & 6 & 36 \\
\hline 34787 & A0Vne & 0.18 & -0.02 & Dec. 5, 08 & $\ldots$ & $\ldots$ & $\ldots$ & $\ldots$ & 37 \\
\hline 14433 & A1Ia & $\ldots$ & 0.58 & Feb. 4, 09 & $\ldots$ & $\ldots$ & $\ldots$ & $\ldots$ & 38 \\
\hline 214994 & A1IV & 0 & -0.01 & Dec. 4,08 & $\ldots$ & $\ldots$ & 0.42 & 7 & 39 \\
\hline 14489 & A2Ia & $-4.73^{*}$ & 0.29 & Feb. 4, 09 & $\ldots$ & $\ldots$ & -0.26 & 4 & 40 \\
\hline 197345 & A2Iae & -6.89 & 0.09 & Jul. 4, 08 & Jul. 1, 08 & $\ldots$ & 0.15 & 8 & 41 \\
\hline 50019 & A3III & -0.18 & 0.1 & Dec. 5, 08 & Feb. 3, 09 & $\ldots$ & $\ldots$ & $\ldots$ & 42 \\
\hline 17378 & A5Ia & $\ldots$ & 0.75 & Dec. $26,10^{\dagger}$ & Dec. $26,10^{\dagger}$ & $\ldots$ & -0.07 & 9 & 43 \\
\hline 2628 & A7III & 1.29 & 0.24 & Dec. 4, 08 & $\ldots$ & $\ldots$ & -0.24 & 6 & 44 \\
\hline 31964 & A8Iab & $\ldots$ & 0.49 & Feb. 4, 09 & Feb. 3, 09 & Sep. 30, 09 & $\ldots$ & $\ldots$ & 45 \\
\hline 182835 & F2Iab & $-4.92^{*}$ & 0.52 & Dec. 4,08 & Jul. 1, 08 & $\ldots$ & -0.03 & 9 & 46 \\
\hline 20902 & F5Iab & -4.06 & 0.47 & Dec. 5, 08 & Feb. 3, 09 & Aug. 25,10 & -0.07 & 9 & 47 \\
\hline 213306 & F5Iab & -2.84 & 0.74 & $\ldots$ & Jul. 2, 08 & $\ldots$ & $\ldots$ & $\ldots$ & 48 \\
\hline 187929 & F6Iab & $\ldots$ & 0.74 & $\ldots$ & Jul. 2, 08 & $\ldots$ & 0.01 & 10 & 49 \\
\hline 33564 & F6V & 3.59 & 0.45 & Dec. 5, 08 & $\ldots$ & $\ldots$ & 0.21 & 11 & 50 \\
\hline 215648 & F7V & 3.25 & 0.49 & Dec. 4, 08 & $\ldots$ & $\ldots$ & -0.35 & 12 & 51 \\
\hline 18391 & G0Ia & $\ldots$ & 1.87 & Dec. $26,10^{\dagger}$ & Dec. $26,10^{\dagger}$ & $\ldots$ & $\ldots$ & $\ldots$ & 52 \\
\hline 204867 & G0Ib & -3.04 & 0.83 & Dec. 4,08 & Jul. 1, 08 & $\ldots$ & 0.1 & 9 & 53 \\
\hline 209750 & $\mathrm{G} 2 \mathrm{Ib}$ & -2.92 & 0.96 & Dec. 4,08 & Jul. 2, 08 & $\ldots$ & 0.03 & 9 & 54 \\
\hline 188727 & G5Ibv & & 0.88 & Dec. 4,08 & Jul. 2, 08 & $\ldots$ & 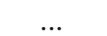 & $\ldots$ & 55 \\
\hline 71369 & G5III & -0.19 & 0.83 & Dec. 5, 08 & Feb. 3, 09 & $\ldots$ & -0.16 & 13 & 56 \\
\hline 77912 & G8Ib-II & -2.27 & 1.02 & Dec. 5, 08 & Feb. 3, 09 & $\ldots$ & 0.38 & 14 & 57 \\
\hline 62345 & G8IIIa & 0.54 & 0.93 & Dec. 5, 08 & Feb. 3, 09 & $\ldots$ & -0.02 & 13 & 58 \\
\hline 6833 & G9III & 0.31 & 1.14 & Dec. $26,10^{\dagger}$ & Dec. $26,10^{\dagger}$ & $\ldots$ & -0.89 & 15 & 59 \\
\hline 124897 & K1.5III & -0.15 & 1.23 & Jul. 4, 08 & Jul. 2, 08 & $\ldots$ & -0.52 & 16 & 60 \\
\hline 206778 & $\mathrm{~K} 2 \mathrm{Ib}$ & -4.08 & 1.56 & Dec. 4,08 & Jul. 1, 08 & Sep. 30, 09 & -0.18 & 13 & 61 \\
\hline 85503 & K2III & 1.15 & 1.22 & $\ldots$ & Jul. 2, 08 & $\ldots$ & 0.29 & 13 & 62 \\
\hline
\end{tabular}

Notes. ${ }^{(*)}$ The parallax for this star lies between 3 and 5 times its probable error. ${ }^{(\dagger)}$ Data obtained at the Apache Point Observatory.

References. (1) Gies \& Lambert (1992); (2) Peters \& Aller (1970); (3) Smith \& Dworetsky (1993); (4) Venn (1995); (5) Hill (1995); (6) Erspamer \& North (2003); (7) Hui-Bon-Hoa (2000); (8) Albayrak (2000); (9) Lyubimkov et al. (2010); (10) Luck \& Lambert (1981); (11) Gonzalez et al. (2010); (12) Fuhrmann (2008); (13) Hekker \& Meléndez (2007); (14) Fernandez-Villacanas et al. (1990); (15) Mishenina \& Kovtyukh (2001); (16) Meléndez et al. (2008); (17) Mallik (1998); (18) Bakos (1971); (19) McWilliam (1990); (20) Luck \& Bond (1980); (21) Carr et al. (2000); (22) Smith \& Lambert (1985). 
Table 1. continued.

\begin{tabular}{|c|c|c|c|c|c|c|c|c|c|}
\hline \multirow[t]{2}{*}{ HD } & \multirow[t]{2}{*}{ Sp. type } & \multirow[t]{2}{*}{$M_{V}$} & \multirow[t]{2}{*}{$B-V$} & \multicolumn{3}{|c|}{ Date of observation } & \multirow[t]{2}{*}[\mathrm{Fe}/\mathrm{H}]{} & \multirow[t]{2}{*}[\mathrm{Fe}/\mathrm{H}]{ reference } & \multirow[t]{2}{*}{ Figure } \\
\hline & & & & $\mathrm{H} \alpha$ & $\mathrm{P} \delta$ & $\mathrm{P} \gamma$ & & & \\
\hline 156283 & K3Iab & -2 & 1.46 & Jul. 4, 08 & Jul. 2, 08 & $\ldots$ & 0.01 & 13 & 63 \\
\hline 17506 & K3Ib & -3.26 & 1.68 & Feb. 4, 09 & $\ldots$ & $\ldots$ & 0.09 & 17 & 64 \\
\hline 44537 & K5Iab & $\ldots$ & 1.96 & Feb. 5, 09 & Feb. 5, 09 & $\ldots$ & 0.08 & 18 & 65 \\
\hline 29139 & K5III & -0.55 & 1.48 & $\ldots$ & $\ldots$ & Sep. 30, 09 & -0.15 & 16 & 66 \\
\hline 147767 & K5III & -0.78 & 1.57 & $\ldots$ & Jul. 1, 08 & $\ldots$ & -0.16 & 19 & 67 \\
\hline 42543 & M0Iab & $\ldots$ & 2.22 & Feb. 5, 09 & Feb. 5, 09 & $\ldots$ & -0.42 & 20 & 68 \\
\hline 6860 & MOIII & -1.74 & 1.58 & Dec. 4,08 & Jul. 2, 08 & Sep. 30,09 & -0.04 & 21 & 69 \\
\hline 14330 & M1sd & $\ldots$ & 2.07 & Dec. $26,10^{\dagger}$ & Dec. $26,10^{\dagger}$ & 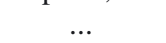 & $\ldots$ & $\ldots$ & 70 \\
\hline 206936 & M2Ia & $\ldots$ & 2.26 & Aug. 25,10 & $\ldots$ & Aug. 25,10 & $\ldots$ & $\ldots$ & 71 \\
\hline 36389 & M2Iab & -4.36 & 2.07 & Feb. 5, 09 & Feb. 5, 09 & Sep. 30,09 & 0.11 & 20 & 72 \\
\hline 39801 & M2Iab & -5.42 & 1.85 & Dec. 5, 08 & Feb. 3, 09 & Sep. 30,09 & 0 & 21 & 1 \\
\hline 13136 & M2Iab-b & $\ldots$ & 2.29 & Dec. $26,10^{\dagger}$ & Dec. $26,10^{\dagger}$ & $\ldots$ & $\ldots$ & $\ldots$ & 73 \\
\hline 147749 & M2III & -1.19 & 1.64 & Jul. 4, 08 & $\ldots$ & $\ldots$ & $\ldots$ & $\ldots$ & 74 \\
\hline 217906 & M2.5II-III & -1.41 & 1.67 & Aug. 25,10 & Jul. 2, 08 & Sep. 30, 09 & -0.11 & 22 & 75 \\
\hline 14469 & M3Iab & $\ldots$ & 2.12 & $\ldots$ & Jul. 2, 08 & & & & 76 \\
\hline 42995 & M3III & -2.06 & 1.59 & $\ldots$ & $\ldots$ & Sep. 30, 09 & 0.04 & 16 & 77 \\
\hline 44478 & M3III & -1.34 & 1.62 & $\ldots$ & $\ldots$ & Sep. 30, 09 & 0 & 16 & 78 \\
\hline 172380 & M4Iab & $-2.74^{*}$ & 1.44 & Aug. 25,10 & $\ldots$ & Aug. 25,10 & $\ldots$ & $\ldots$ & 79 \\
\hline 197812 & M5Iab & $\ldots$ & 1.42 & Aug. 25,10 & $\ldots$ & Aug. 25,10 & $\ldots$ & $\ldots$ & 80 \\
\hline 156014 & M5Ib-II & -2.3 & 1.45 & Jul. 4, 08 & Jul. 1, 08 & $\ldots$ & $\ldots$ & $\ldots$ & 81 \\
\hline
\end{tabular}

Table 2. Metal-poor stars observed ${ }^{\dagger}$.

\begin{tabular}{|c|c|c|c|c|c|c|c|c|c|}
\hline \multirow[t]{2}{*}{ HD } & \multirow[t]{2}{*}{ Name } & \multirow[t]{2}{*}{ Sp. type } & \multirow[t]{2}{*}{$M_{V}$} & \multirow[t]{2}{*}{$B-V$} & \multicolumn{2}{|c|}{ Date of observation } & \multirow[t]{2}{*}[\mathrm{Fe}/\mathrm{H}]{} & \multirow[t]{2}{*}[\mathrm{Fe}/\mathrm{H}]{ reference } & \multirow[t]{2}{*}{ Figure } \\
\hline & & & & & $\mathrm{H} \alpha$ & $\mathrm{P} \delta$ & & & \\
\hline 2796 & BD-17 70 & $\mathrm{Fp}$ & & 0.67 & Nov. 21,10 & Nov. 21,10 & -2.21 & 1 & 82 \\
\hline 140283 & BD-10 4149 & F3sd & 3.47 & 0.5 & May 9, 09 & May 9, 09 & -2.38 & 2 & 83 \\
\hline 6755 & $\mathrm{BD}+60170$ & F8V & 2.14 & 0.67 & Aug. 21,10 & Aug. 21,10 & -1.47 & 1 & 84 \\
\hline 221170 & $\mathrm{BD}+294940$ & G2IV & $0.18^{*}$ & 1.02 & Aug. 21,10 & Aug. 21,10 & -2.05 & 1 & 85 \\
\hline 216143 & BD-07 5873 & G5 & $\ldots$ & 0.94 & Aug. 21,10 & Aug. 21,10 & -2.11 & 1 & 86 \\
\hline 2665 & $\mathrm{BD}+5670$ & G5IIIp & $0.36^{*}$ & 0.71 & Dec. 26,10 & Dec. 26,10 & -1.97 & 2 & 87 \\
\hline$\ldots$ & BD+44 493 & G5IV & $2.62^{*}$ & 0.52 & Dec. 26,10 & Dec. 26,10 & -3.7 & 3 & 88 \\
\hline 218732 & BD-14 6415 & $\mathrm{G} 7 \mathrm{Ib}$ & $\ldots$ & 1.66 & Nov. 21,10 & Nov. 21,10 & -1.64 & 4 & 89 \\
\hline 187111 & BD-12 5540 & G8p & $\ldots$ & 1.17 & Jul. 23, 10 & Jul. 23, 10 & -1.74 & 1 & 90 \\
\hline 122563 & BD+10 2617 & K0IIp & -0.55 & 0.9 & May 5, 09 & May 5, 09 & -2.71 & 5 & 91 \\
\hline 103095 & $\mathrm{BD}+382285$ & K0Vp & 6.77 & 0.75 & Jun. 8, 09 & Jun. 8, 09 & -1.35 & 2 & 92 \\
\hline 4306 & BD-10 155 & KIIp & $2.65^{*}$ & 0.63 & Nov. 21,10 & Nov. 21,10 & -2.52 & 1 & 93 \\
\hline 25329 & BD+34 796 & K1V & 7.33 & 0.87 & Nov. 21,10 & Nov. 21,10 & -1.84 & 6 & 94 \\
\hline 165195 & BD+03 3579 & K3p & $\ldots$ & 1.24 & Jul. 23, 10 & Jul. 23, 10 & -2.03 & 1 & 95 \\
\hline 232078 & $\mathrm{BD}+163924$ & K3IIp & $\ldots$ & 2.03 & Jul. 23, 10 & Jul. 23, 10 & -1.6 & $3 \& 4$ & 96 \\
\hline
\end{tabular}

Notes. ${ }^{(*)}$ The parallax for this star lies between 3 and 5 times its probable error. ${ }^{(\dagger)}$ Data obtained at the Apache Point Observatory.

References. (1) Mishenina \& Kovtyukh (2001); (2) Bergemann \& Gehren (2008); (3) Barzdis (2010); (4) Ito (2009); (5) Burris et al. (2000); (6) Bergemann \& Gehren (2008).

Table 3. Atomic data for hydrogen lines.

\begin{tabular}{lcccc}
\hline \hline Line & Wavelength $(\AA)$ & $\begin{array}{c}\text { Lower } \\
\text { energy } \\
\text { level }\end{array}$ & $\begin{array}{c}\text { Upper } \\
\text { energy } \\
\text { level }\end{array}$ & $\log g f$ \\
\hline $\mathrm{H} \alpha$ & 6562.80 & $2^{2} \mathrm{p}^{0}$ & $3^{2} \mathrm{~d}$ & +0.71 \\
$\mathrm{P} \delta$ & 10049.4 & $3^{2} \mathrm{~d}$ & $7^{2} \mathrm{p}^{0}$ & -0.30 \\
$\mathrm{P} \gamma$ & 10938.1 & $3^{2} \mathrm{~d}$ & $6^{2} \mathrm{p}^{0}$ & +0.00 \\
\hline
\end{tabular}

HD 46300 (13 Mon) A0 Ib: For 13 Mon there is a small difference in the profile of $\mathrm{H} \alpha$ (Fig. 2b) while the Paschen line (Fig. 2h) fits the model almost exactly.

HD 182835, F2 Iab: As for HD 46300, there is a small difference between the model and the observations in the line wings (Fig. 2c). The Paschen line fits almost exactly (Fig. 2i).
HD 209750, G2 Ib: The observed H $\alpha$ line (Fig. 2d) appears to be wider than the model, while the model and observation of the $\mathrm{P} \delta$ line (Fig. 2j) fit very well.

HD 206778, K2 Ib \& HD 42543, M0 Iab: The trend as the spectral type becomes later shows the observed $\mathrm{H} \alpha$ line (Figs. 2e, f) becoming steadily stronger as compared to the model.

\subsubsection{Sequence at high luminosity at mid-spectral types}

HD 34085 (Rigel) B8 Iab: This standard B supergiant shows a self-absorbed $\mathrm{H} \alpha$ emission line (Fig. 3a) and a symmetric $\mathrm{P} \delta$ line (Fig. 3g).

HD 17378, A5 Ia: This supergiant member of the $\mathrm{h}$ and $\chi$ Per association is a little cooler than $\alpha$ Cyg and shows an almost 
Table 4. Model hydrogen line profile parameters.

\begin{tabular}{lcccccc}
\hline \hline Figure & Star name & HD & $T_{\text {eff }}$ & $\log g$ & $V_{\mathrm{t}}\left(\mathrm{km} \mathrm{s}^{-1}\right)$ & {$[\mathrm{Fe} / \mathrm{H}]$} \\
\hline 2b \& 2h & $13 \mathrm{Mon}$ & 46300 & 9700 & 2.0 & 4.0 & -0.1 \\
6 \& 5a & Vega & 172167 & 9540 & 4.0 & 1.2 & -0.5 \\
41 & Deneb & 197345 & 9100 & 1.5 & 6.0 & 0.0 \\
2c \& 2i & $\ldots$ & 182835 & 6750 & 1.0 & 5.0 & 0.0 \\
3c \& 3i & $\alpha$ Per & 20902 & 6350 & 1.9 & 5.3 & 0.0 \\
2d \& 2j & $\ldots$ & 209750 & 5250 & 1.5 & 3.8 & 0.0 \\
$4 \mathrm{e} \& 4 \mathrm{k}$ & Groombridge 1830 & 103095 & 4950 & 4.5 & 0.7 & -1.3 \\
58 & $\ldots$ & 62345 & 4800 & 2.9 & 3.8 & -0.3 \\
95 & $\ldots$ & 165195 & 4430 & 1.2 & 1.9 & -2.1 \\
$4 \mathrm{~b} \& 4 \mathrm{~h}$ & $\ldots$ & 122563 & 4425 & 0.6 & 2.1 & -2.6 \\
$4 \mathrm{~d} \& 4 \mathrm{j}$ & Arcturus & 124897 & 4250 & 1.9 & 1.4 & -0.5 \\
$4 \mathrm{c} \& 4 \mathrm{i}$ & $\ldots$ & 232078 & 4000 & 0.3 & 2.6 & -1.6 \\
1 & $\alpha$ Ori & 39801 & 3540 & 0.0 & 2.3 & 0.0 \\
\hline
\end{tabular}

symmetric $\mathrm{H} \alpha$ line (Fig. 3b) at the time of observation with little evidence for either a wind or active chromosphere.

HD 20902 ( $\alpha$ Per) F5 Iab: The fit for $\mathrm{H} \alpha$ (Fig. 3c) is very good and for $\mathrm{P} \delta$ (Fig. 3i) is almost perfect.

HD 77912, G8 Ib-II: The strong absorption at $\mathrm{H} \alpha$ (Fig. 3d) contrasts with the weaker line at $\mathrm{P} \delta$ (Fig. 3j).

HD 44537, K5 Iab: The H $\alpha$ line (Fig. 3e) is strongly present and asymmetric. The $\mathrm{P} \delta$ line (Fig. $3 \mathrm{k}$ ) is dominated by a blend of neutral lines.

HD 156014, M5 Ib-II: Despite the late spectral type, the observed $\mathrm{H} \alpha$ profile (Fig. 3f) remains strong.

\subsubsection{Spectral type K stars}

HD 122563, K0 IIp: This very metal-poor giant, whose $[\mathrm{Fe} / \mathrm{H}]=-2.7$, shows only small deviations from the model for its $\mathrm{H} \alpha$ (Fig. 4b) and $\mathrm{P} \delta$ (Fig. 4h) lines.

HD 232078, K3 IIp: (Figs. 4c and 4i) This red giant is in a markedly retrograde orbit and is similar to globular cluster red giants. Its $T_{\text {eff }}$ of $4000 \mathrm{~K}$ is one of the lowest values for a metalpoor field star. With $[\mathrm{Fe} / \mathrm{H}]=-1.6$, the metallic lines still obscure the $\mathrm{P} \delta$ line.

HD 124897 (Arcturus) K1.5 III: For this moderately metalpoor star, the observed $\mathrm{H} \alpha$ line (Fig. 4d) is much stronger than the model profile but the $\mathrm{P} \delta$ line (Fig. 4j) fits the model very well.

HD 103095 (Groombridge 1830) K0 Vp: This well-known, metal-poor main sequence star, with a $T_{\text {eff }}$ of $5000 \mathrm{~K}$, appears to have a slightly deeper core at $\mathrm{H} \alpha$ (Fig. 4e) and shallower core at $\mathrm{P} \delta$ (Fig. 4k) than predicted.

\subsubsection{P $\gamma$ profiles}

HD 172167 (Vega) A0 V: This fundamental standard for modelling stellar atmospheres shows a very good fit between the modelled and the observed profile for $\mathrm{P} \gamma$ (Fig. 5a).

HD 31964, A8 Iab: The P $\gamma$ line is clearly shown (Fig. 5b).

HD 20902 ( $\alpha$ Per) F5 Iab: There is a great deal of blending at $10938 \AA$, but the $\mathrm{P} \gamma$ line is visible (Fig. 5c).

HD 206778, K1.5 III: The P $\gamma$ line is obscured by a molecular band (Fig. 5d).

\subsection{Additional comments on selected stars}

Figure 15, HD 886, B2 IV: This standard early B star shows symmetrical $\mathrm{H} \alpha$ and $\mathrm{P} \delta$ profiles.

Figure 41, HD 197345 (Deneb) A2 Iae: This standard supergiant shows its well-known $\mathrm{P}$ Cygni structure at $\mathrm{H} \alpha$ while its $\mathrm{P} \delta$ profile fits the model exactly.

Figure 52, HD 18391, G0 Ia: This G supergiant may be an outer member of the $\mathrm{h}$ and $\chi$ Per cluster. Its $\mathrm{H}$ lines appear to be symmetric.

Figure 69, HD 6860, M0 III: The H $\alpha$ line is clearly present while the $\mathrm{P} \delta$ line is dominated by the blend of neutral metals. There appears to be almost no feature at $\mathrm{P} \gamma$. A weak molecular band may be interfering.

Figure 71, HD 206936 ( $\mu$ Cep) M2 Ia: This supergiant may be even more luminous than $\alpha$ Ori. Its $\mathrm{H} \alpha$ line is similar to that of $\alpha$ Ori. There is no evidence for the $\mathrm{P} \gamma$ line though the molecular band seen in $\alpha$ Ori is weaker in $\mu$ Cep.

Figure 79, HD 172380 (XY Lyr) M4 Iab: H $\alpha$ is clearly present while $\mathrm{P} \gamma$ is not evident. The molecular band seen in $\alpha$ Ori is not present.

Figure 80, HD 197812 (U Del) M5 Ia: A weak molecular band appears in the star and in most stars of type M5 or later very close to the position of $\mathrm{H} \alpha$. No line is visible at $\mathrm{P} \gamma$.

Figure 85, HD 221170, G2 IV: $\mathrm{H} \alpha$ is symmetrical and much stronger than $\mathrm{P} \delta$.

Figure 94, HD 25329, K1 V: This subdwarf is significantly metal-poor and is similar to HD 103095. For both stars $\mathrm{H} \alpha$ is much stronger than $\mathrm{P} \delta$.

Figure 95, HD 165195, K3 p: This star's metallicity is similar to giant branch stars in globular clusters and shows weak $\mathrm{H} \alpha$ emission that is usually associated with mass loss.

\section{Discussion}

Because the NLTE effects are much reduced in main-sequence stars, we expect that the synthesized LTE profiles should fit well with both $\mathrm{H} \alpha$ and $\mathrm{P} \delta$ observation. The result in Fig. 6 (for Vega, A0 V) is a very good example of this case, the LTE model $\left(T_{\text {eff }}=9540 \mathrm{~K}, \log _{\mathrm{g}}=4.0\right)$ fit both $\mathrm{H} \alpha$ and $\mathrm{P} \delta$ well. But for the A0 supergiant, 13 Mon (HD 46300, A0 Ib), an LTE model can not match them simultaneously. When the LTE model matches the observed $\mathrm{P} \delta$ well (see Fig. 2h), it overestimates the strength of $\mathrm{H} \alpha$ (see Fig. 2b). 


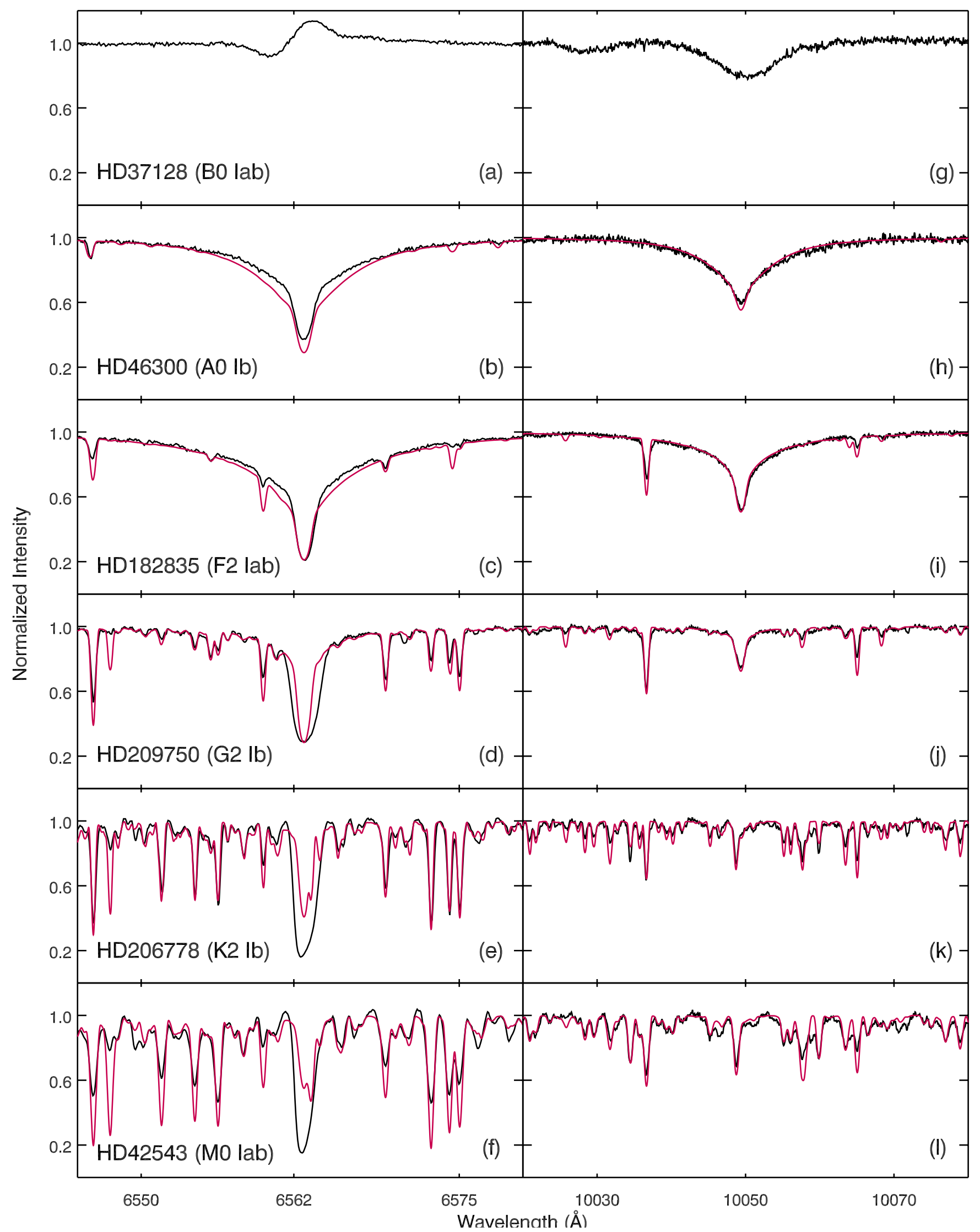

Fig. 2. Profiles of $\mathrm{H} \alpha \mathbf{a})-\mathbf{f}$ ) and $\mathrm{P} \delta \mathbf{g}$ )-l) for selected stars of high luminosity. 
W. Huang et al.: A catalogue of Paschen-line profiles

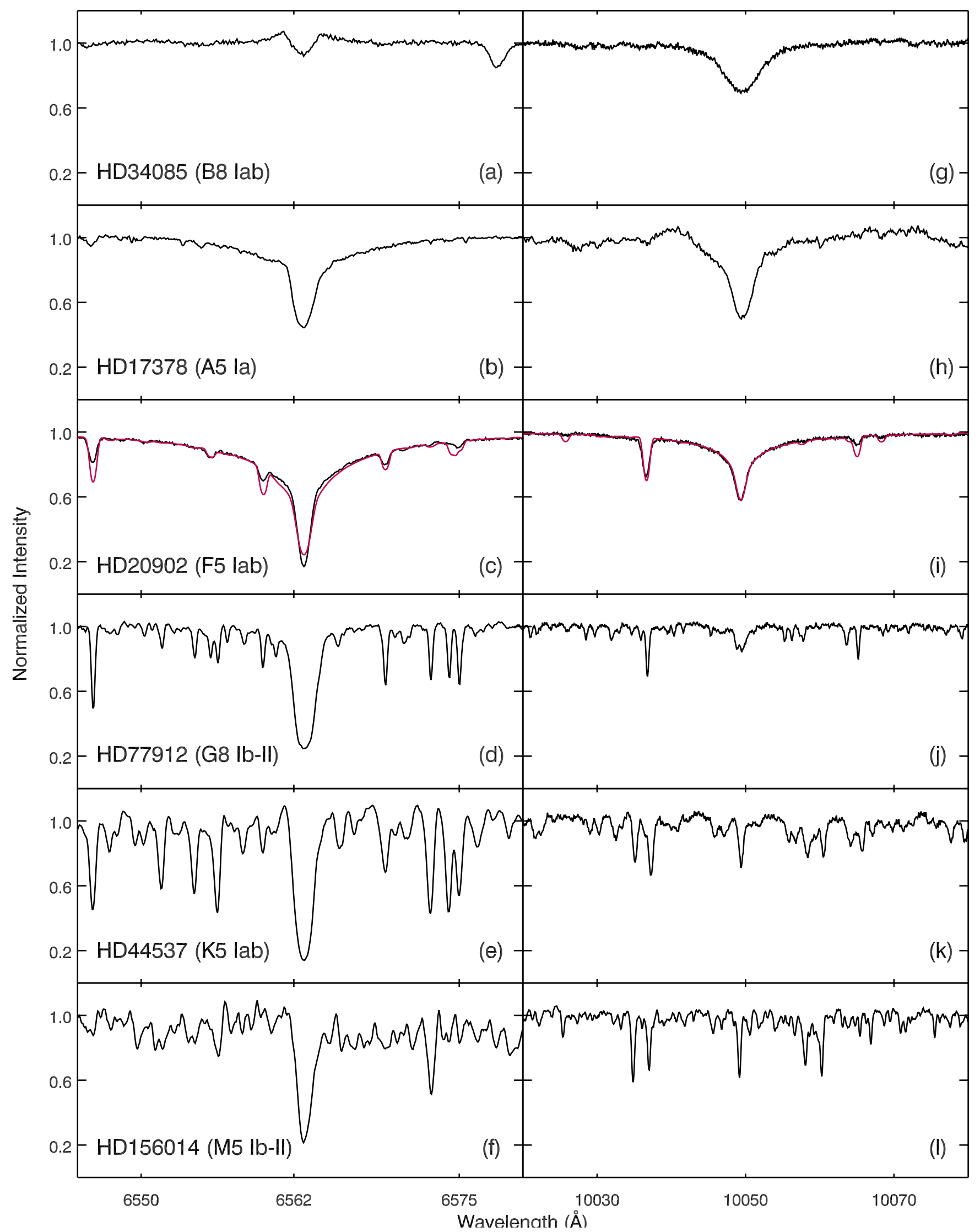

Fig. 3. Profiles of $\mathrm{H} \alpha \mathbf{a})-\mathbf{f}$ ) and $\mathrm{P} \delta \mathbf{g}$ )-l) for selected stars of high luminosity. 


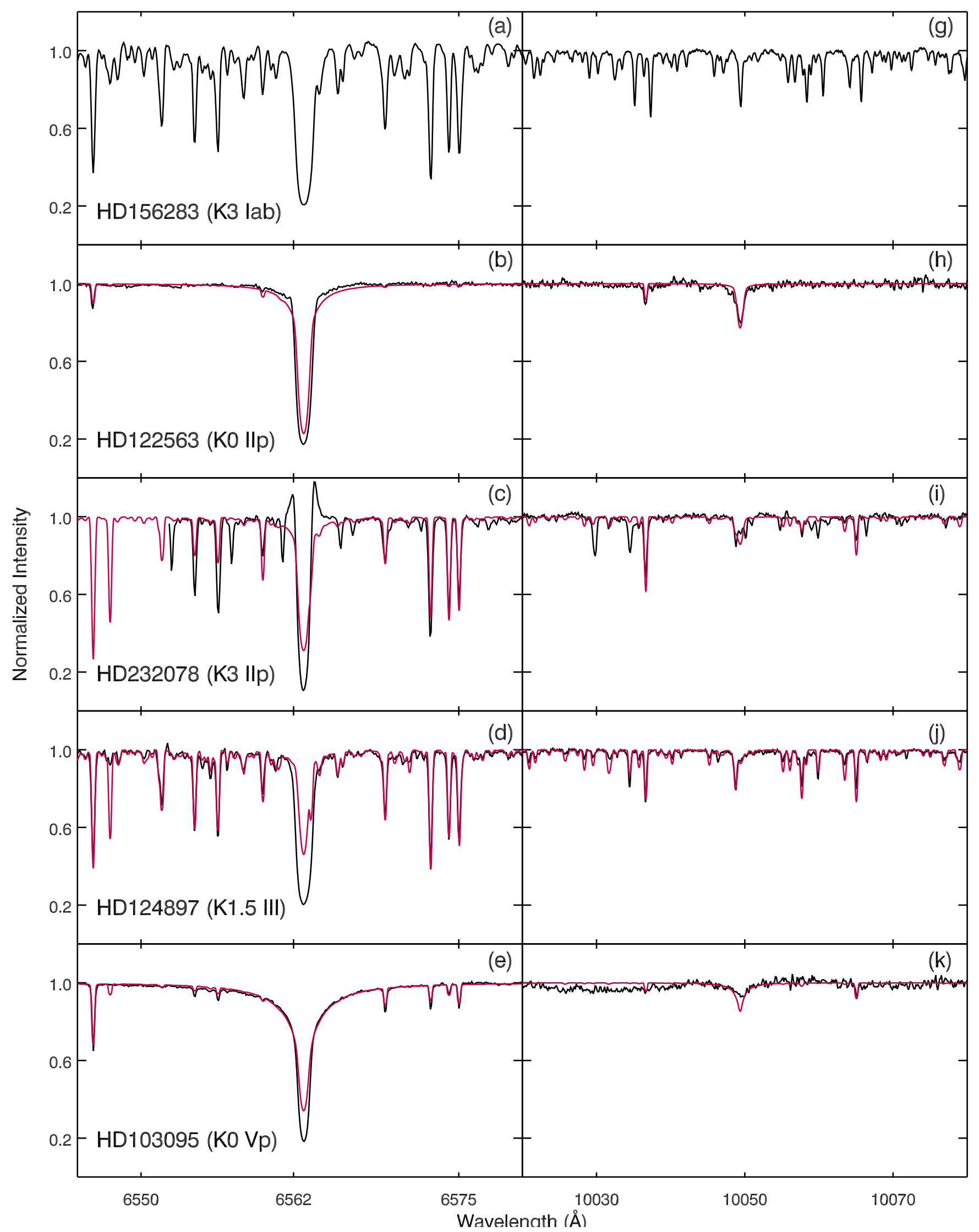

Fig. 4. Profiles of $\mathrm{H} \alpha \mathbf{a}$ )-e) and $\mathrm{P} \delta \mathbf{g}$ )-k) for selected spectral type K stars. 


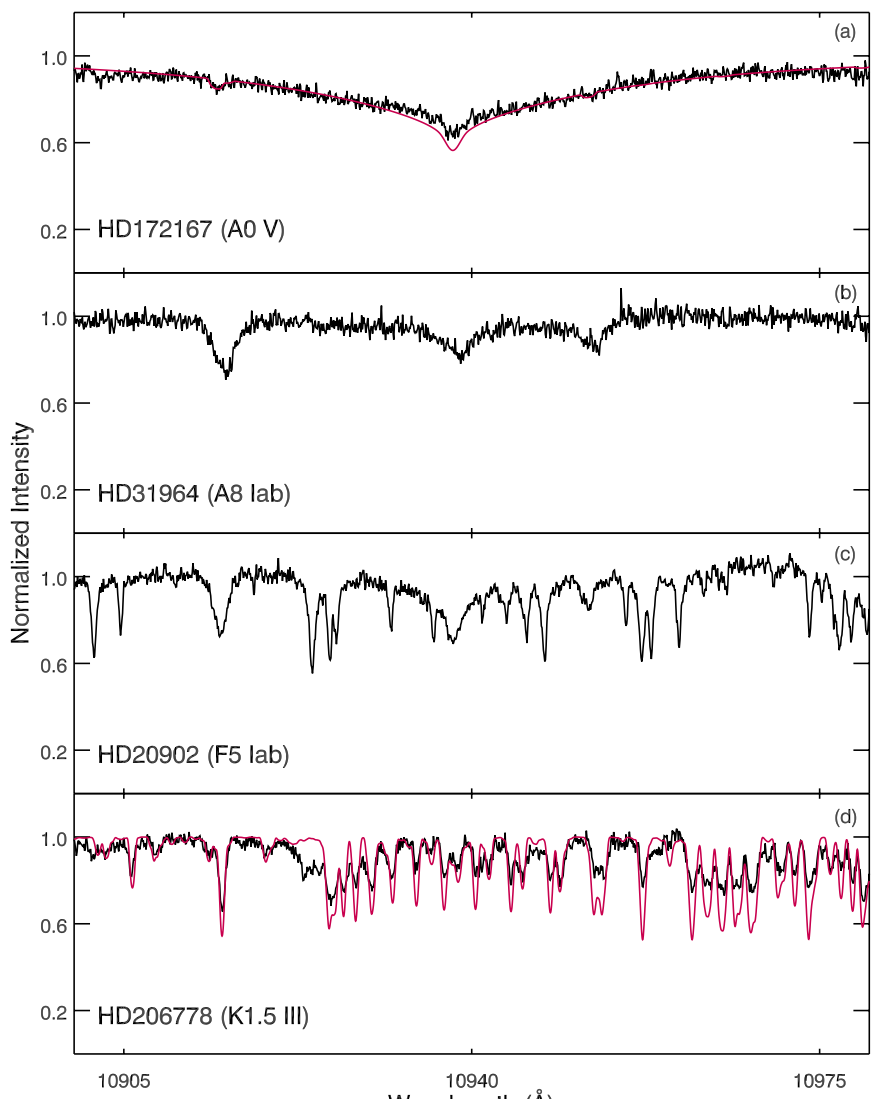

Fig. 5. Profiles of $\mathrm{P} \gamma$ for select stars.

For super giants with lower temperature (but hotter than F5), we continue to see a similar difference between $\mathrm{H} \alpha$ and $\mathrm{P} \delta$ fittings which, however, become smaller towards lower temperatures (see Figs. 2c and 2i). The difference disappears around the spectral type of F5 ( $\alpha$ Per, F5 Iab, in Figs. 3c and 3i, for example).

When the effective temperature drops to that of $\mathrm{G}$ type, the LTE models once again show their failure to reproduce the observed $\mathrm{H} \alpha$ profiles. In contrast to the case of hot luminous stars (Figs. 6, 2b, 2h), this time the LTE model profiles of $\mathrm{H} \alpha$ are weaker than the observed line profiles (Fig. 2d).

In the low temperature regime of $\mathrm{K}$ and $\mathrm{M}$ type giants/supergiants, the $\mathrm{H} \alpha$ profiles predicted by the LTE models become very weak while the observed profiles maintain their strength to appear almost the same as this of G stars. Arcturus, a moderately metal-poor star, has a much stronger $\mathrm{H} \alpha$ line than modelled (Fig. 4d). The very metal-poor giant HD 122563 also shows a weaker LTE-modelled $\mathrm{H} \alpha$ profile (Fig. 4b), as does the metal-poor K0 dwarf Groombridge 1830 (Fig. 4e). In later type $\mathrm{M}$ giants, the spectrum is dominated by $\mathrm{TiO}$ bands and $\mathrm{H} \alpha$ disappears. We should note that, for those cool luminous stars with detectable $\mathrm{P} \delta$, the synthetic $\mathrm{P} \delta$ LTE profiles still provide a good fit to the observed spectra (Figs. 4h and $4 \mathrm{j}$ ).

When we compare the LTE model profiles with observations, the differences between $\mathrm{H} \alpha$ and $\mathrm{P} \delta$ are noticeable in both hot stars and, more significantly, in cool luminous stars. It is clear that the forbidden transition between the $1 \mathrm{~s}$ and $2 \mathrm{~s}$ levels plays a key role here. The atomic hydrogen population in the $2 \mathrm{~s}$ level is dynamically balanced through two channels. The first channel is the collisional, non-radiative transition. The second is the two-step radiative transit through the np levels ( $1 \mathrm{~s} \leftrightarrow n \mathrm{p} \leftrightarrow 2 \mathrm{~s}$, $n>2$ ). In a low density environment such as the upper atmo-

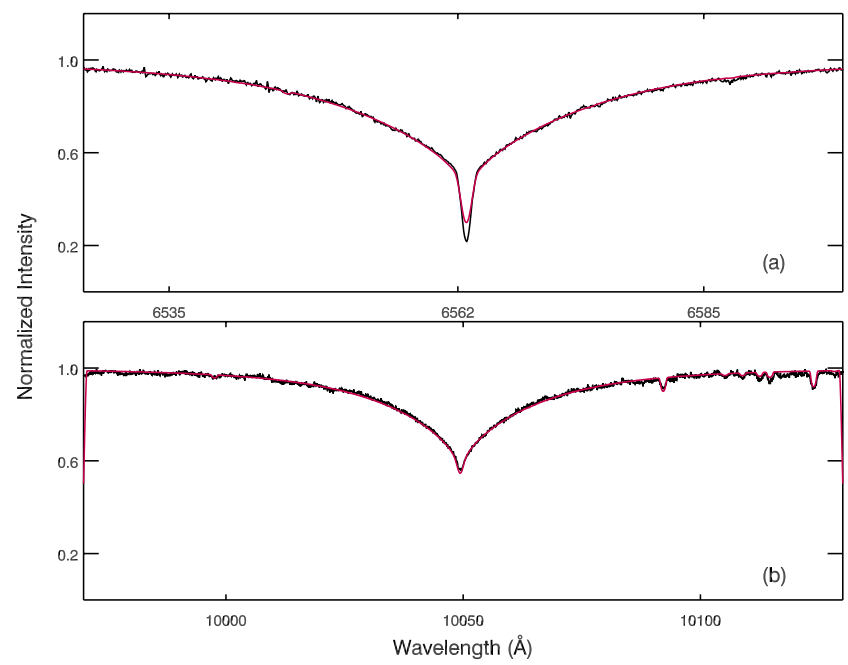

Fig. 6. HD 172167 (Vega) A0 V: except for a very small deviation at the center of the $\mathrm{H} \alpha$ profile, this fundamental standard for modelling stellar atmospheres shows a very good fit between the modelled and the observed profiles for $\mathrm{H} \alpha$ and $\mathrm{P} \delta$.

sphere of a very luminous giant star, collisional transition contributions to the 2 s level decrease due to the low density. Our results seem to suggest that in the high temperature regime (A and early $\mathrm{F}$ type stars), this decrease in collisional transitions causes the $2 \mathrm{~s}$ population to fall short of the LTE-predicted population. In the low temperature regime ( $\mathrm{G}$ and later type stars), the low collision rate causes the 2 s population to be greater than what is predicted by LTE. For main sequence stars, the LTE population of $2 \mathrm{~s}$ is proportional to $\exp (-E / k T)$, which decreases dramatically when the temperature drops. For luminous giants, our results suggest a shallower dependency of the 2 s population to temperature than in main sequence stars. In the atmospheres of luminous stars, as compared with main sequence stars, fewer hydrogen atoms are piled up in the $n=2$ levels in the high temperature regime while more hydrogen atoms are found in the same levels in the low temperature regime. The difference disappears around the spectral type of F5.

Przybilla \& Butler (2004) point out that purely NLTE effects in the photosphere of Arcturus are not enough to make the model match the observation, and one must consider the NLTE contribution in an extended atmosphere model including the chromosphere. Generally, the inverted temperature structure of the chromosphere (increasing temperature outward) would lead to emission features. However, Mihalas \& Athay (1973) show that strong NLTE effects in the chromosphere can maintain the absorption in the $\mathrm{H} \alpha$ region.

\section{Conclusion}

We have observed a wide range of stars at the $10049 \AA \mathrm{P} \delta$ line and a few stars at the $10938 \AA \mathrm{P} \gamma$ line to make their line profiles available for comparison with model stellar atmospheres. Since the Paschen lines are less affected by the departures from LTE than the Balmer lines, they should be more convenient to use as a test of models. As expected, the difference in line profiles of Paschen lines as compared to $\mathrm{H} \alpha$ is greatest for stars of high luminosity and low effective temperature. 
Acknowledgements. We thank David Bohlender, Les Saddlemyer, Dmitry Monin, Marilyn Bell and other DAO staff for their assistance in making these observations successful. We are also very grateful for the financial support of the Kenilworth Fund of the New York Community Trust. This research has made use of the SIMBAD database, operated at CDS, Strasbourg, France.

\section{References}

Albayrak, B. 2000, A\&A, 364, 237

Asplund, M. 2005, ARA\&A, 43, 481

Bakos, G. A. 1971, JRASC, 65, 222

Barzdis, A. 2010, MNRAS, 408, 1452

Bergemann, M., \& Gehren, T. 2008, A\&A, 492, 823

Burris, D. L., Pilachowski, C. A., Armandroff, T. E., et al. 2000, ApJ, 544, 302

Carr, J. S., Sellgren, K., \& Balachandran, S. C. 2000, ApJ, 530, 307

Cowley, C. R., \& Castelli, F. 2002, A\&A, 387, 595

Erspamer, D., \& North, P. 2003, A\&A, 398, 1121

Fernandez-Villacanas, J. L., Rego, M., \& Cornide, M. 1990, AJ, 99, 1961

Fuhrmann, K. 2008, MNRAS, 384, 173

Fulbright, J. P. 2000, AJ, 120, 1841

Gies, D. R., \& Lambert, D. 1992, ApJ, 387, 673

Gonzalez, G., Carlson, M. K., \& Tobin, R. W. 2010, MNRAS, 403, 1368

Hekker, S., \& Meléndez, J. 2007, A\&A, 475, 1003

Hill, G. M. 1995, A\&A, 294, 536

Hui-Bon-Hoa, A. 2000, A\&AS, 144, 203

Ito, H., Aoki, W., Honda, S., \& Beers, T. C. 2009, ApJ, 698, L371
Kurucz, R. L. 1993a, ATLAS9 Stellar Atmosphere Programs and $2 \mathrm{~km} \mathrm{~s}^{-1}$ grid, Kurucz CD-ROM No. 13 (Cambridge, Mass.: Smithsonian Astrophysical Observatory)

Luck, R. E., \& Bond, H. E. 1980, ApJ, 241, 218

Luck, R. E., \& Lambert, D. L. 1981, ApJ, 245, 1018

Lyubimkov, L. S., Lambert, D. L., Rostopchin, S. I., Rachkovskaya, T. M., \& Poklad, D. B. 2010, MNRAS, 402, 1369

Mallik, S. V. 1998, A\&A, 338, 623

McWilliam, A. 1990, ApJS, 74, 1075

Meléndez, J., Asplund, M., Alves-Brito, A., et al. 2008, A\&A, 484, 21

Mihalas, D., \& Athay, R. G. 1973, ARA\&A, 11, 187

Mishenina, T. V., \& Kovtyukh, V. V. 2001, A\&A, 370, 951

Peters, G. J., \& Aller, L. H. 1970, ApJ, 159, 525

Przybilla, N., \& Butler, K. 2004, ApJ, 610, 61

Sbordone, L. 2005, Mem. Soc. Astron. Ital. Suppl., 8, 61

Sbordone, L., Bonifacio, P., Castelli, F., \& Kurucz, R. L. 2004, Mem. Soc. Astron. Ital. Suppl., 5, 93

Schuster, A. 1902, ApJ, 16, 320

Schuster, A. 1905, ApJ, 21, 1

Schwarzchild, K. 1906, Goettinger Nachr., 41

Smith, K. C., \& Dworetsky, M. M. 1993, A\&A, 274, 335

Smith, V. V., \& Lambert, D. L. 1985, ApJ, 294, 326

Spitzer, L. S. 1939, ApJ, 90, 494

Struve, O., Wurm, K., \& Henyey, L. G. 1939, PNAS, 25, 67

Unsöld, A., \& Weidemann, V. 1955, VA, 1, 249

van Leeuwen, F. 2007, A\&A, 474, 653

Venn, K. A. 1995, ApJS, 99, 659

Vidal, C. R., Cooper, J., \& Smith, E. W. 1973, ApJS, 25, 37 
W. Huang et al.: A catalogue of Paschen-line profiles
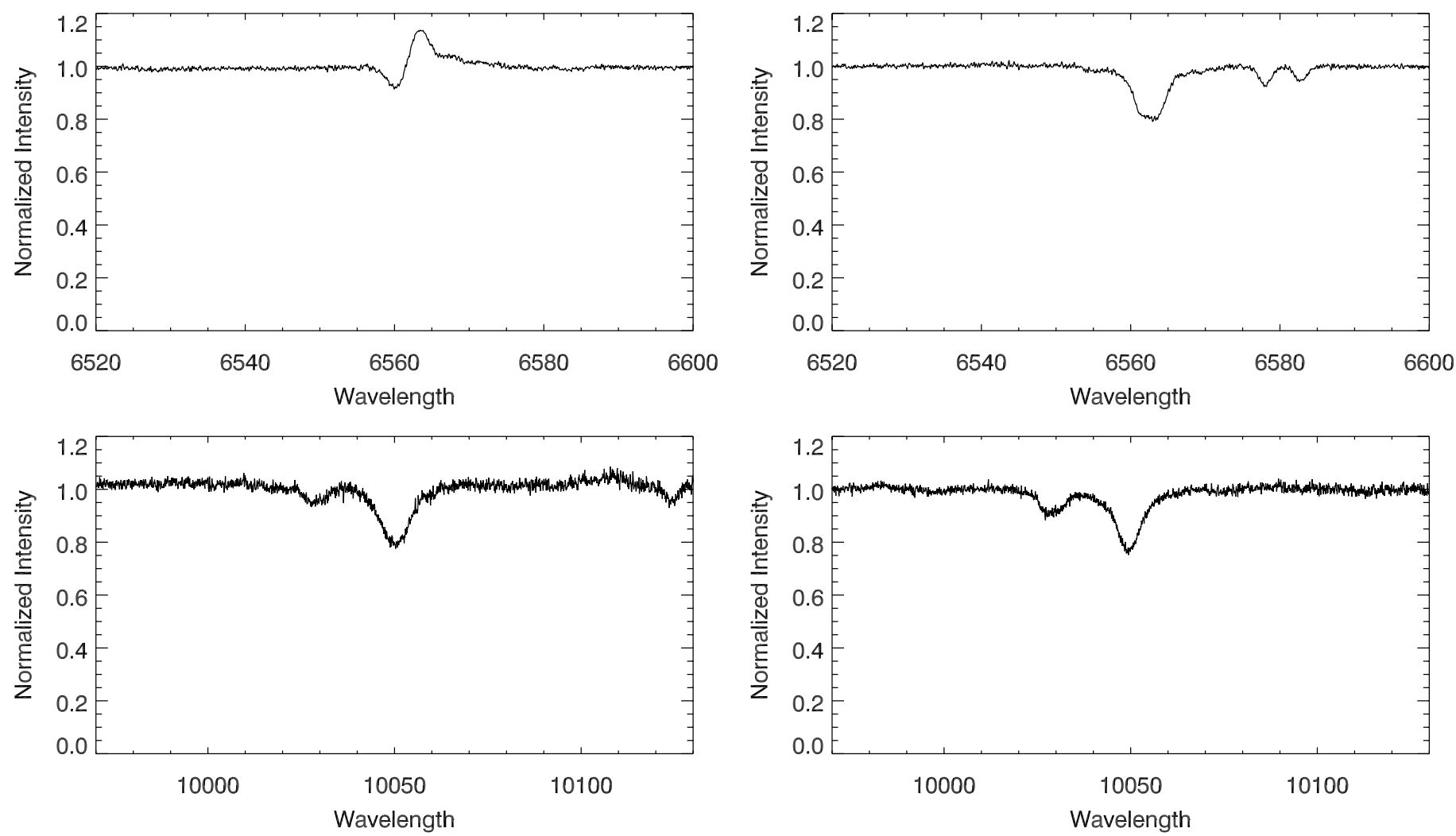

Fig. 7. Profiles of $\mathrm{H} \alpha$ and $\mathrm{P} \delta$ in $\mathrm{HD} 37128$, B0 Iab.

Fig. 9. Profiles of $\mathrm{H} \alpha$ and $\mathrm{P} \delta$ in HD 91316, B1 Iab.
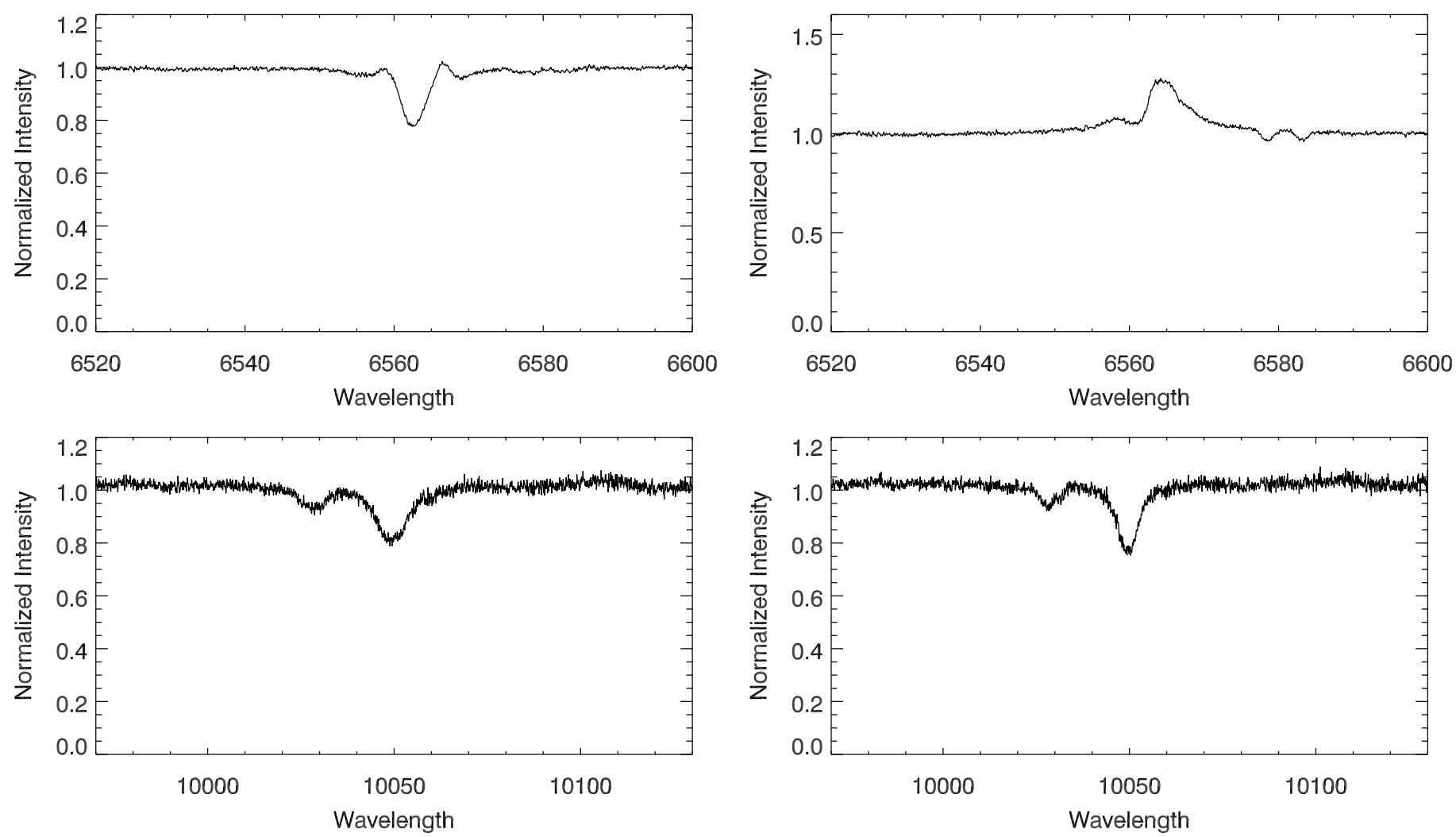

Fig. 8. Profiles of $\mathrm{H} \alpha$ and $\mathrm{P} \delta$ in $\mathrm{HD}$ 40111, B0.5 II.

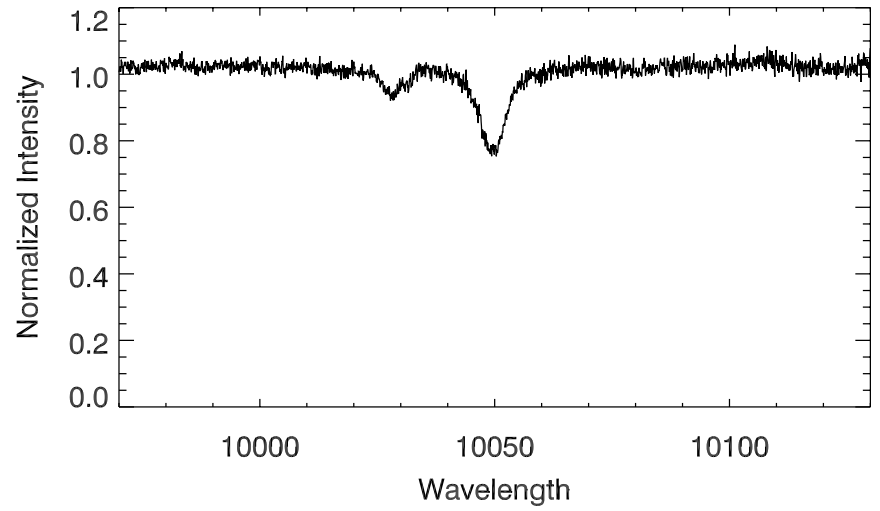

Fig. 10. Profiles of $\mathrm{H} \alpha$ and $\mathrm{P} \delta$ in $\mathrm{HD} 2905$, B 1 Iae. 
A\&A 547, A62 (2012)
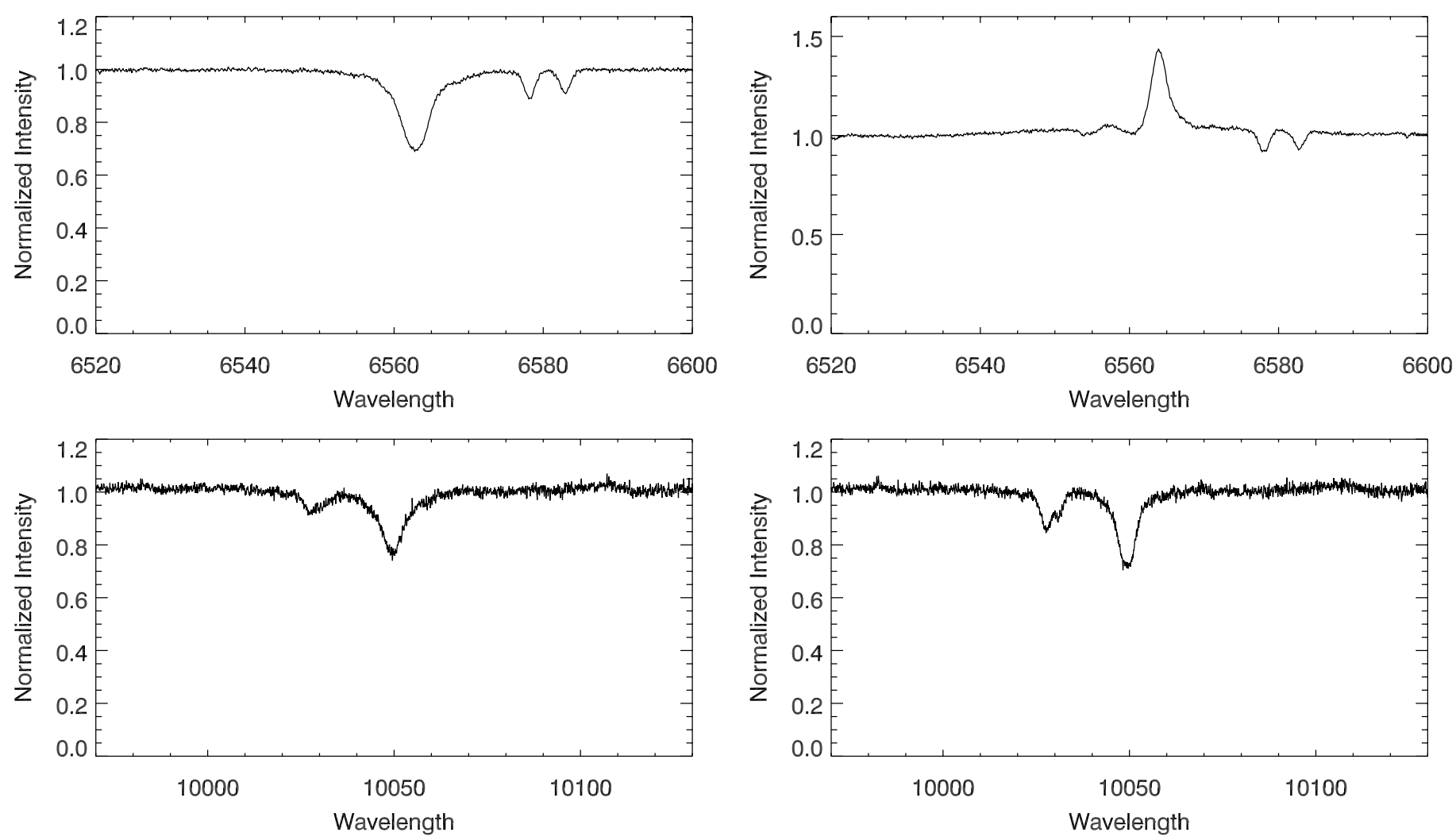

Fig. 11. Profiles of $\mathrm{H} \alpha$ and $\mathrm{P} \delta$ in D24398, B1 Ib.

Fig. 13. Profiles of $\mathrm{H} \alpha$ and $\mathrm{P} \delta$ in HD 41117, B2 Iaev.
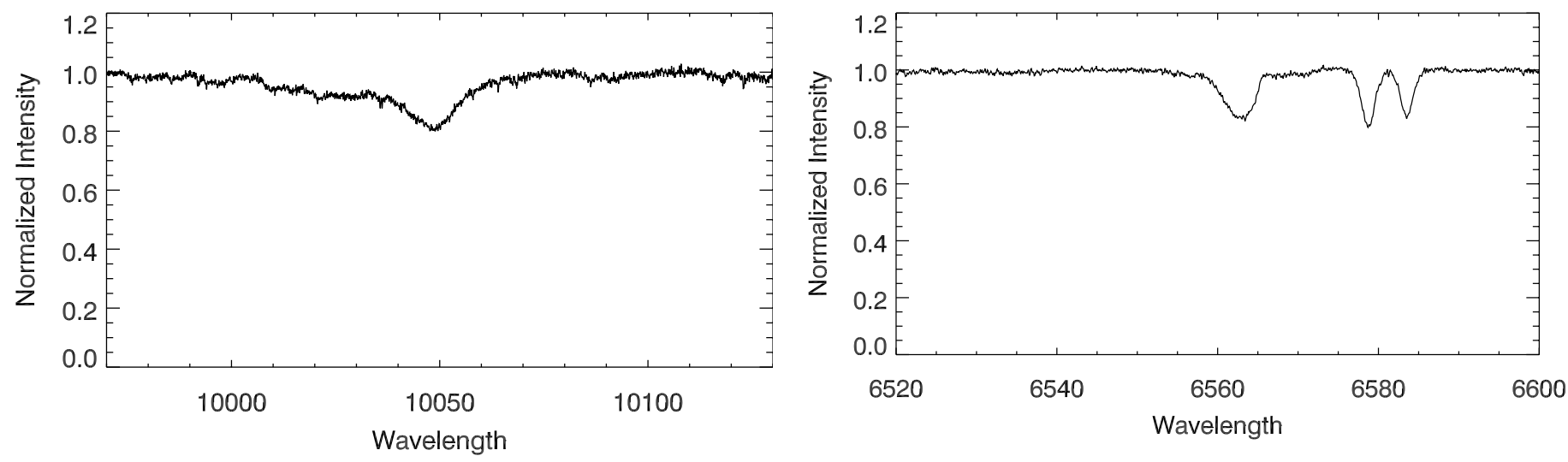

Fig. 12. Profile of $\mathrm{P} \delta$ in HD 116658, B1 III.

Fig. 14. Profile of $\mathrm{H} \alpha$ in HD 206165, B2 Ib. 
W. Huang et al.: A catalogue of Paschen-line profiles
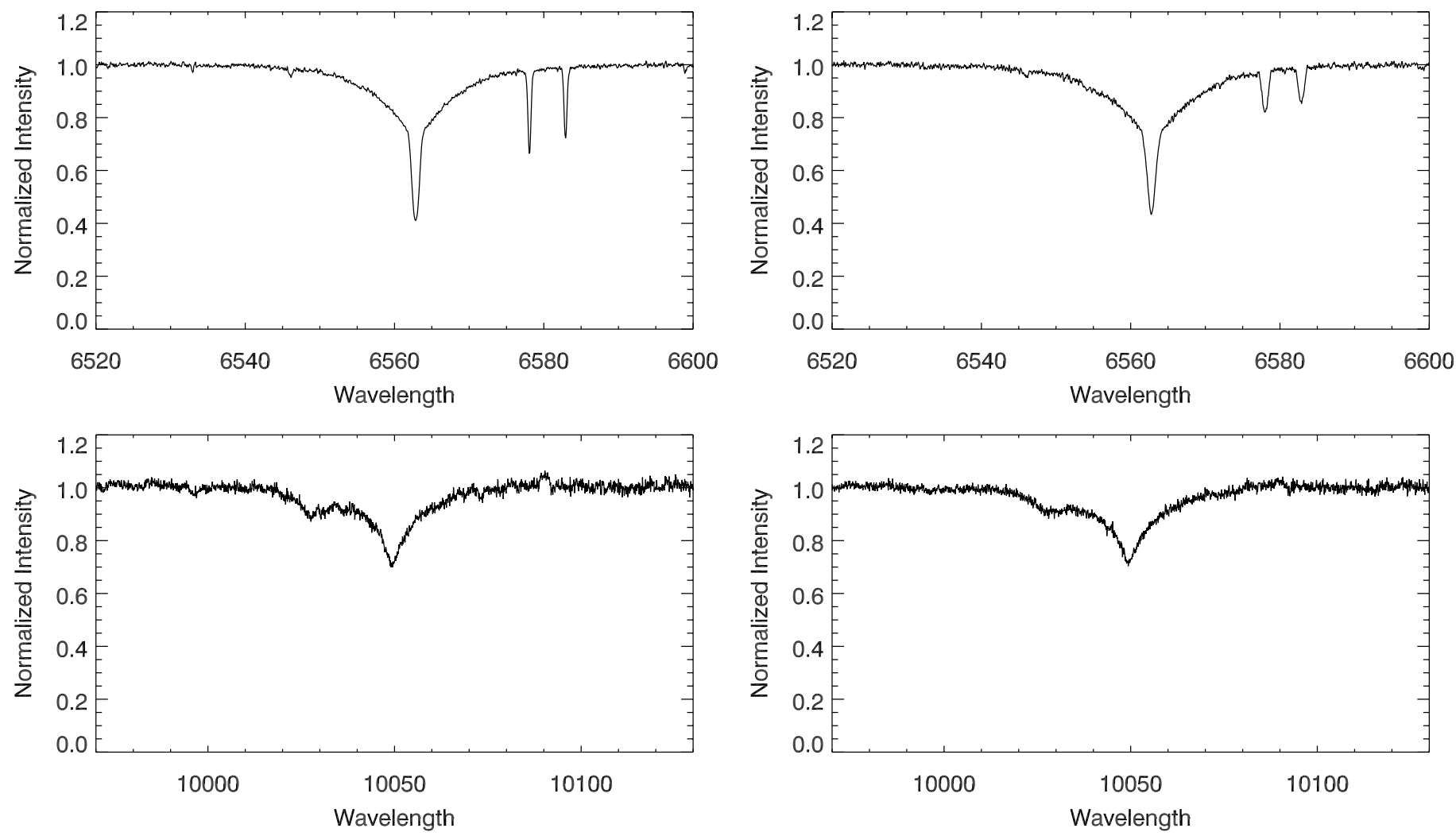

Fig. 15. Profiles of $\mathrm{H} \alpha$ and $\mathrm{P} \delta$ in $\mathrm{HD} 886, \mathrm{~B} 2$ IV.

Fig. 17. Profiles of $\mathrm{H} \alpha$ and $\mathrm{P} \delta$ in $\mathrm{HD} 35708, \mathrm{~B} 2.5 \mathrm{IV}$.
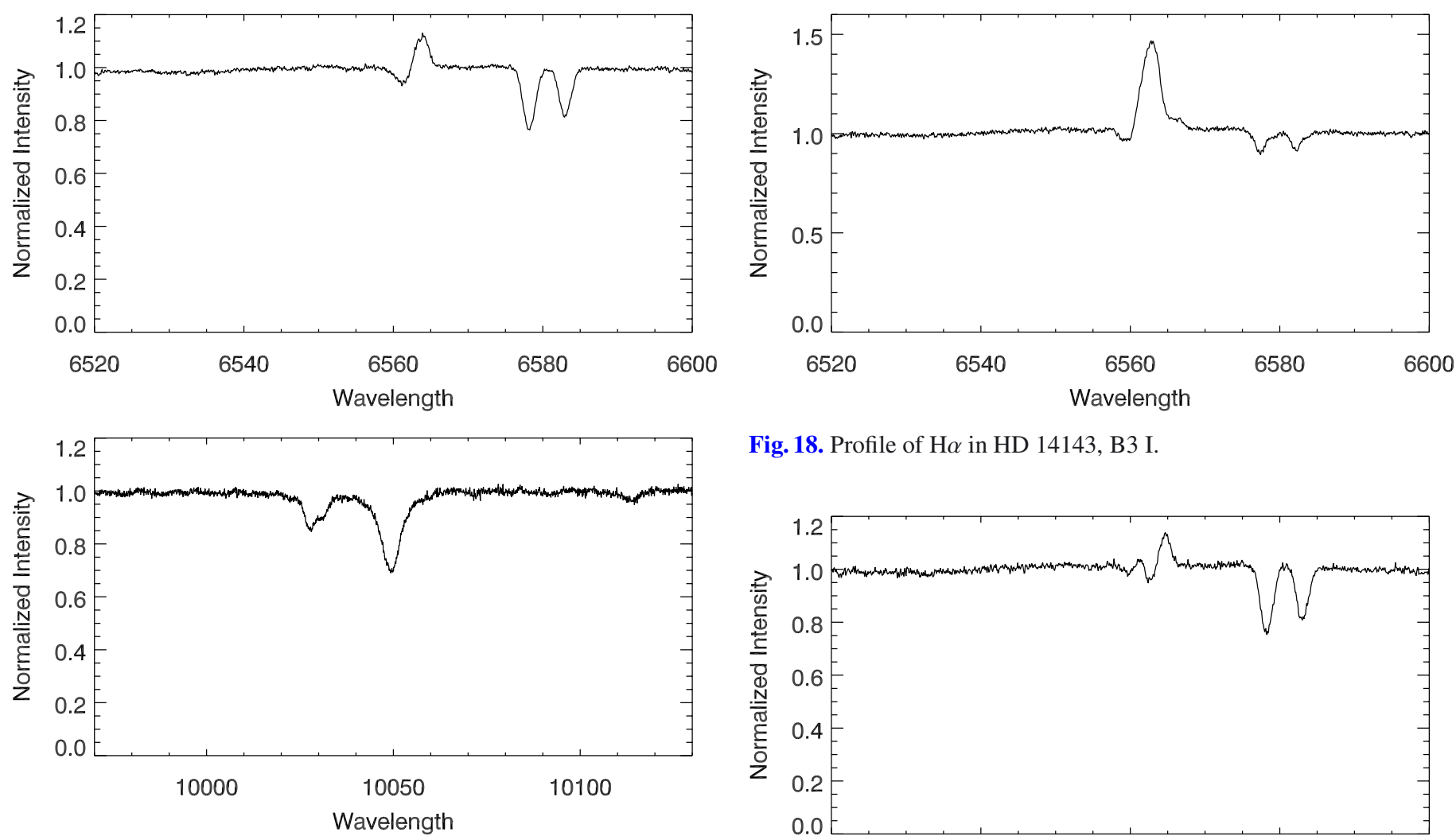

Fig. 16. Profiles of $\mathrm{H} \alpha$ and $\mathrm{P} \delta$ in $\mathrm{HD} 198478$, B2.5 Ia.

Fig. 18. Profile of $\mathrm{H} \alpha$ in HD 14143, B3 I.

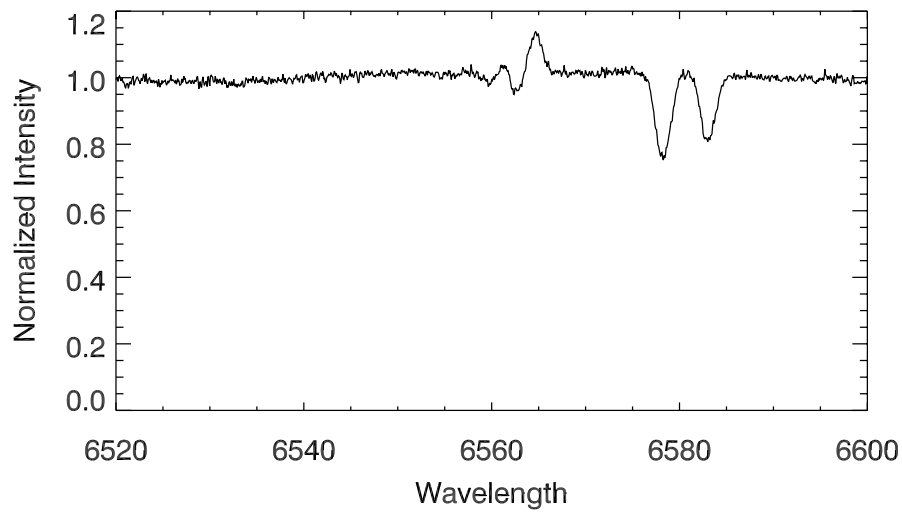

Fig. 19. Profile of $\mathrm{H} \alpha$ in HD 14134, B3 Ia. 
A\&A 547, A62 (2012)
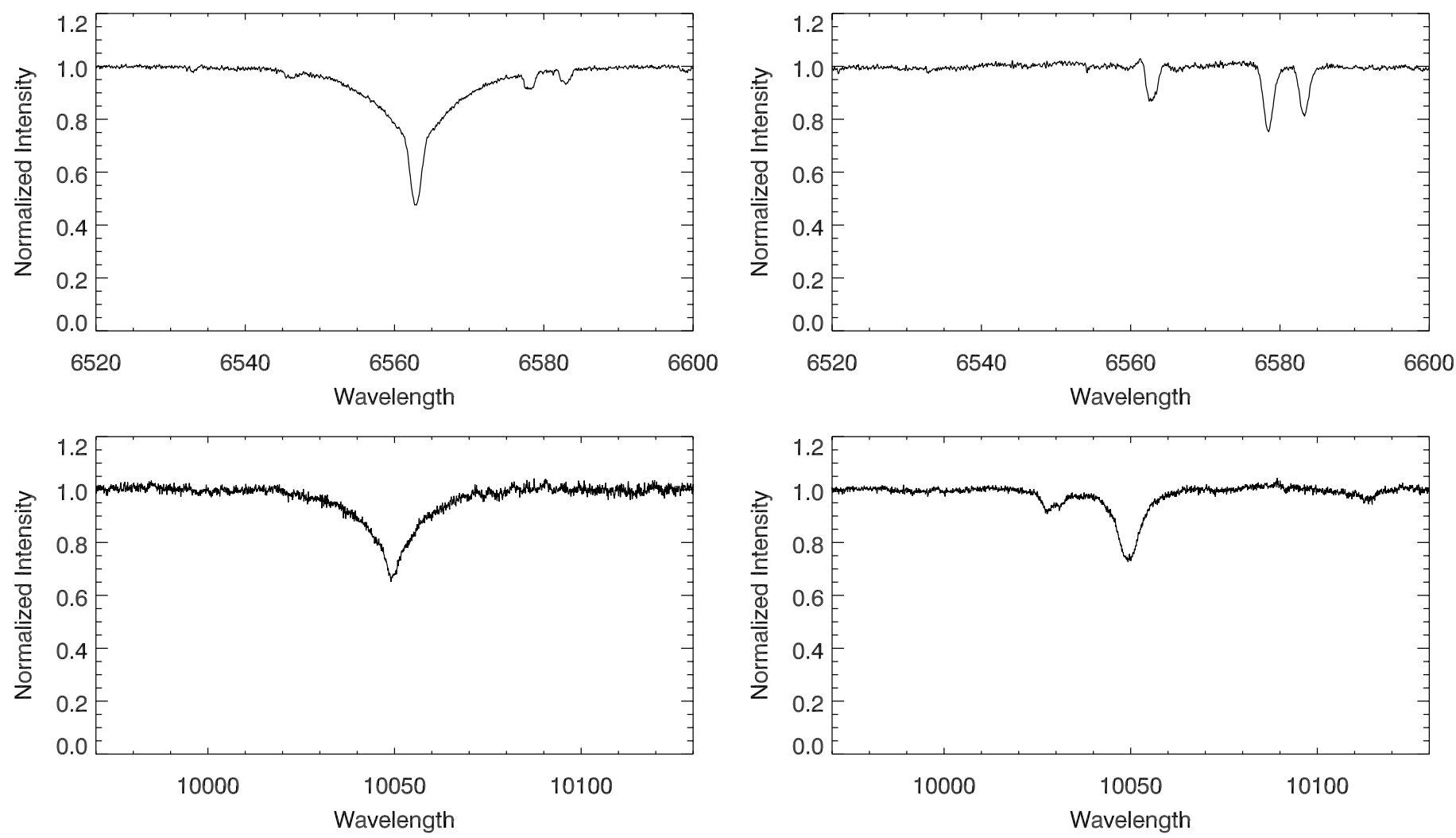

Fig. 20. Profiles of $\mathrm{H} \alpha$ and $\mathrm{P} \delta$ in HD 11415, B3 III.
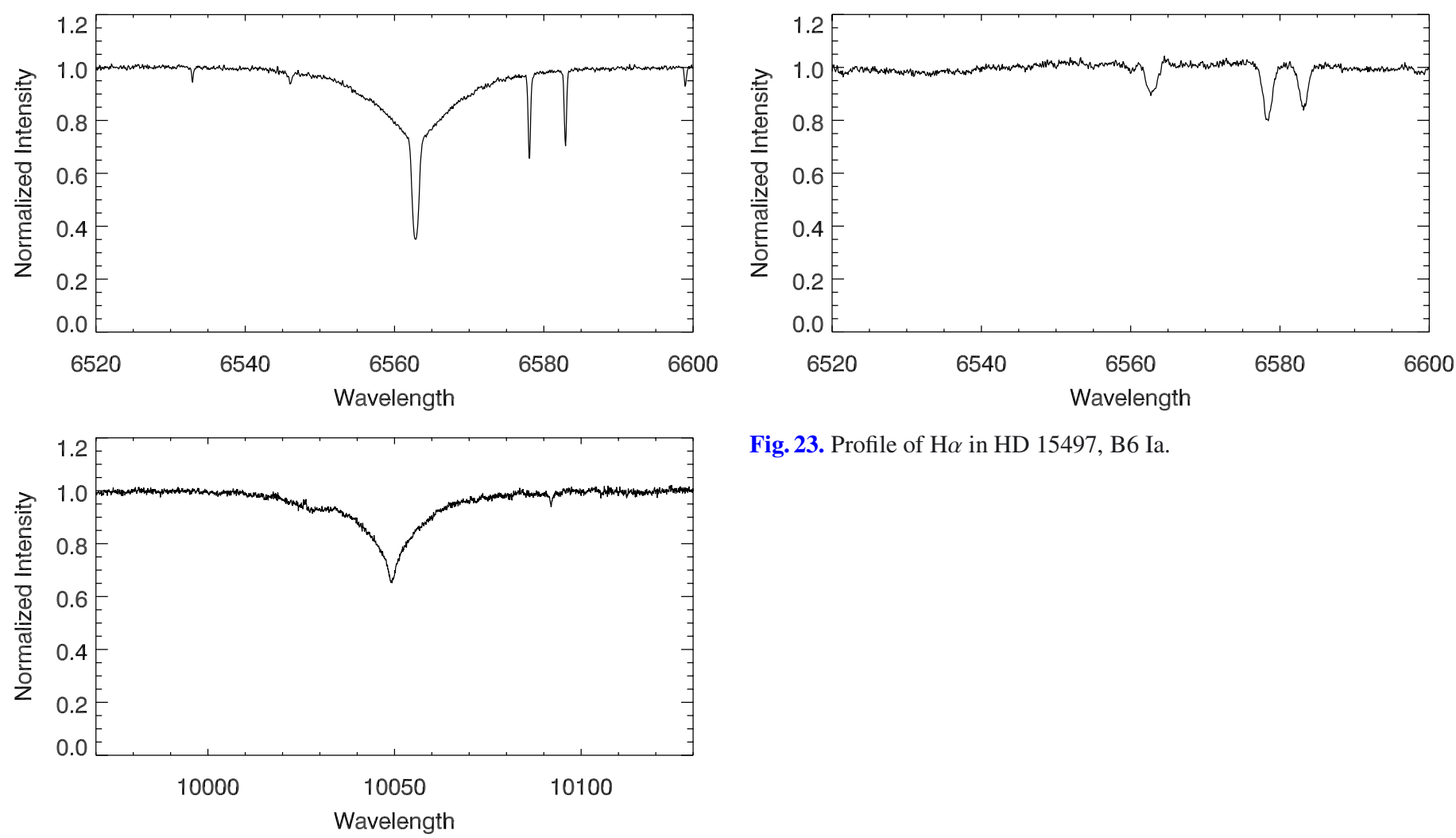

Fig. 23. Profile of $\mathrm{H} \alpha$ in HD 15497, B6 Ia.

Fig. 21. Profiles of $\mathrm{H} \alpha$ and $\mathrm{P} \delta$ in HD 160762, B3 IV. 
W. Huang et al.: A catalogue of Paschen-line profiles
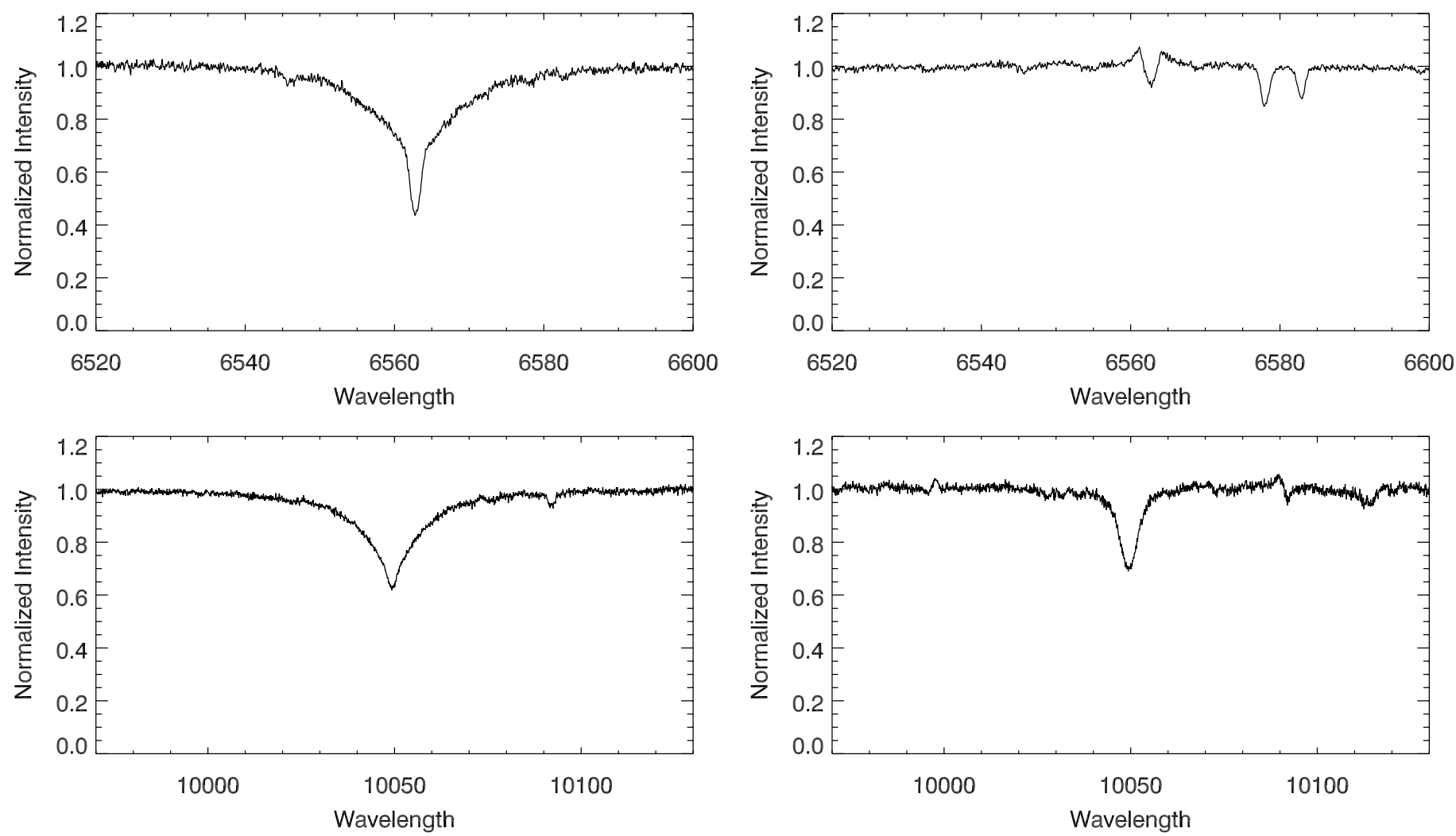

Fig. 24. Profiles of $\mathrm{H} \alpha$ and $\mathrm{P} \delta$ in HD 155763, B6 III.

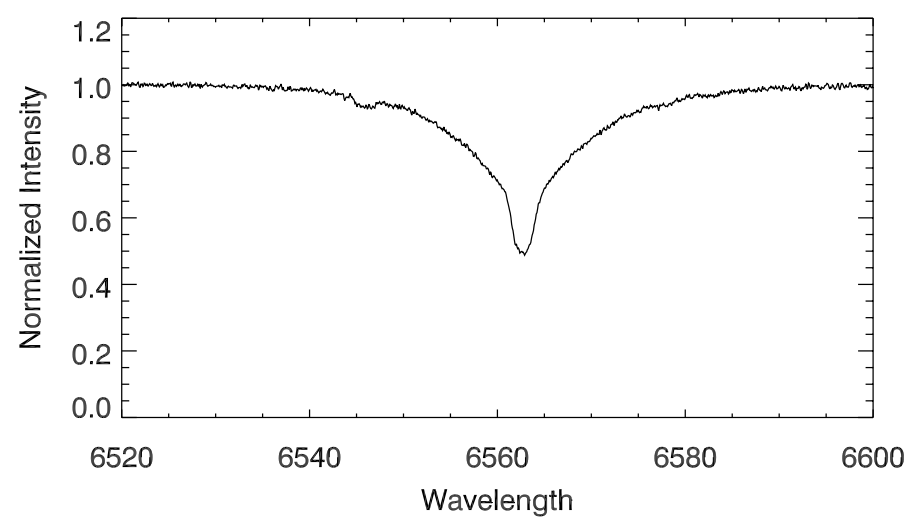

Fig. 25. Profile of H $\alpha$ in HD 3240, B7 III.

Fig. 27. Profiles of $\mathrm{H} \alpha$ and $\mathrm{P} \delta$ in $\mathrm{HD} 34085$, B8 Iab.

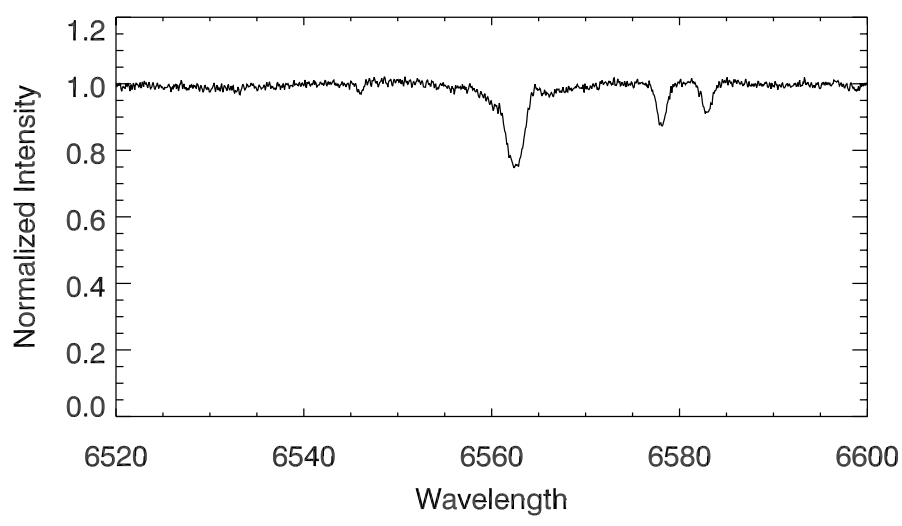

Fig. 28. Profile of $\mathrm{H} \alpha$ in HD 14322, B8 Ib.

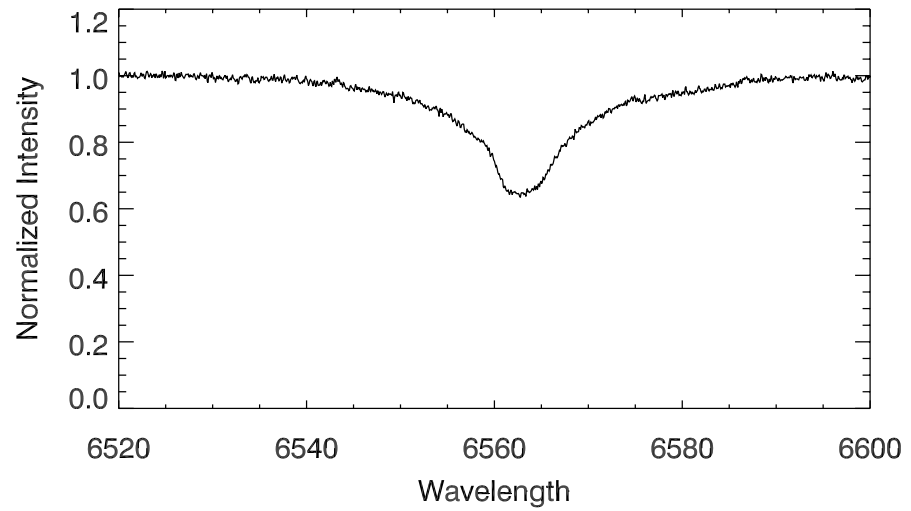

Fig. 26. Profile of $\mathrm{H} \alpha$ in $\mathrm{HD} 69686, \mathrm{~B} 8$. 
A\&A 547, A62 (2012)
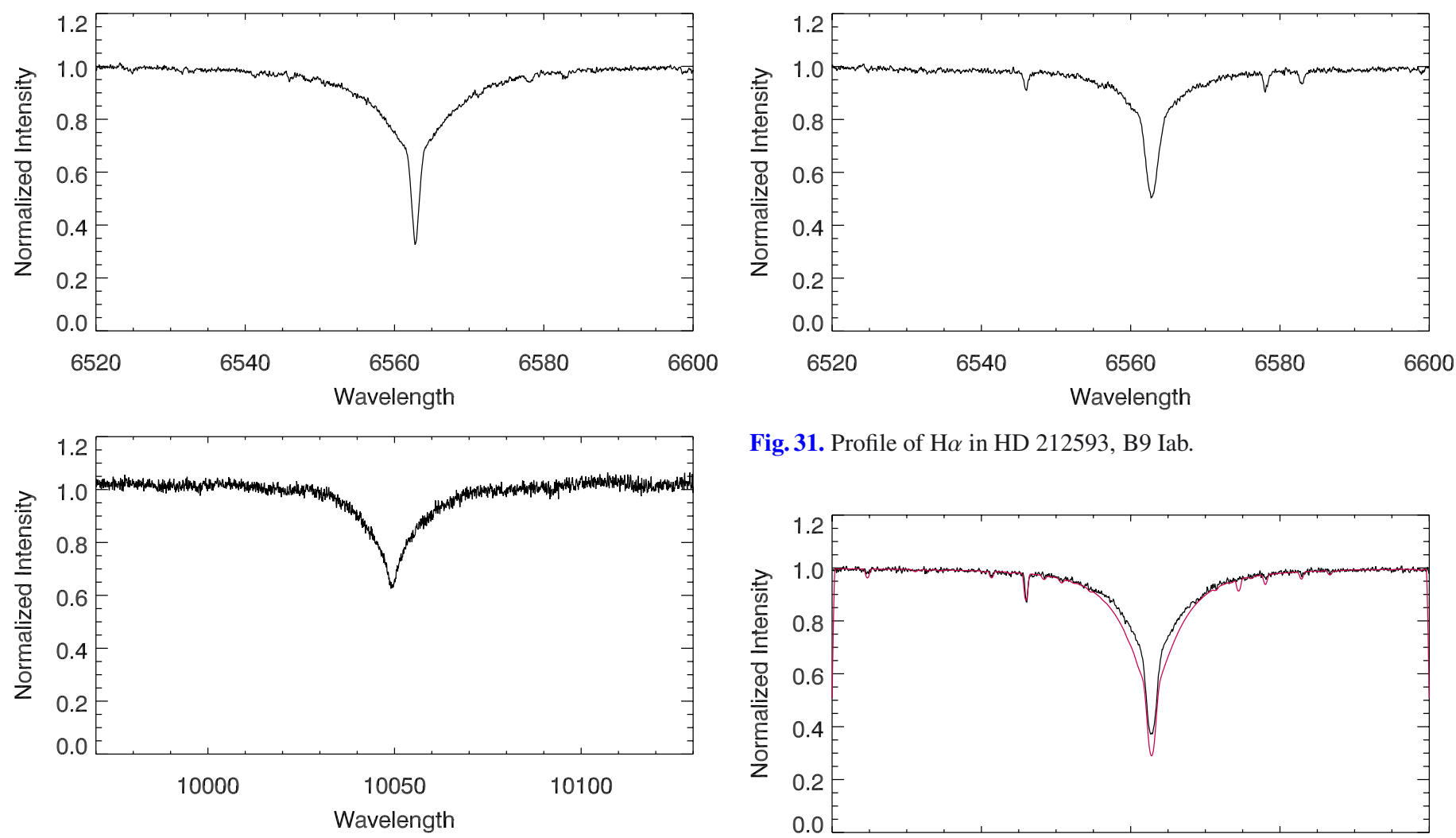

Fig. 31. Profile of $\mathrm{H} \alpha$ in HD 212593, B9 Iab.
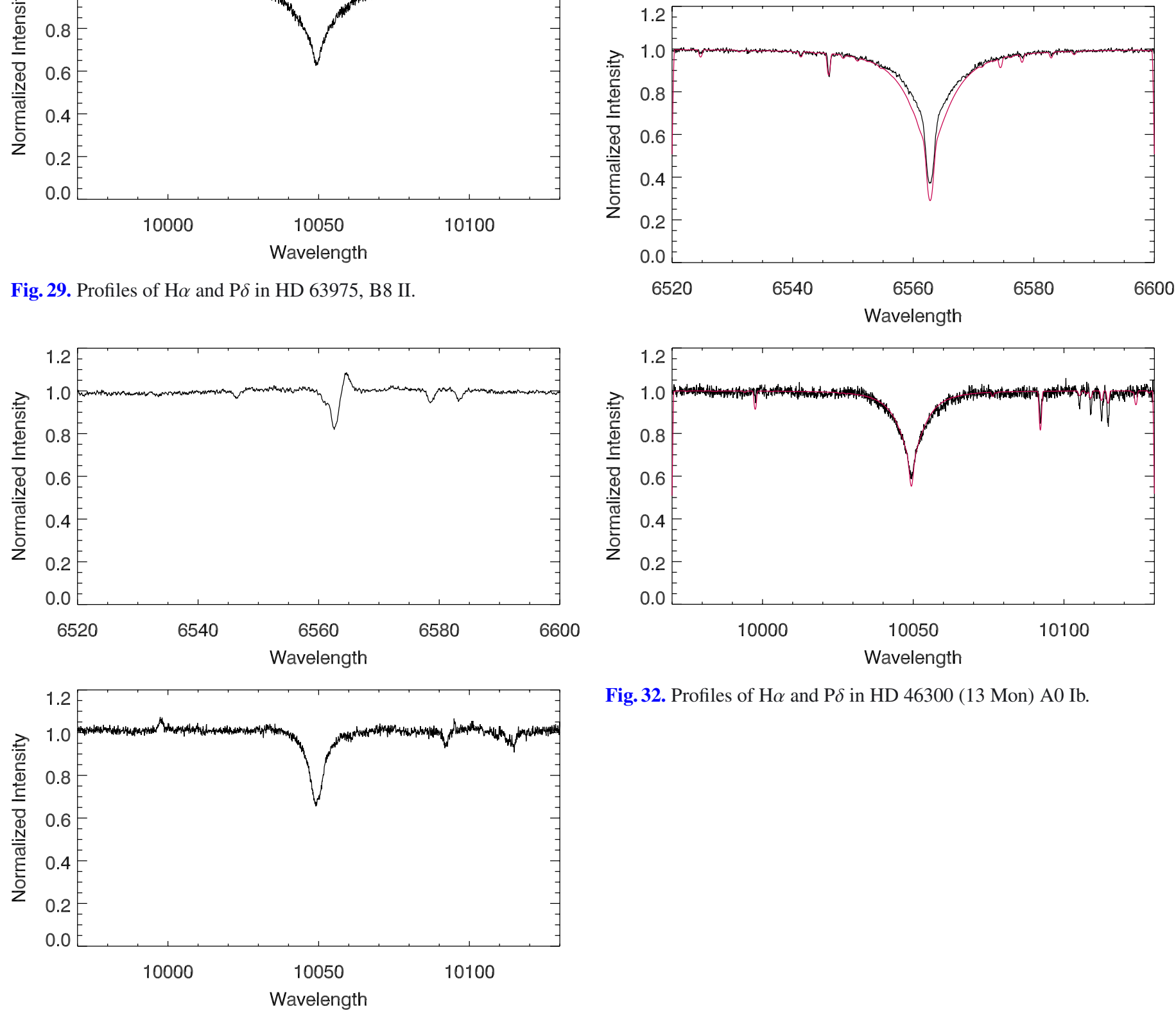

Fig. 32. Profiles of $\mathrm{H} \alpha$ and $\mathrm{P} \delta$ in $\mathrm{HD} 46300$ (13 Mon) A0 Ib.

Fig. 30. Profiles of $\mathrm{H} \alpha$ and $\mathrm{P} \delta$ in $\mathrm{HD} 21291$, B9 Ia. 
W. Huang et al.: A catalogue of Paschen-line profiles
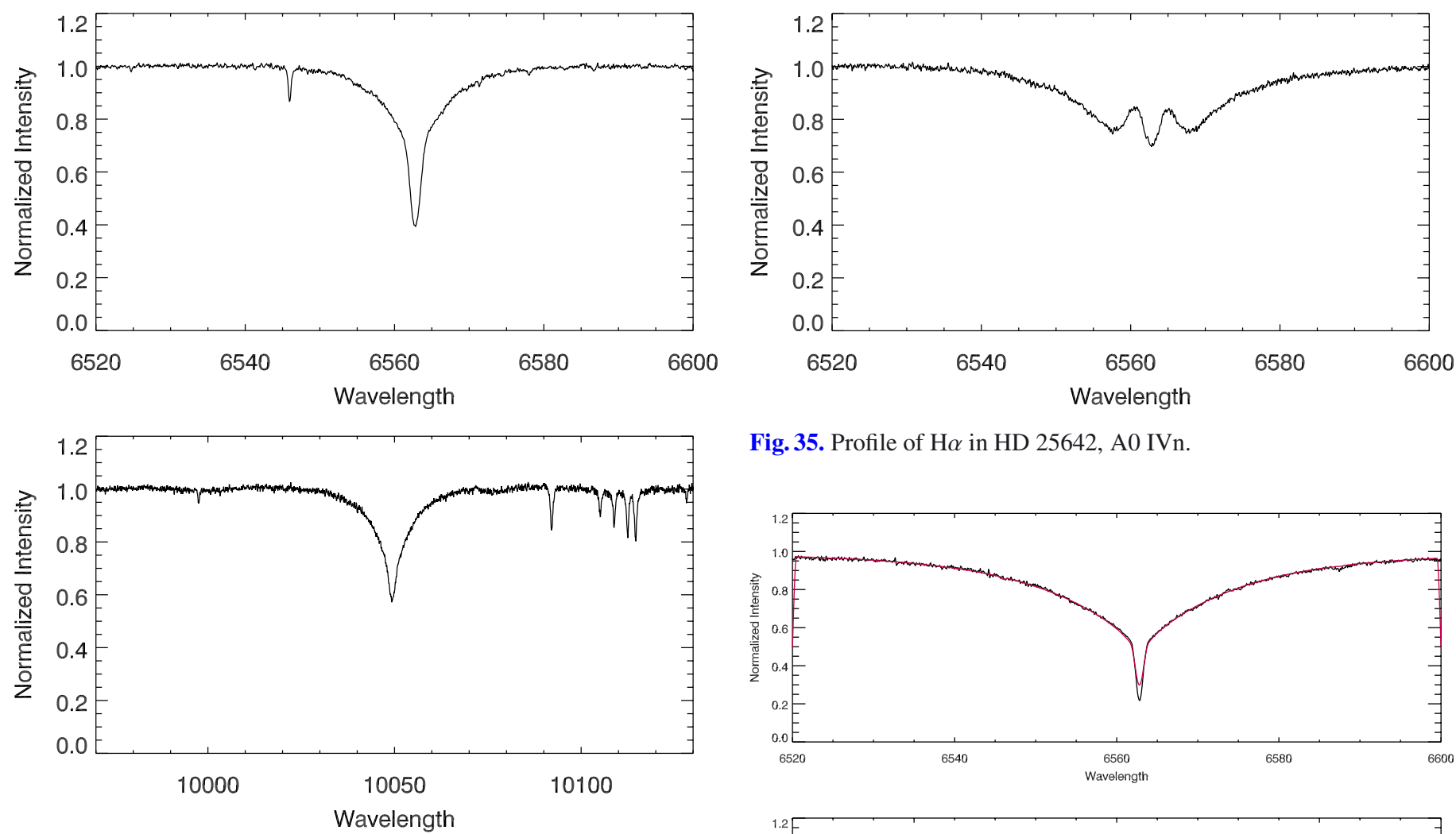

Fig. 35. Profile of $\mathrm{H} \alpha$ in HD 25642, A0 IVn.

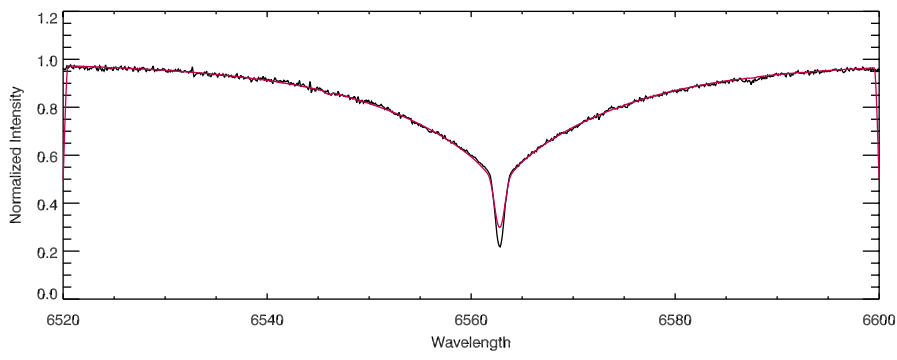

Fig. 33. Profiles of $\mathrm{H} \alpha$ and $\mathrm{P} \delta$ in $\mathrm{HD} 87737$, A0 Ib.
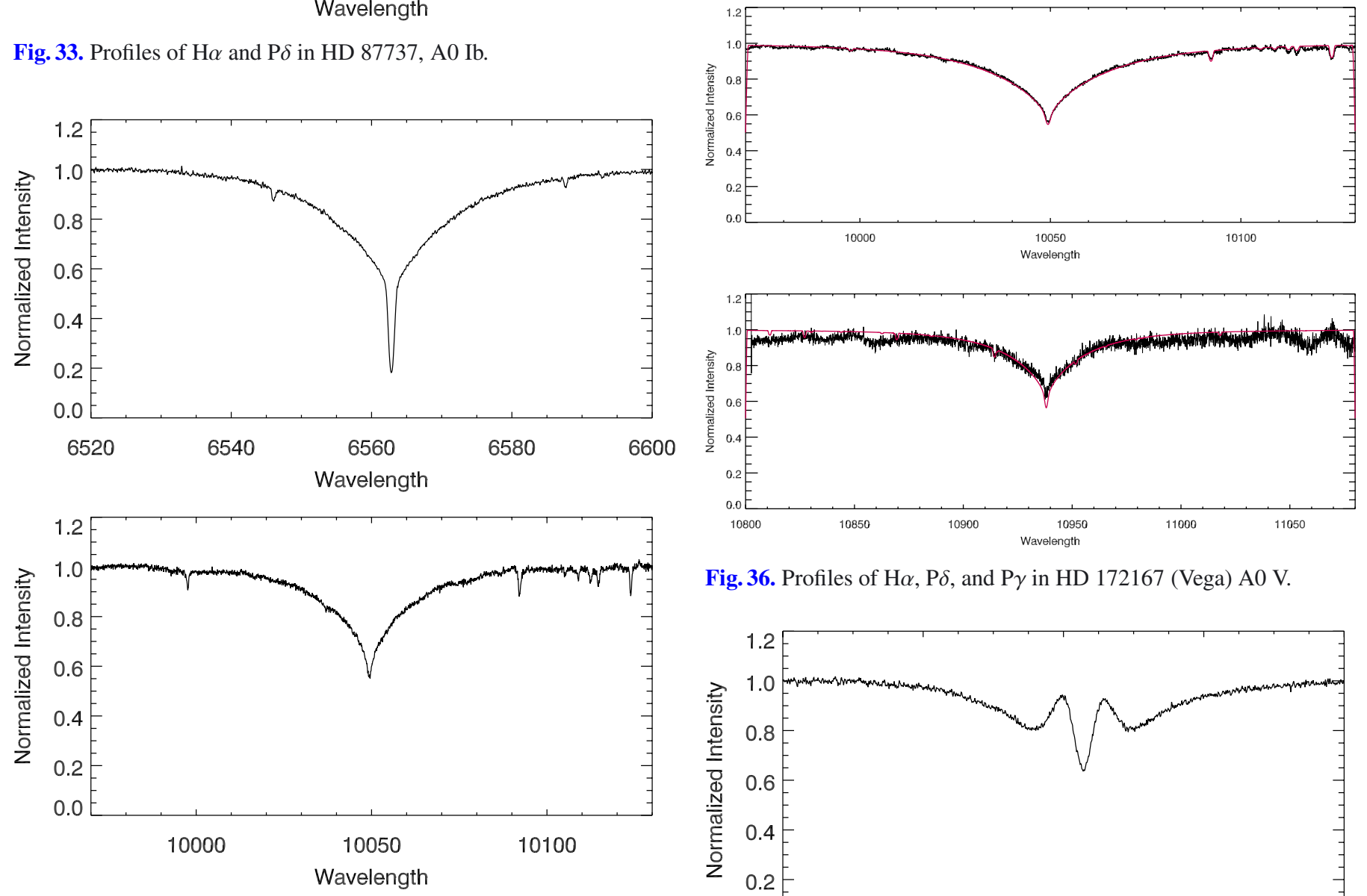

Fig. 34. Profiles of $\mathrm{H} \alpha$ and $\mathrm{P} \delta$ in $\mathrm{HD} 47105$, A0 IV.

Fig. 36. Profiles of $\mathrm{H} \alpha, \mathrm{P} \delta$, and $\mathrm{P} \gamma$ in HD 172167 (Vega) A0 V.

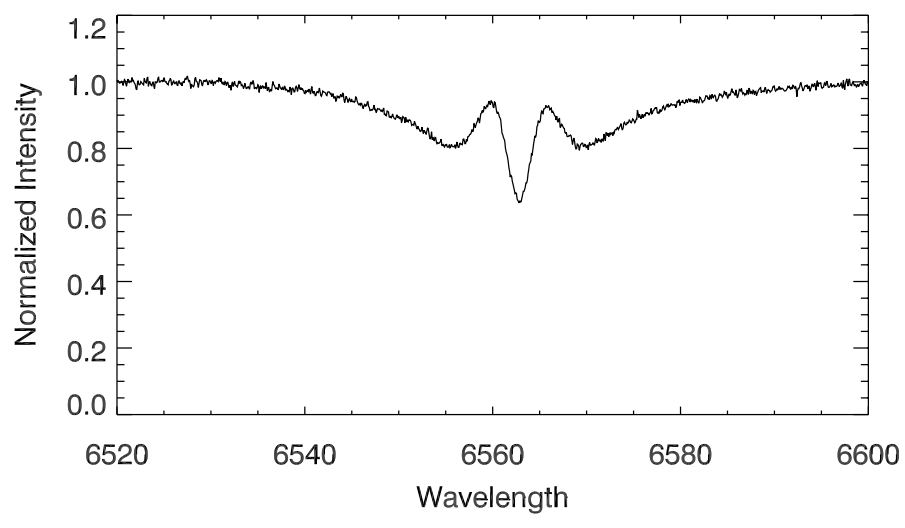

Fig. 37. Profile of $\mathrm{H} \alpha$ in HD 34787, A0 Vne. 
A\&A 547, A62 (2012)
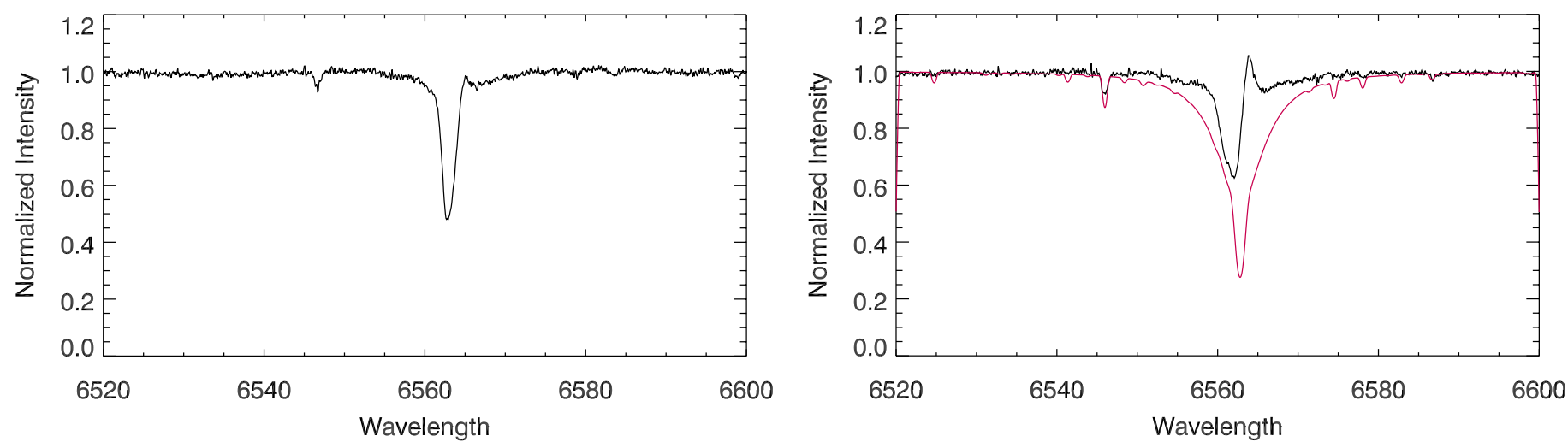

Fig. 38. Profile of $\mathrm{H} \alpha$ in HD 14433, A1 Ia.
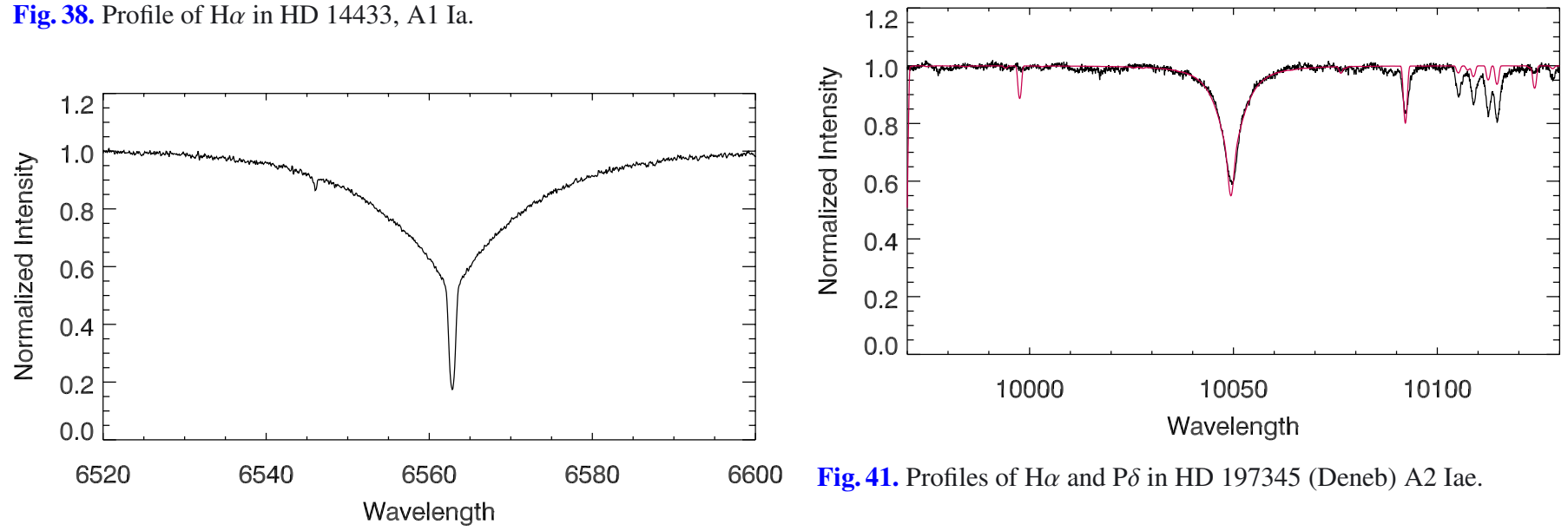

Fig. 41. Profiles of $\mathrm{H} \alpha$ and $\mathrm{P} \delta$ in HD 197345 (Deneb) A2 Iae.

Fig. 39. Profile of $\mathrm{H} \alpha$ in HD 214994, A1 IV.
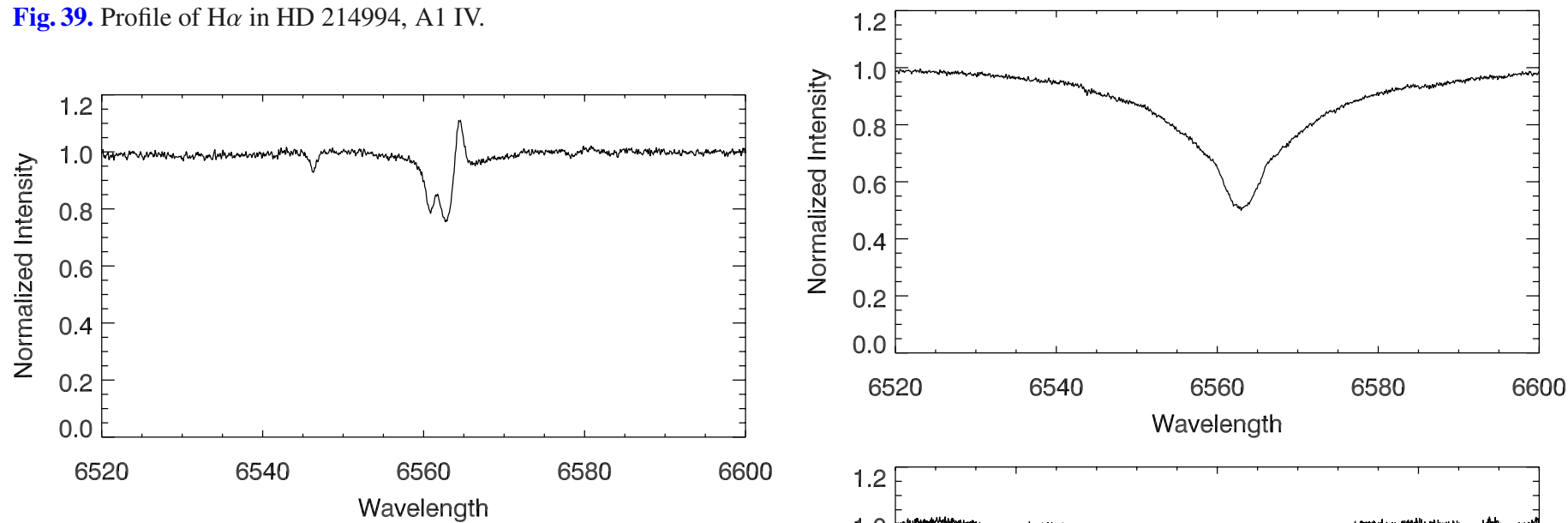

Fig. 40. Profile of $\mathrm{H} \alpha$ in HD 14489, A2 Ia.

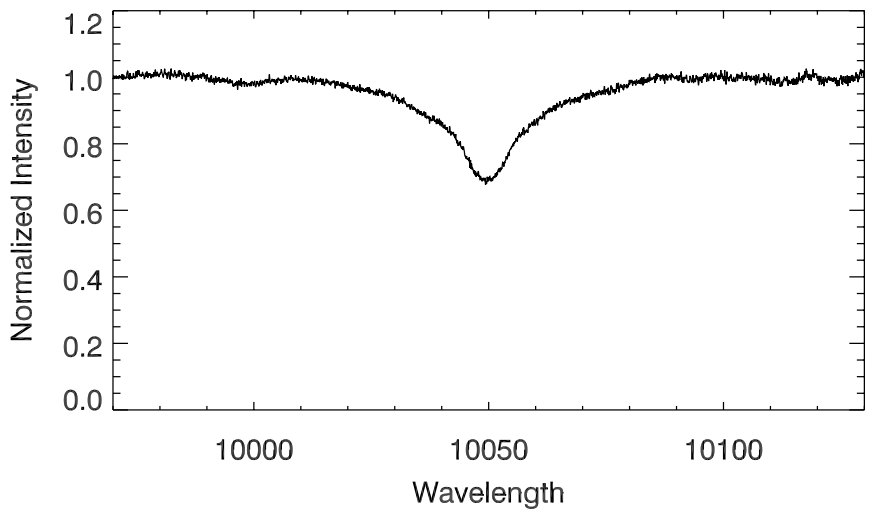

Fig. 42. Profiles of $\mathrm{H} \alpha$ and $\mathrm{P} \delta$ in HD 50019, A3 III. 
W. Huang et al.: A catalogue of Paschen-line profiles
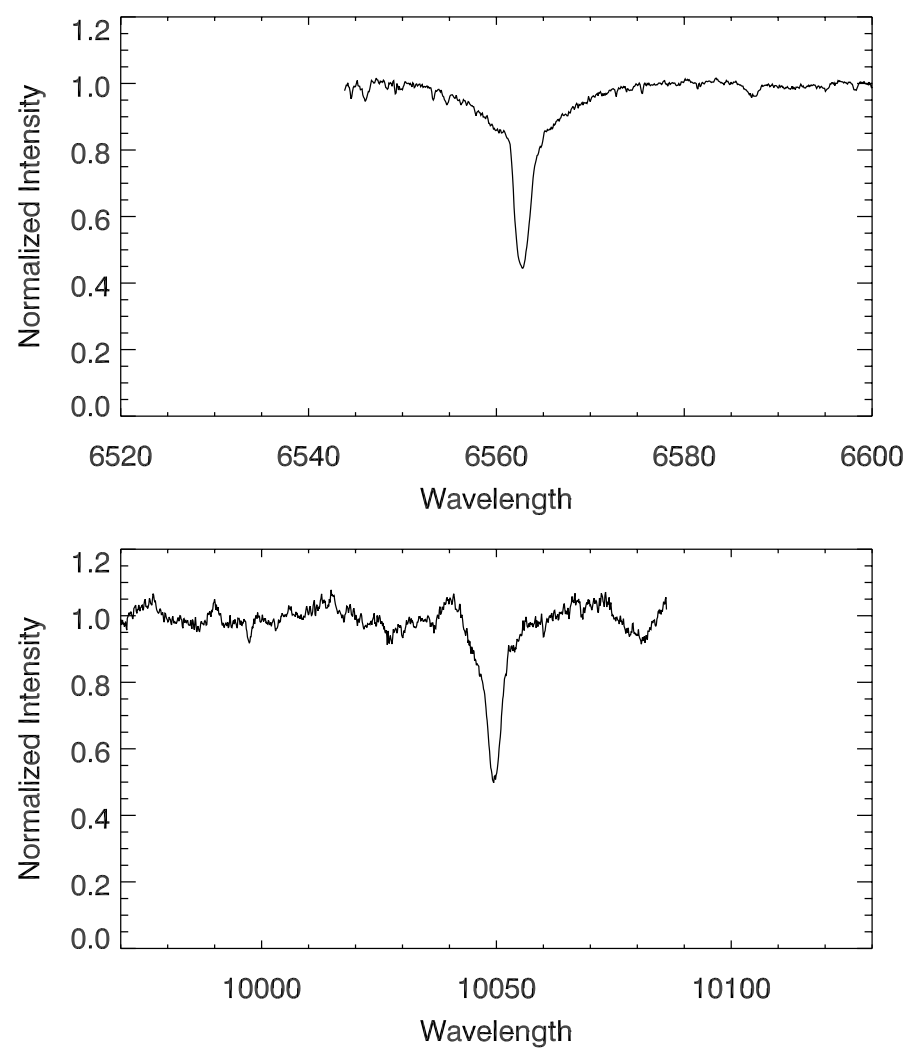

Fig. 43. Profiles of $\mathrm{H} \alpha$ and $\mathrm{P} \delta$ in HD 17378, A5 Ia. The limited wavelength coverage is due to the display of one order of the echelle.

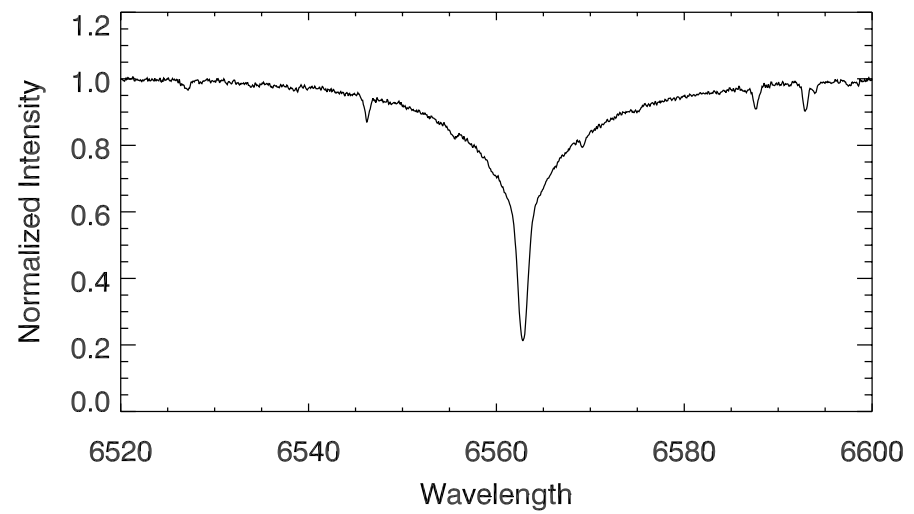

Fig. 44. Profile of $\mathrm{H} \alpha$ in HD 2628, A7 III.
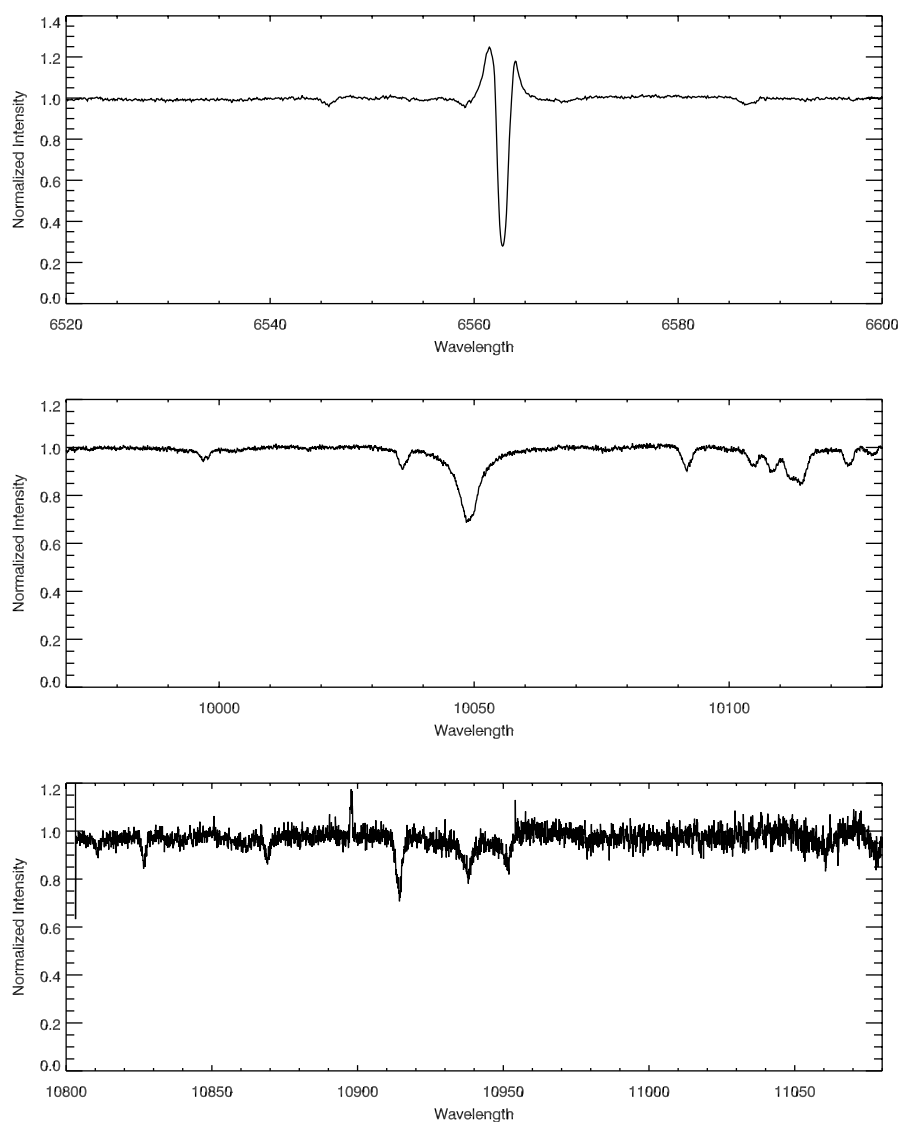

Fig. 45. Profiles of $\mathrm{H} \alpha, \mathrm{P} \delta$, and $\mathrm{P} \gamma$ in HD 31964, A8 Iab.
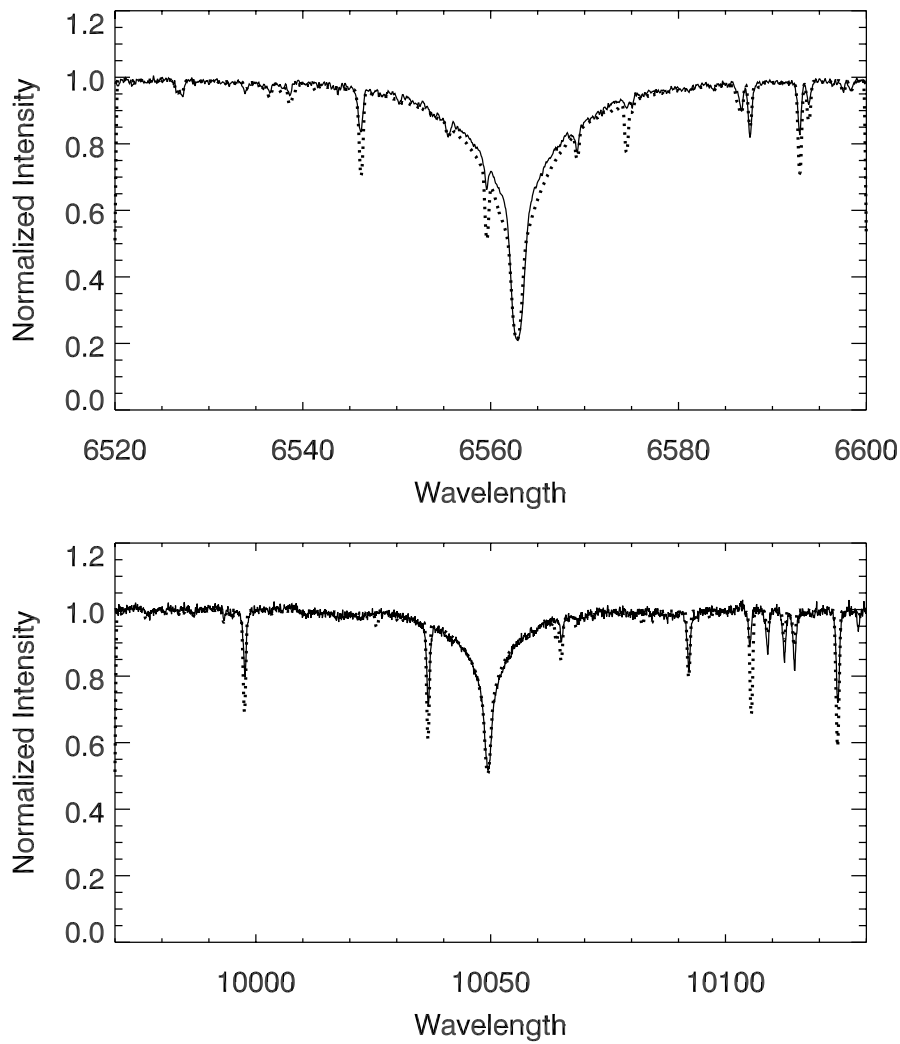

Fig. 46. Profiles of $\mathrm{H} \alpha$ and $\mathrm{P} \delta$ in $\mathrm{HD} 182835$, F2 Iab. 

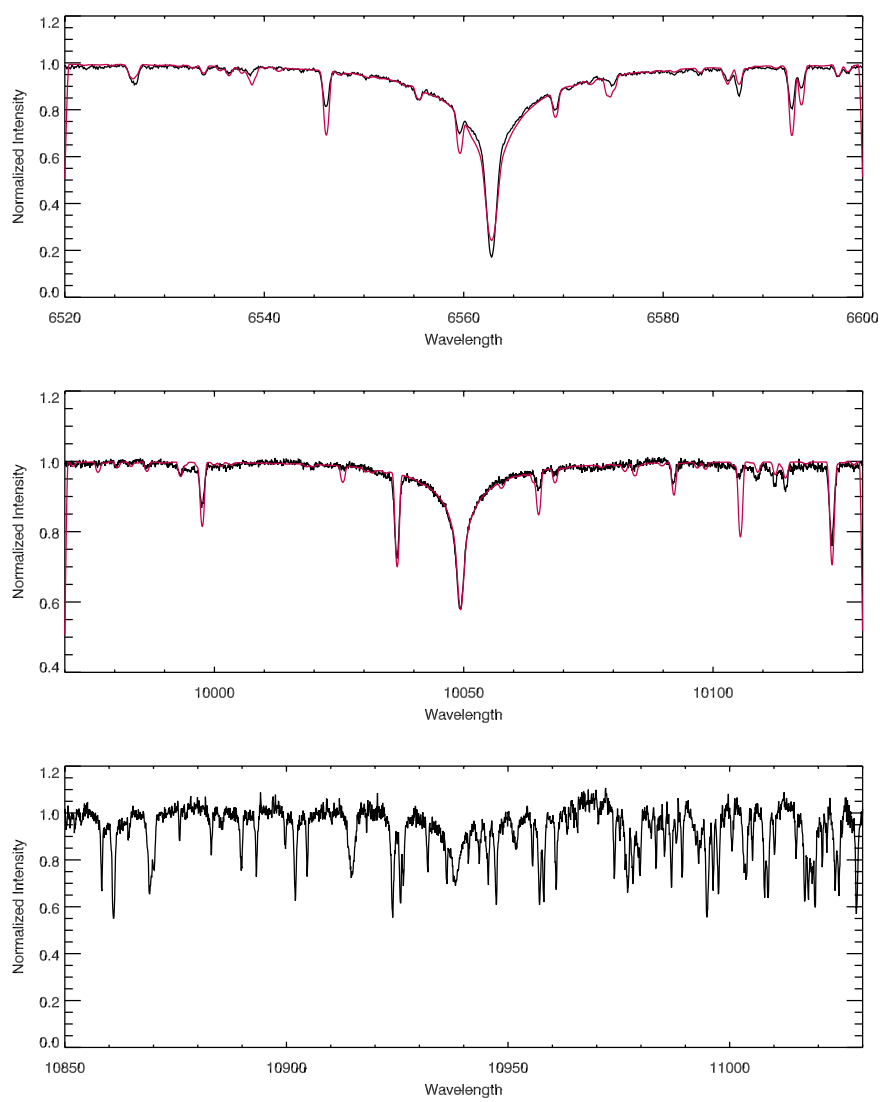

Fig. 47. Profiles of $\mathrm{H} \alpha, \mathrm{P} \delta$, and $\mathrm{P} \gamma$ in HD 20902 ( $\alpha$ Per) F5 Iab.

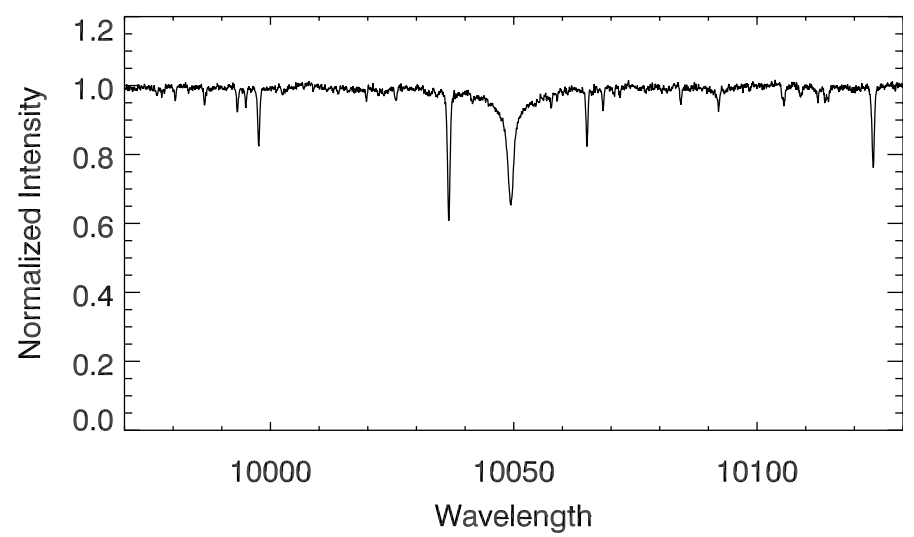

Fig. 48. Profile of $\mathrm{P} \delta$ in HD 213306, F5 Iab.

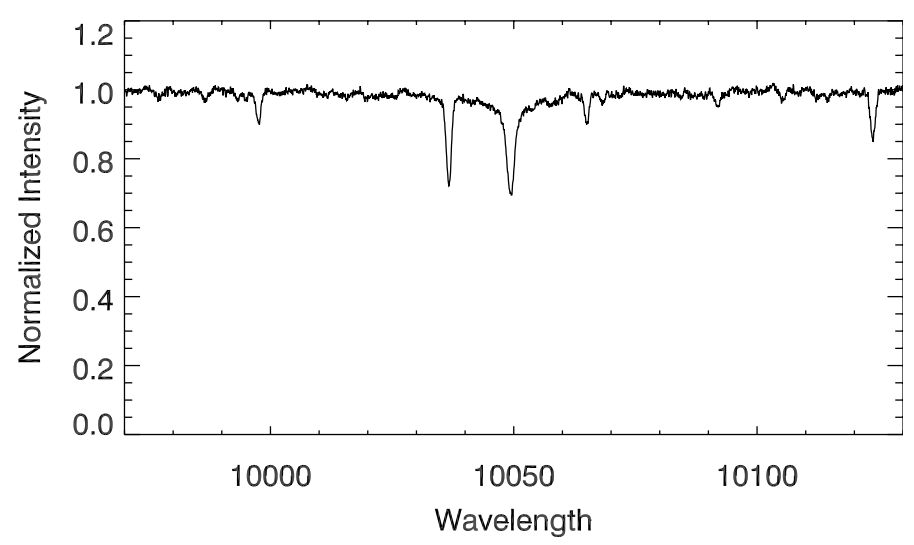

Fig. 49. Profile of $\mathrm{P} \delta$ in HD 187929, F6 Iab.

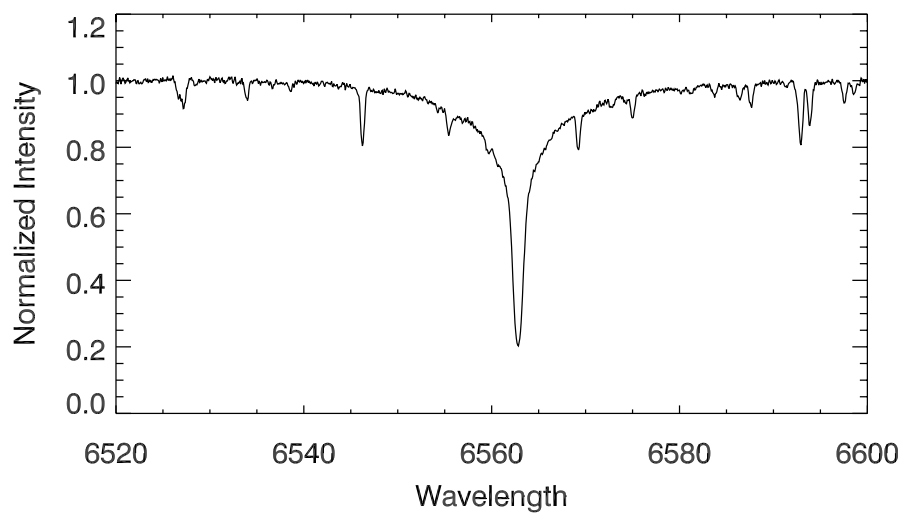

Fig. 50. Profile of $\mathrm{H} \alpha$ in $\mathrm{HD} 33564, \mathrm{~F} 6 \mathrm{~V}$.

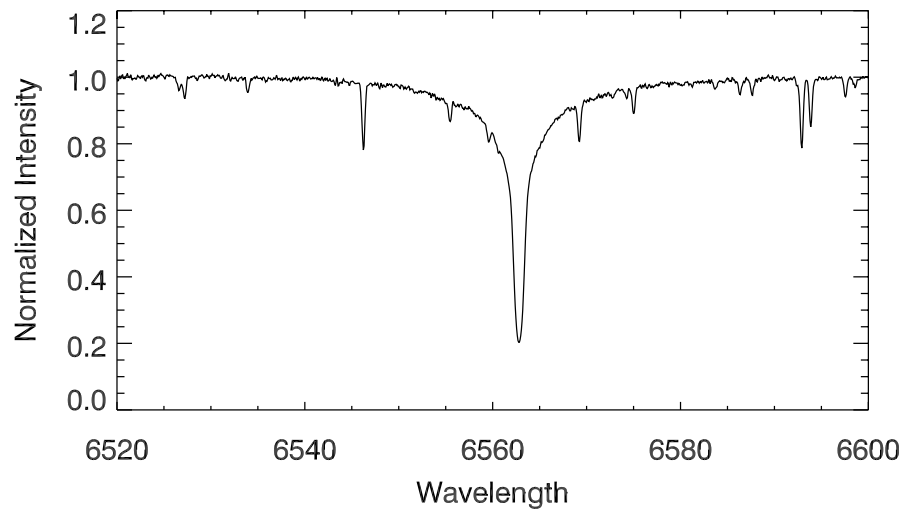

Fig. 51. Profile of $\mathrm{H} \alpha$ in HD 215648, F7 V.
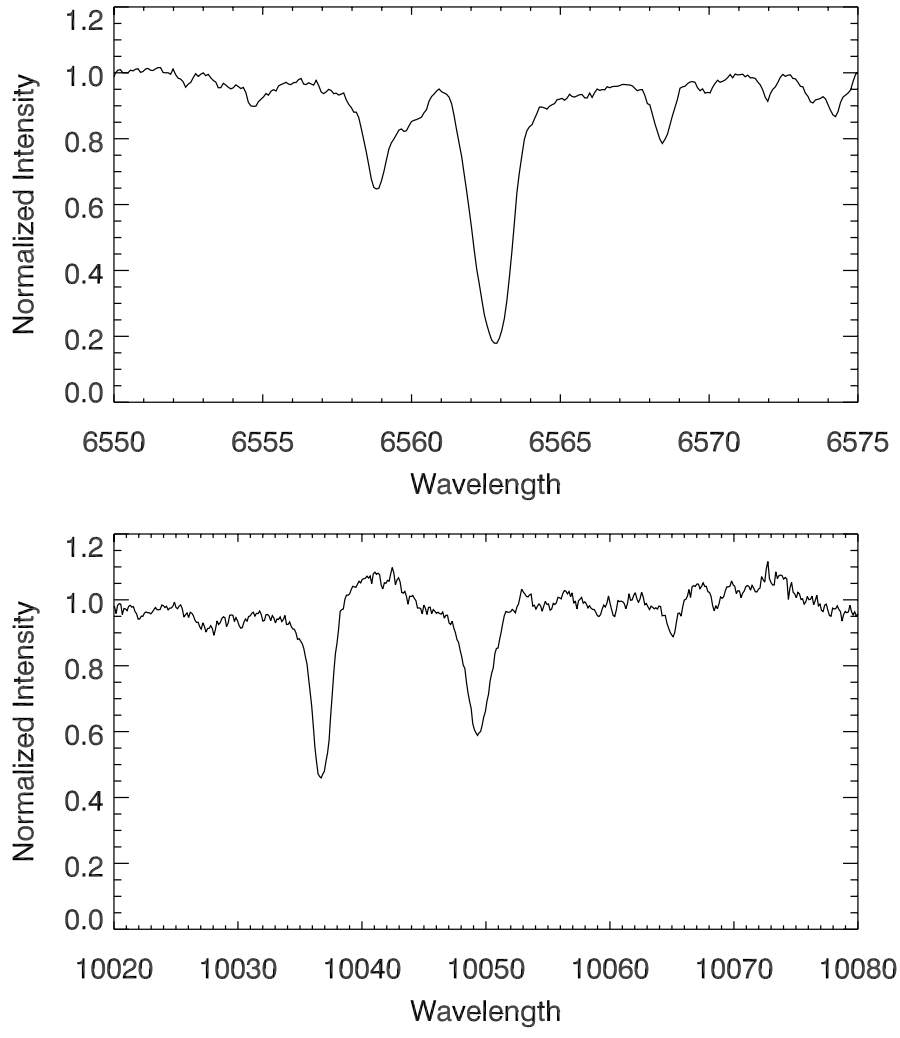

Fig. 52. Profiles of $\mathrm{H} \alpha$ and $\mathrm{P} \delta$ in $\mathrm{HD} 18391, \mathrm{G} 0$ Ia. 
W. Huang et al.: A catalogue of Paschen-line profiles
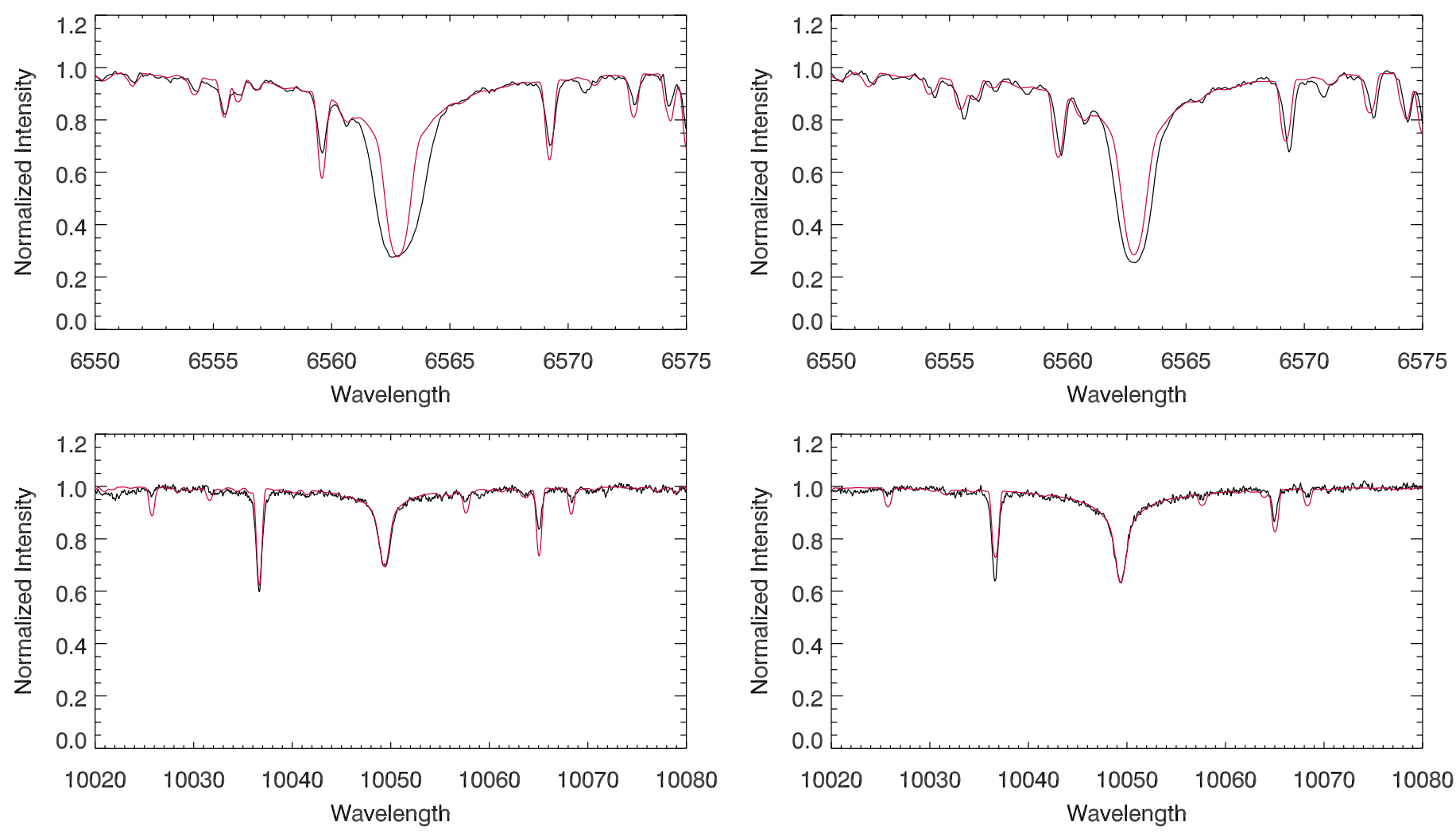

Fig. 53. Profiles of $\mathrm{H} \alpha$ and $\mathrm{P} \delta$ in $\mathrm{HD} 204867, \mathrm{G} 0 \mathrm{Ib}$.

Fig. 55. Profiles of $\mathrm{H} \alpha$ and $\mathrm{P} \delta$ in $\mathrm{HD} 188727$, G5 Ibv.
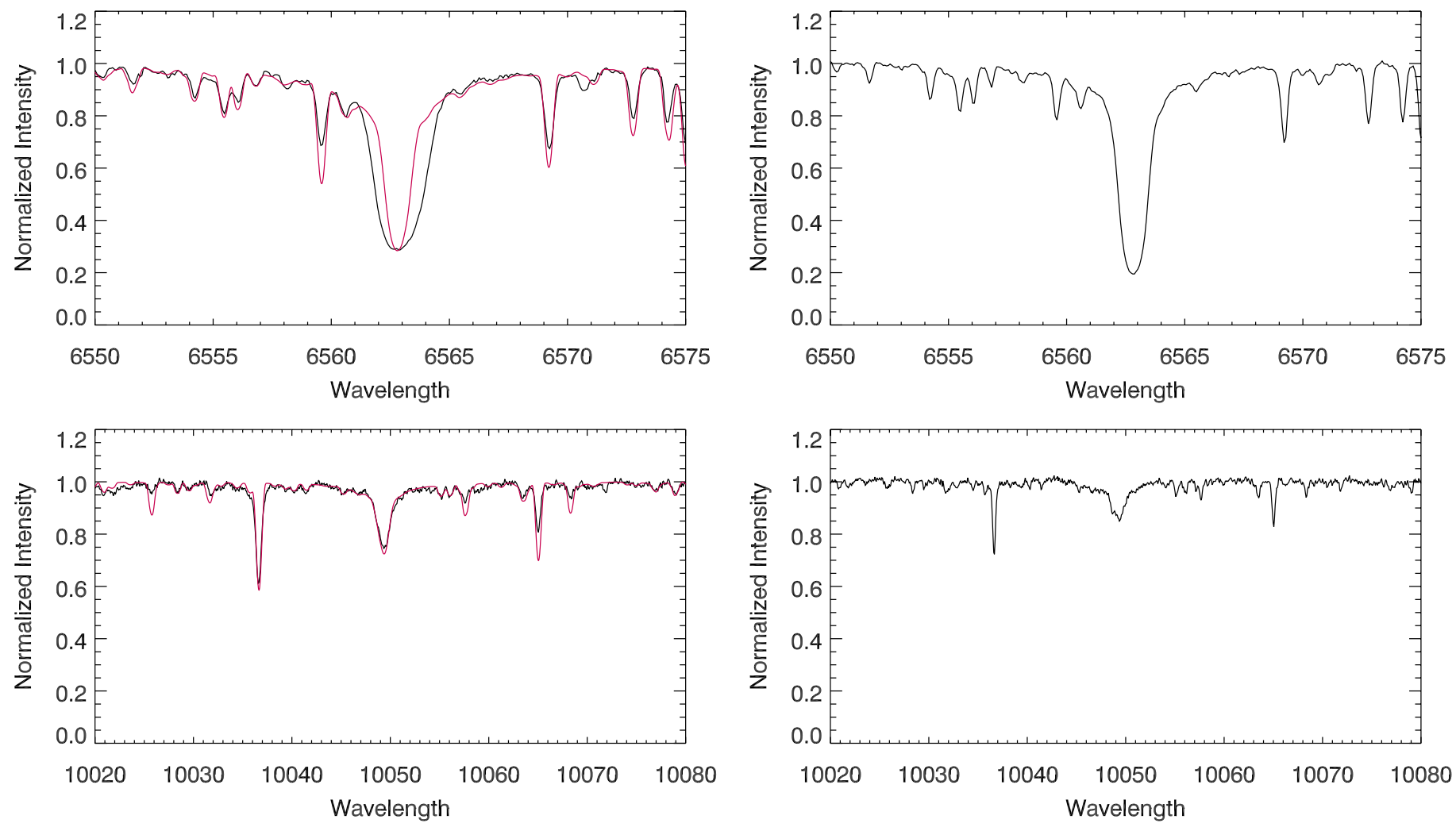

Fig. 54. Profiles of $\mathrm{H} \alpha$ and $\mathrm{P} \delta$ in HD 209750, G2 Ib.

Fig. 56. Profiles of $\mathrm{H} \alpha$ and $\mathrm{P} \delta$ in $\mathrm{HD} 71369$, G5 III. 
A\&A 547, A62 (2012)
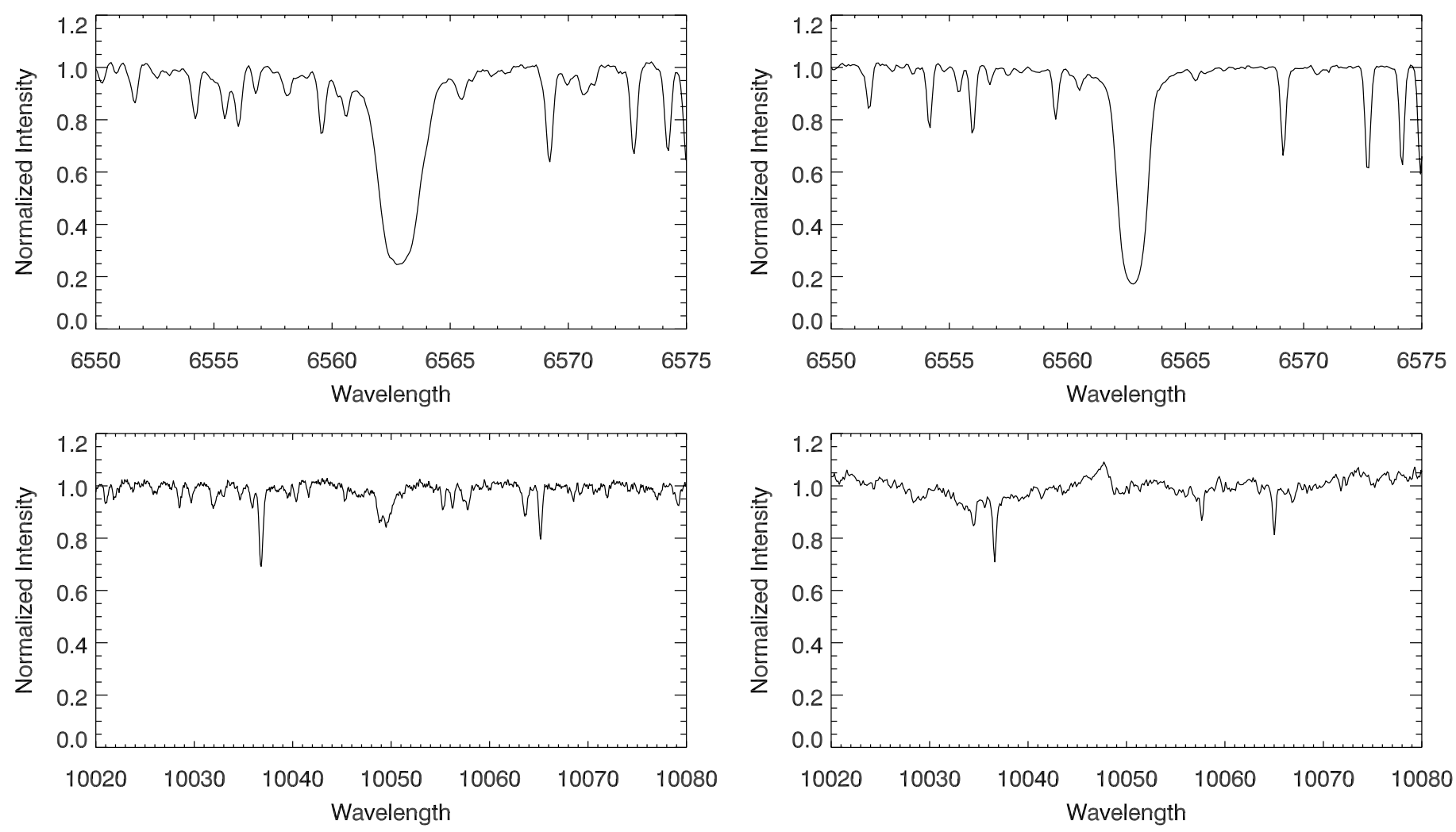

Fig. 57. Profiles of $\mathrm{H} \alpha$ and $\mathrm{P} \delta$ in $\mathrm{HD} 77912$, G8 Ib-II.

Fig. 59. Profiles of $\mathrm{H} \alpha$ and $\mathrm{P} \delta$ in HD 6833, G9 III.
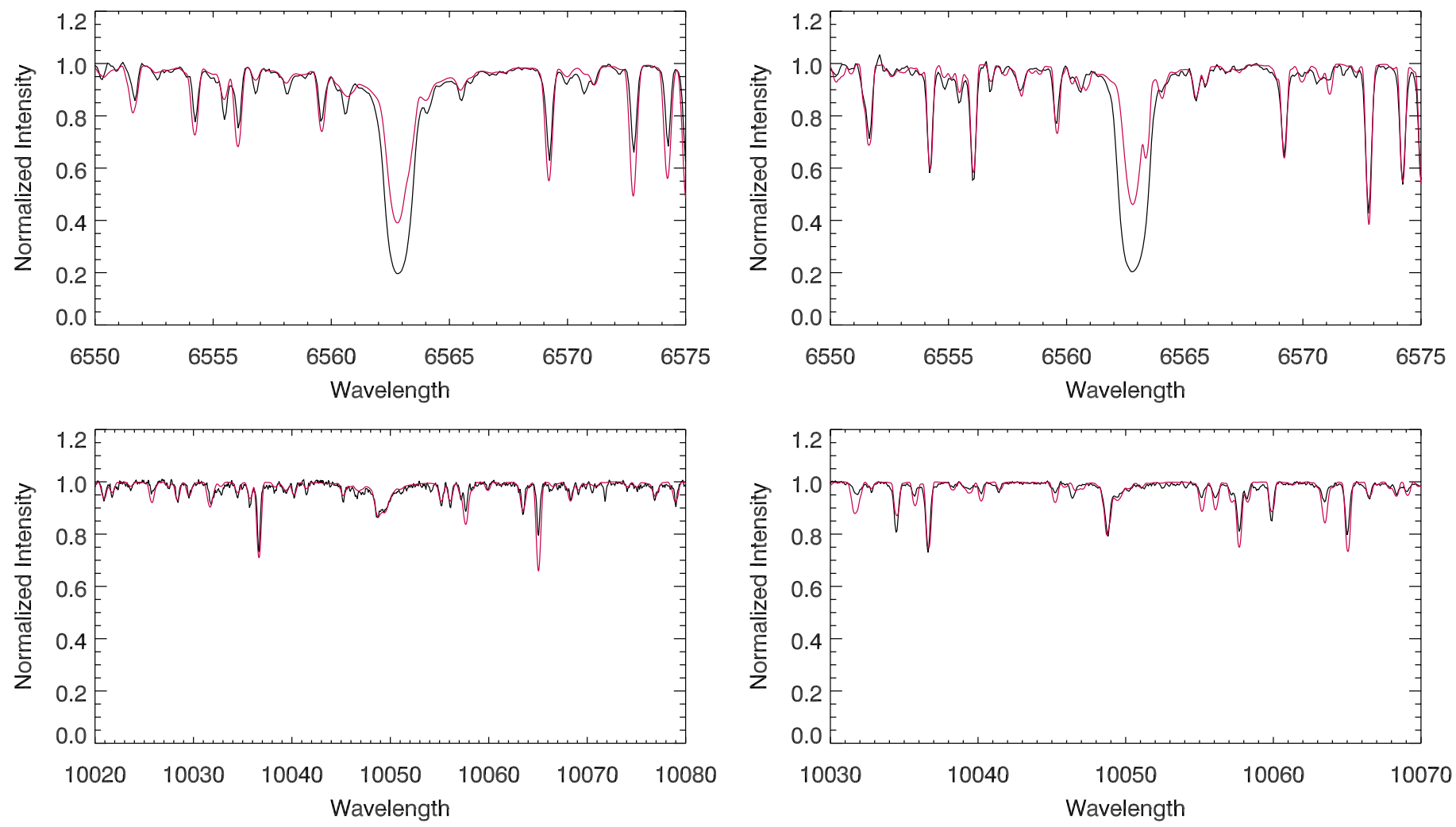

Fig. 58. Profiles of $\mathrm{H} \alpha$ and $\mathrm{P} \delta$ in HD 62345, G8 IIIa.

Fig. 60. Profiles of $\mathrm{H} \alpha$ and $\mathrm{P} \delta$ in HD 124897 (Arcturus) K1.5 III. 
W. Huang et al.: A catalogue of Paschen-line profiles
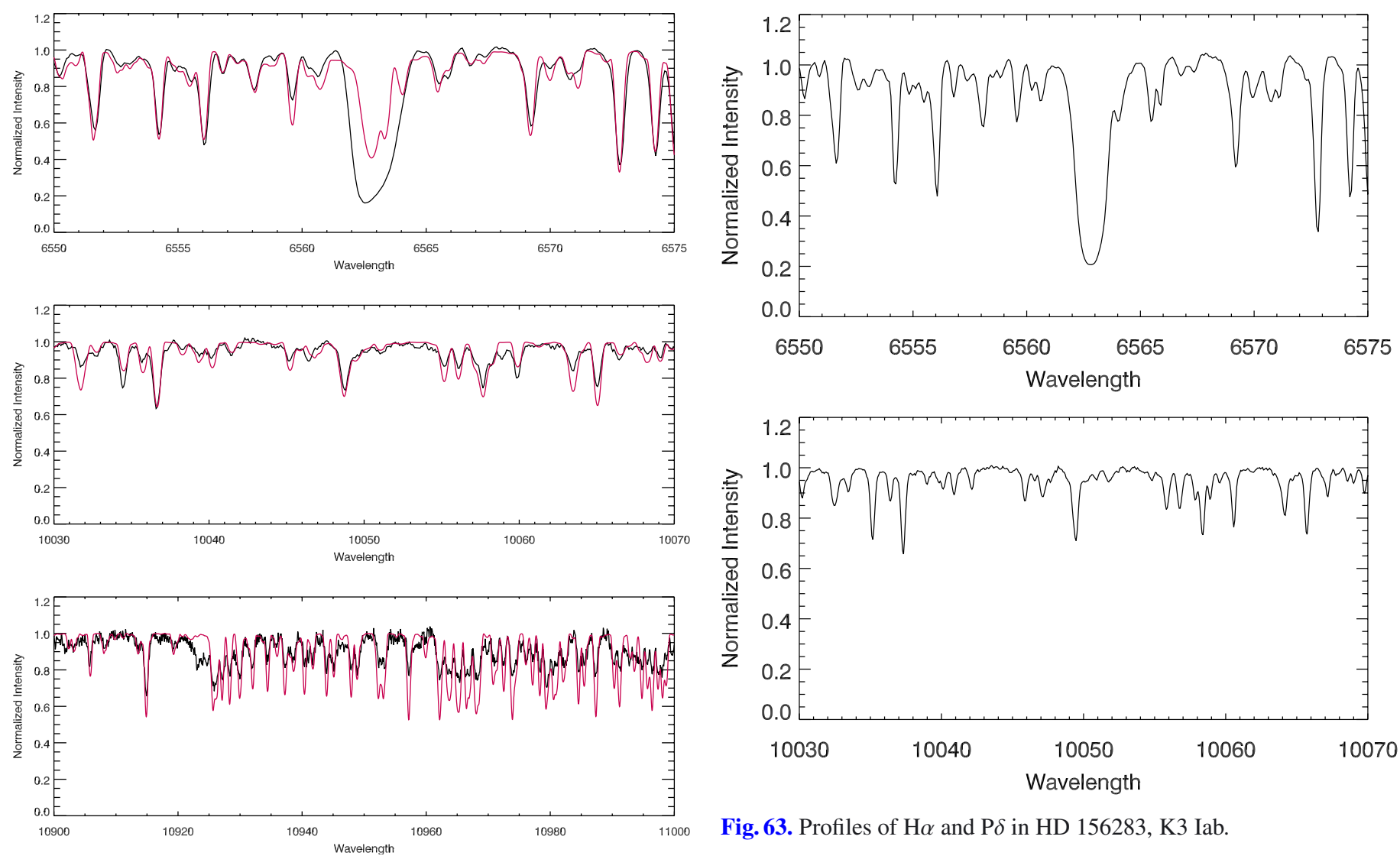

Fig. 63. Profiles of $\mathrm{H} \alpha$ and $\mathrm{P} \delta$ in HD 156283, K3 Iab.

Fig. 61. Profiles of $\mathrm{H} \alpha, \mathrm{P} \delta$, and $\mathrm{P} \gamma$ in $\mathrm{HD} 206778, \mathrm{~K} 2 \mathrm{Ib}$.
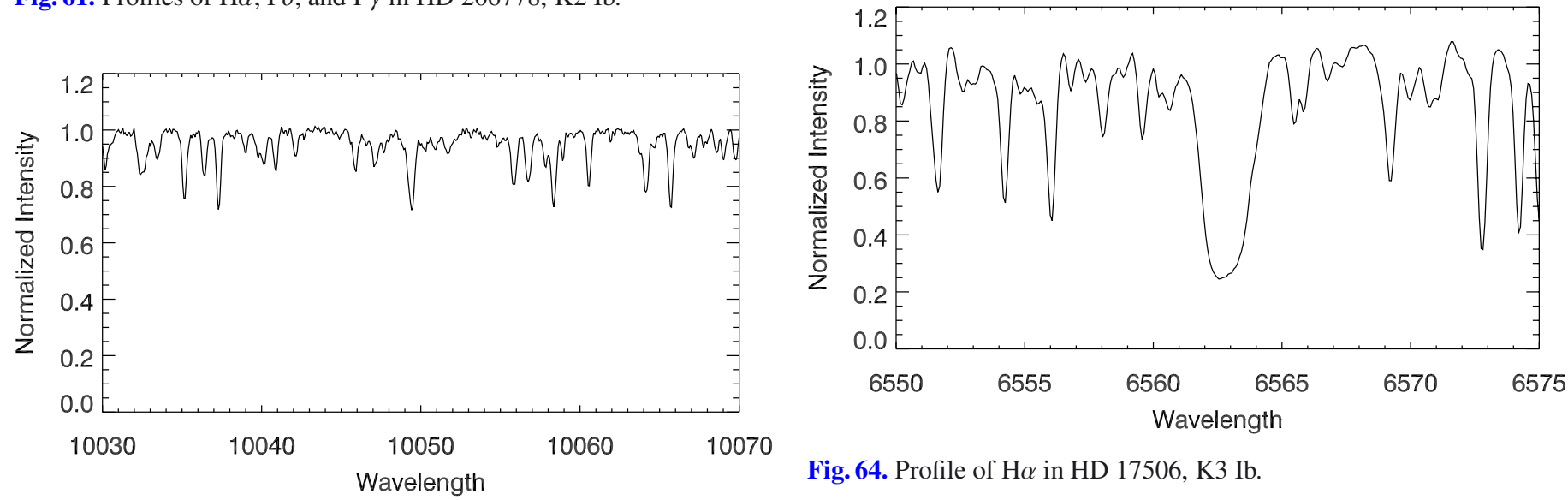

Fig. 64. Profile of $\mathrm{H} \alpha$ in HD 17506, K3 Ib.

Fig. 62. Profile $\mathrm{P} \delta$ in HD 85503, K2 III. 

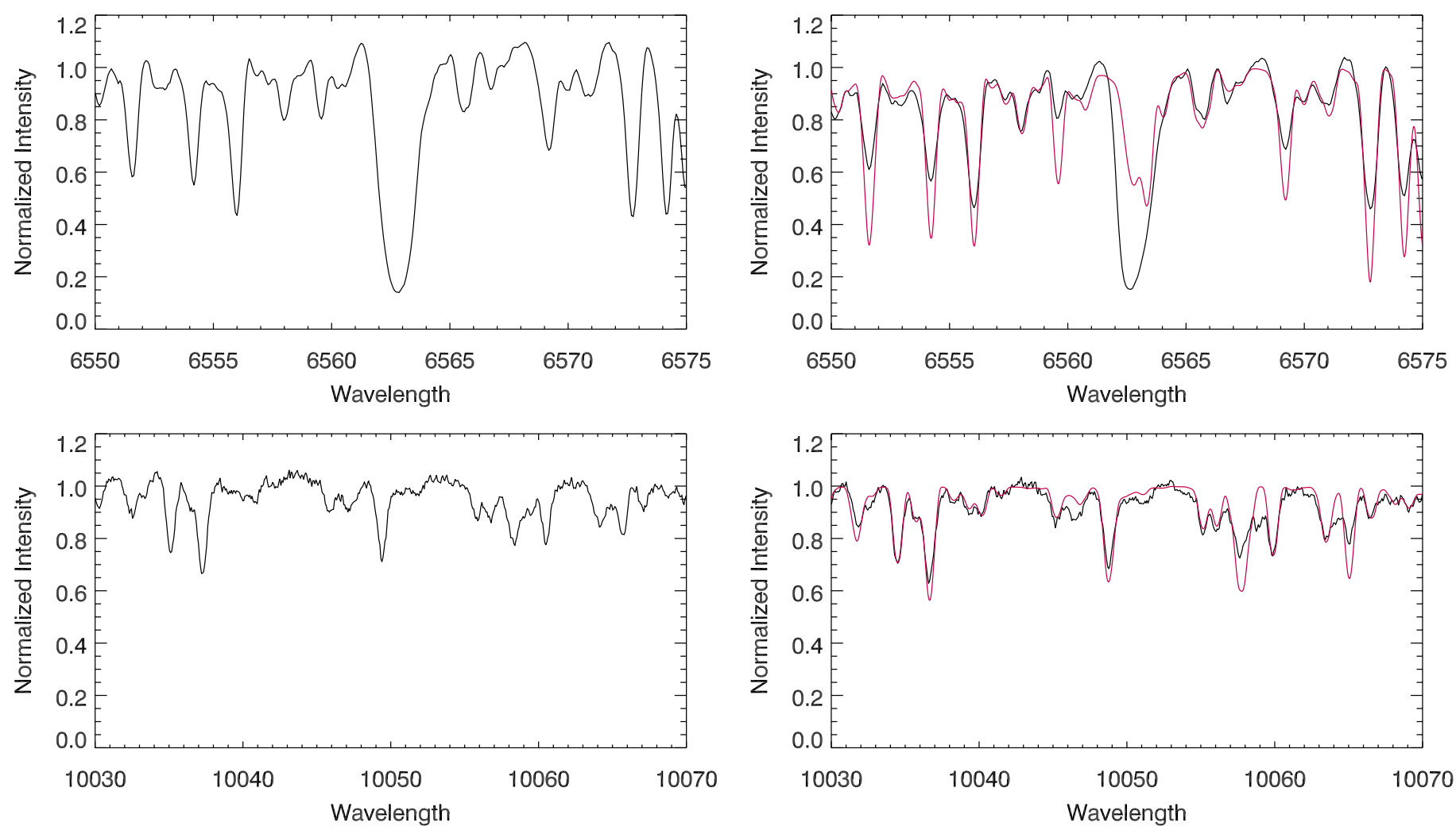

Fig. 65. Profiles of $\mathrm{H} \alpha$ and $\mathrm{P} \delta$ in $\mathrm{HD} 44537, \mathrm{~K} 5 \mathrm{Iab}$.

Fig. 68. Profiles of $\mathrm{H} \alpha$ and $\mathrm{P} \delta$ in HD 42543, M0 Iab.

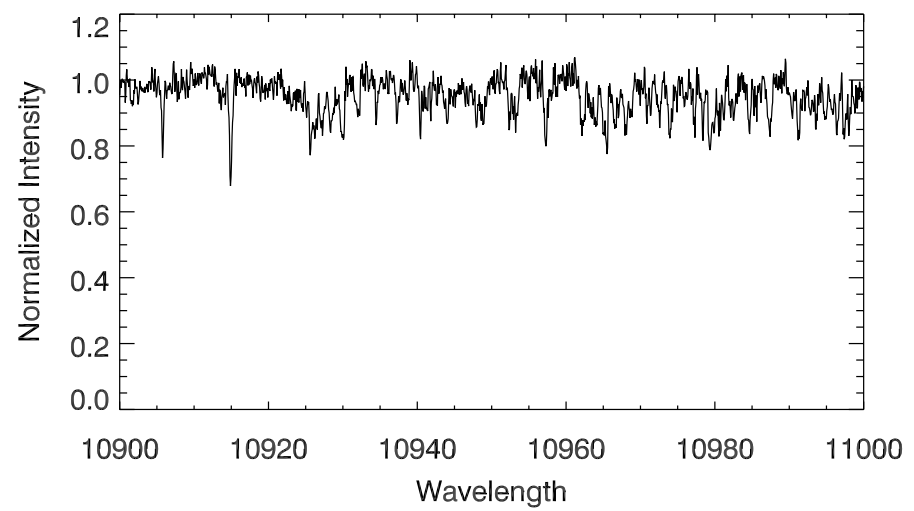

Fig. 66. Profile $\mathrm{P} \gamma$ in HD 29139, K5 III.
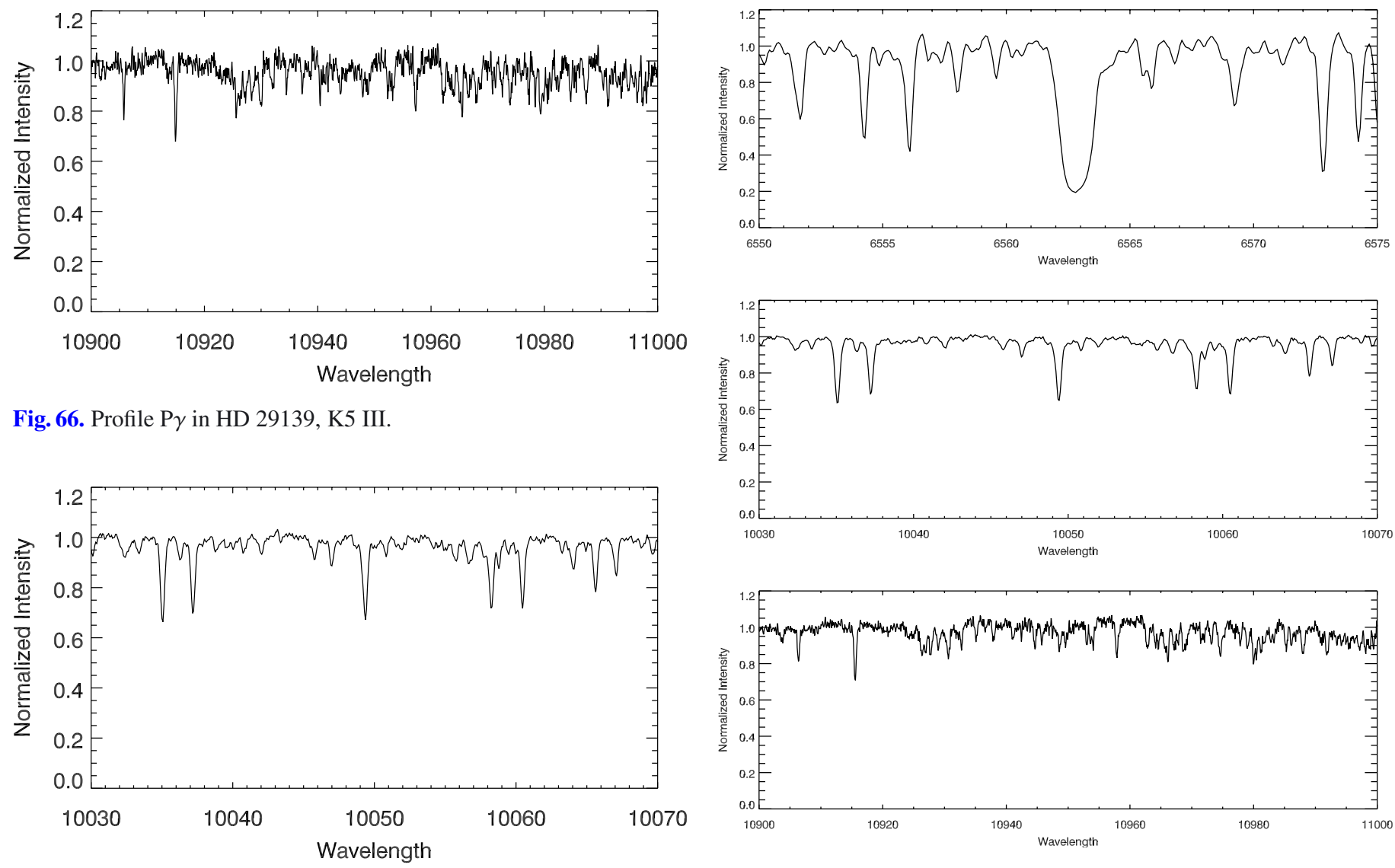

Fig. 67. Profile $\mathrm{P} \delta$ in HD 147767, K5 III.

Fig. 69. Profiles of $\mathrm{H} \alpha, \mathrm{P} \delta$, and $\mathrm{P} \gamma$ in $\mathrm{HD}$ 6860, M0 III. 
W. Huang et al.: A catalogue of Paschen-line profiles
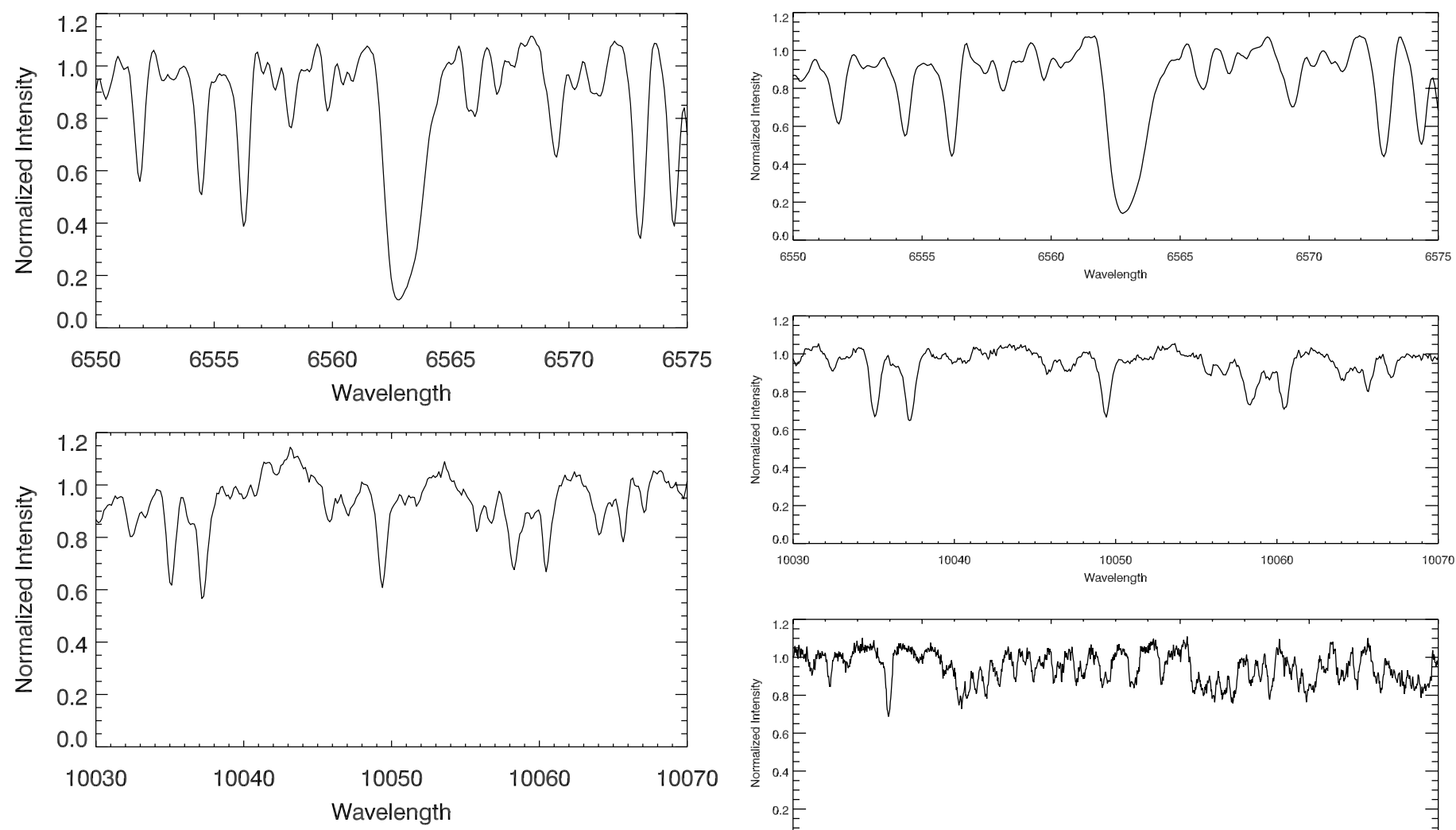

Fig. 70. Profiles of $\mathrm{H} \alpha$ and $\mathrm{P} \delta$ in $\mathrm{HD} 14330, \mathrm{M} 1 \mathrm{sd}$.
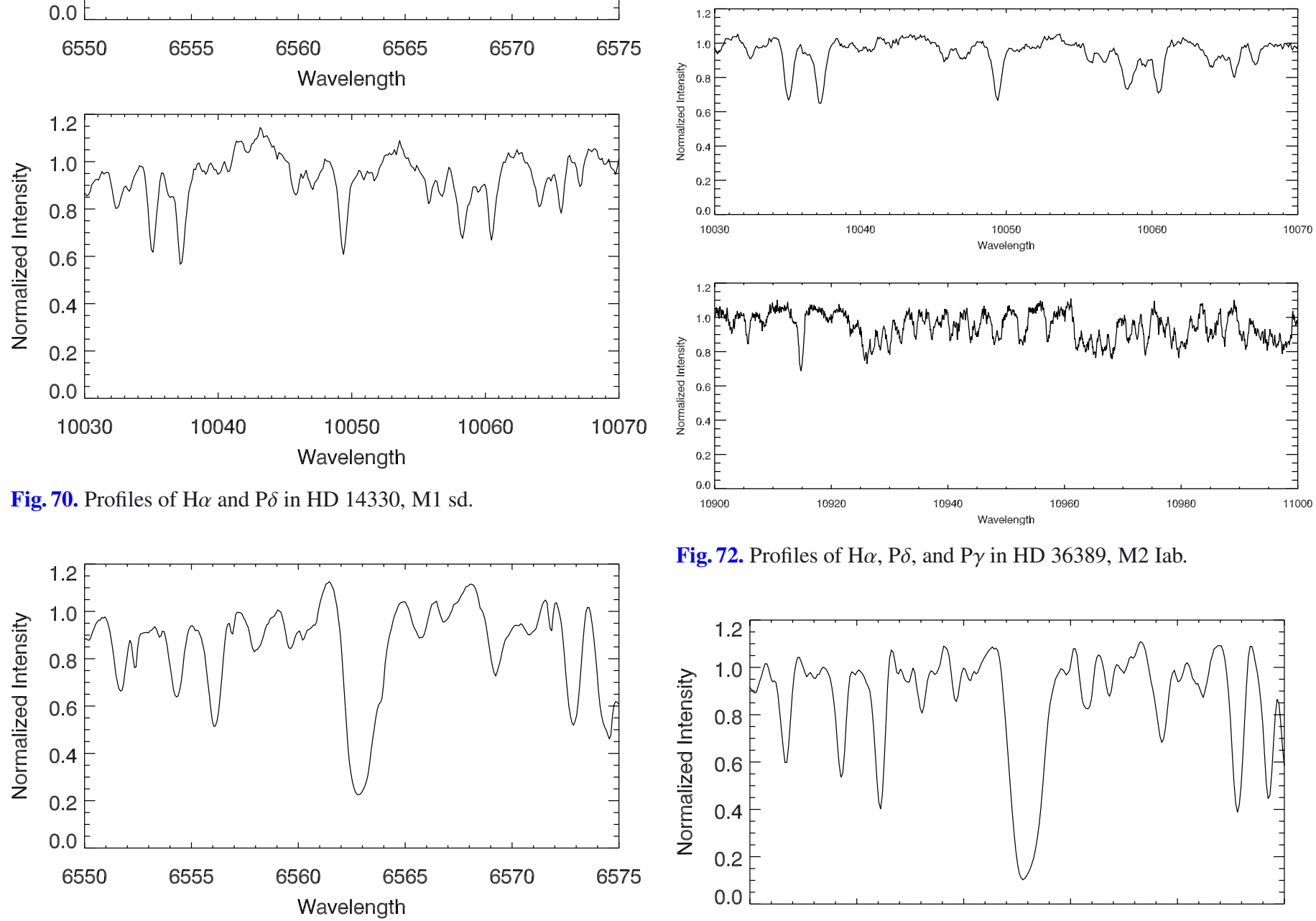

Fig. 72. Profiles of $\mathrm{H} \alpha, \mathrm{P} \delta$, and $\mathrm{P} \gamma$ in $\mathrm{HD} 36389, \mathrm{M} 2 \mathrm{Iab}$.
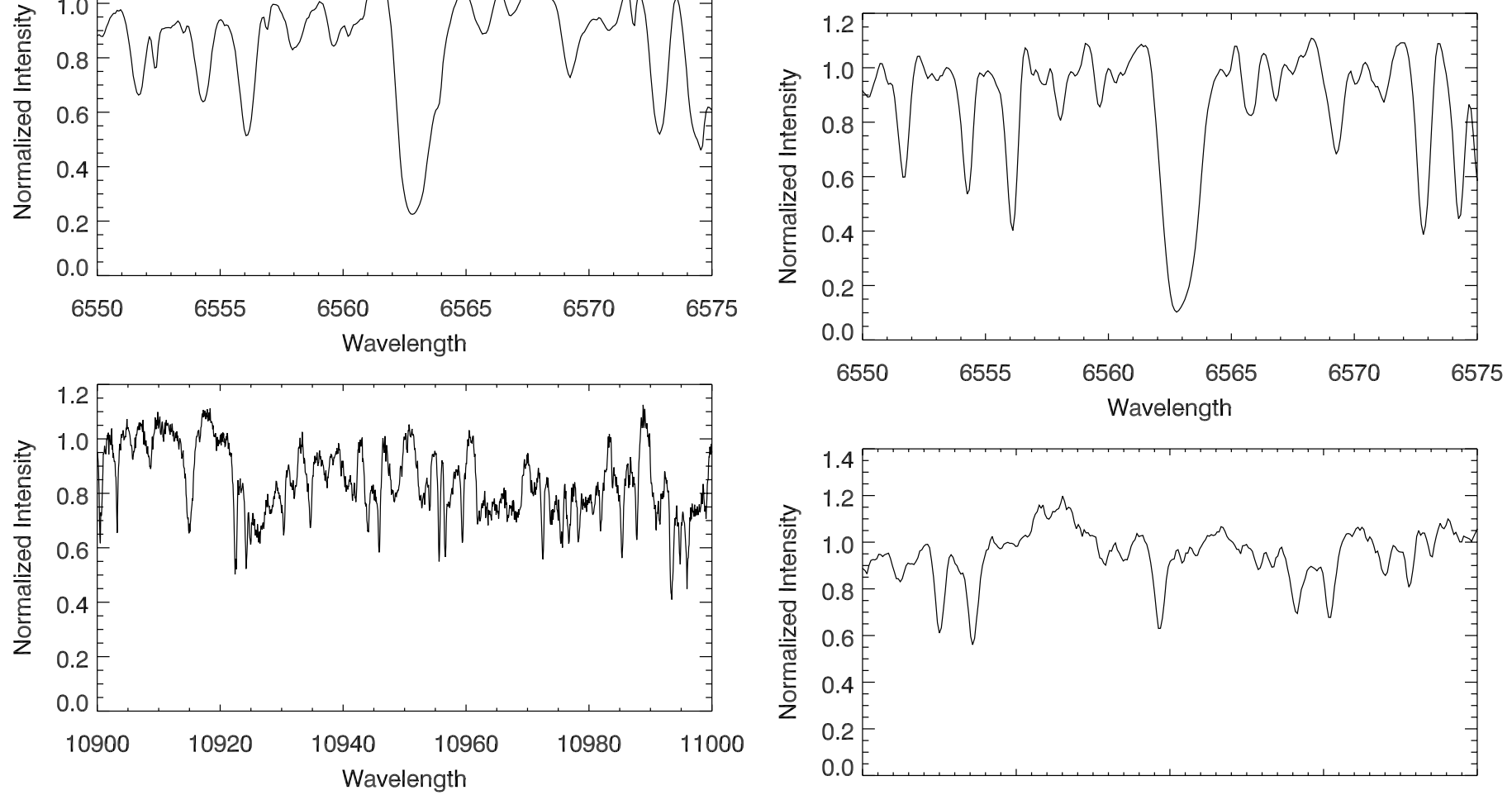

Fig. 71. Profiles of $\mathrm{H} \alpha$ and $\mathrm{P} \gamma$ in HD 206936 ( $\mu$ Cep) M2 Ia.

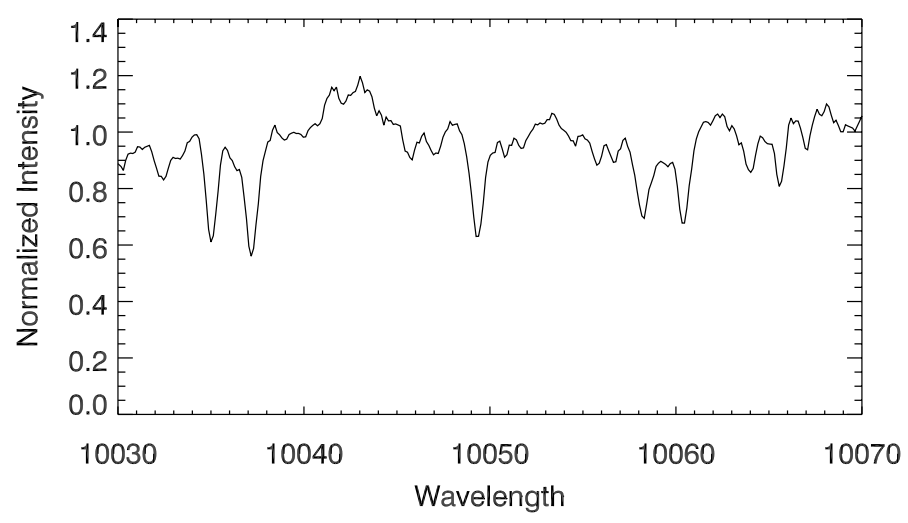

Fig. 73. Profiles of $\mathrm{H} \alpha$ and $\mathrm{P} \delta$ in $\mathrm{HD} 13136, \mathrm{M} 2 \mathrm{Iab}$. 


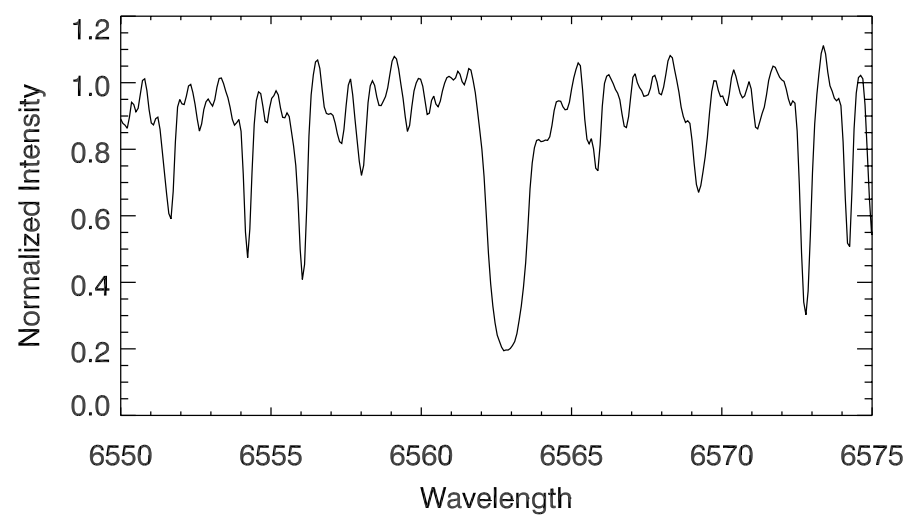

Fig. 74. Profile of $\mathrm{H} \alpha$ in HD 147749, M2 III.
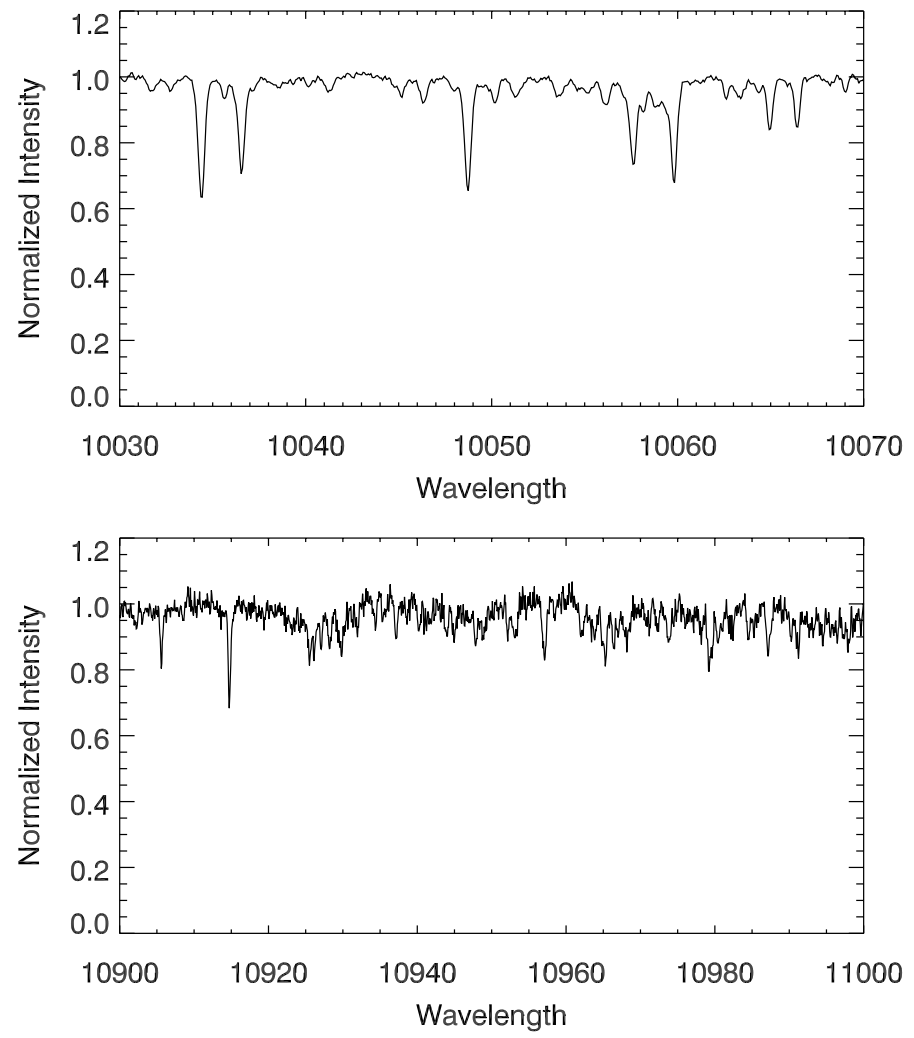

Fig. 75. Profiles of $\mathrm{P} \delta$ and $\mathrm{P} \gamma$ in HD 217906, M2.5 II.

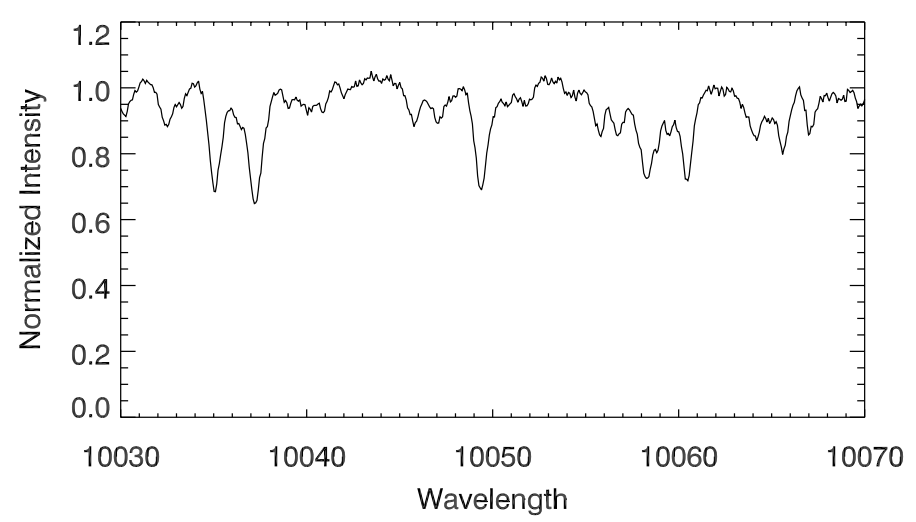

Fig. 76. Profile $\mathrm{P} \delta$ in HD 14469, M 3 Iab.

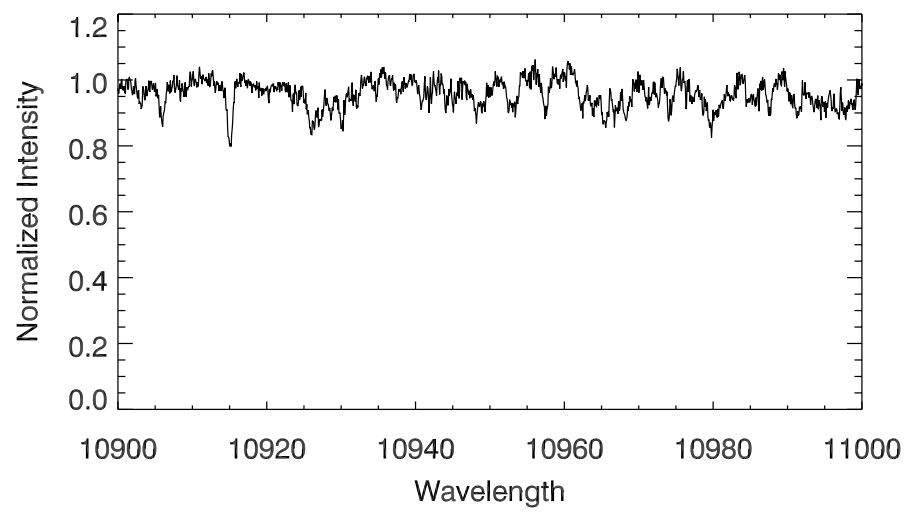

Fig. 77. Profile of P $\gamma$ in HD 42995, M3 III.

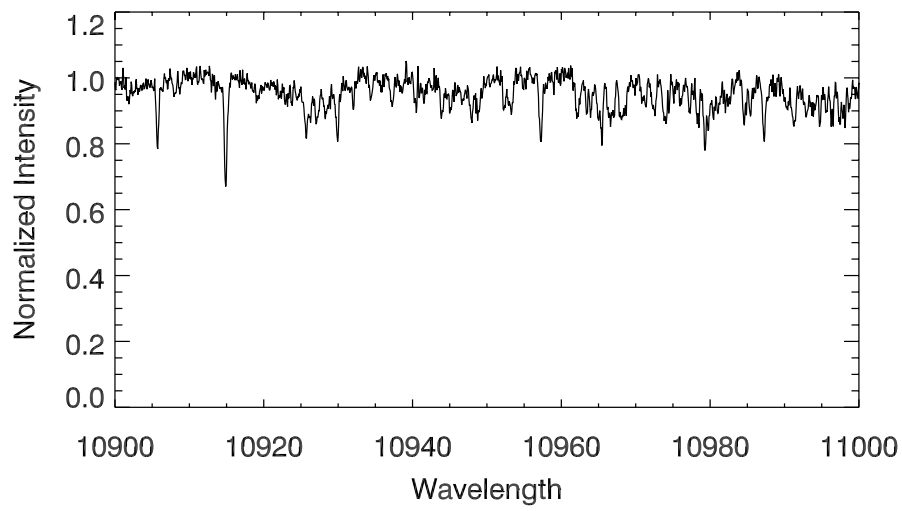

Fig. 78. Profile of $\mathrm{P} \gamma$ in HD 44478, M3 III.
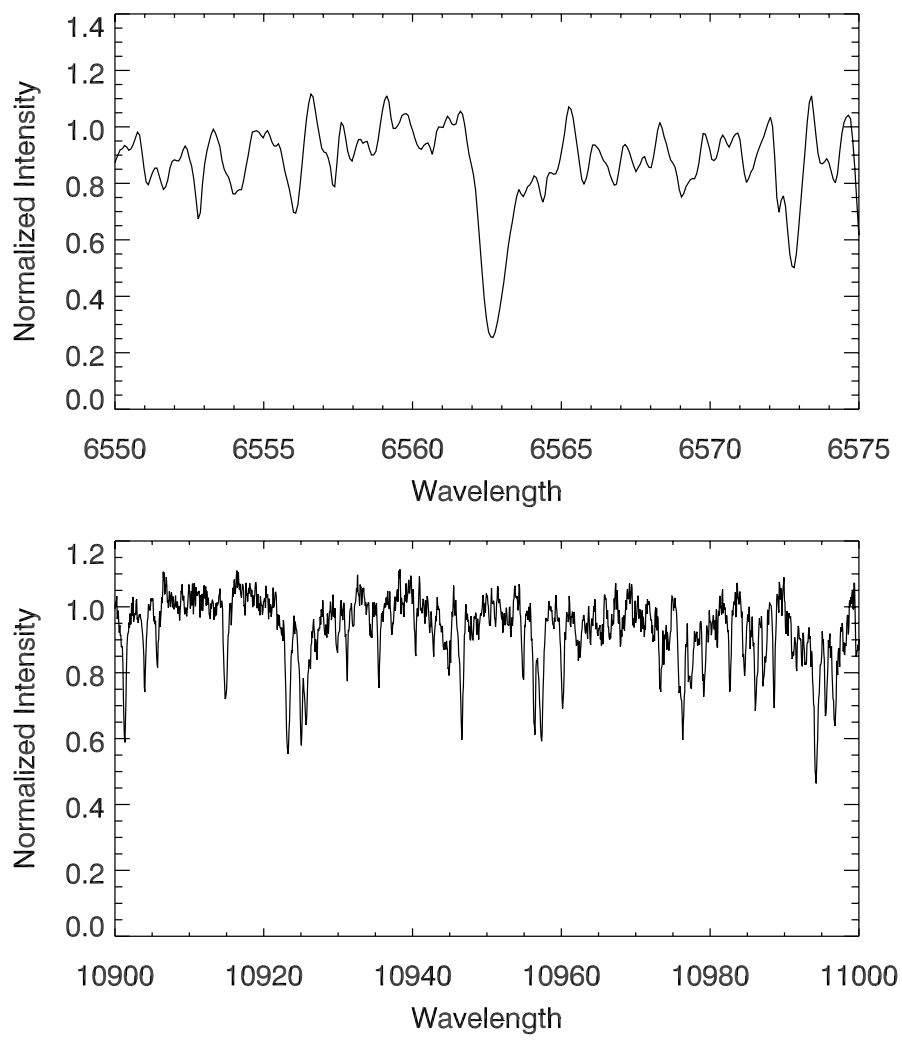

Fig. 79. Profiles of $\mathrm{H} \alpha$ and $\mathrm{P} \gamma$ in HD 172380 (XY Lyr) M4 Iab. 
W. Huang et al.: A catalogue of Paschen-line profiles
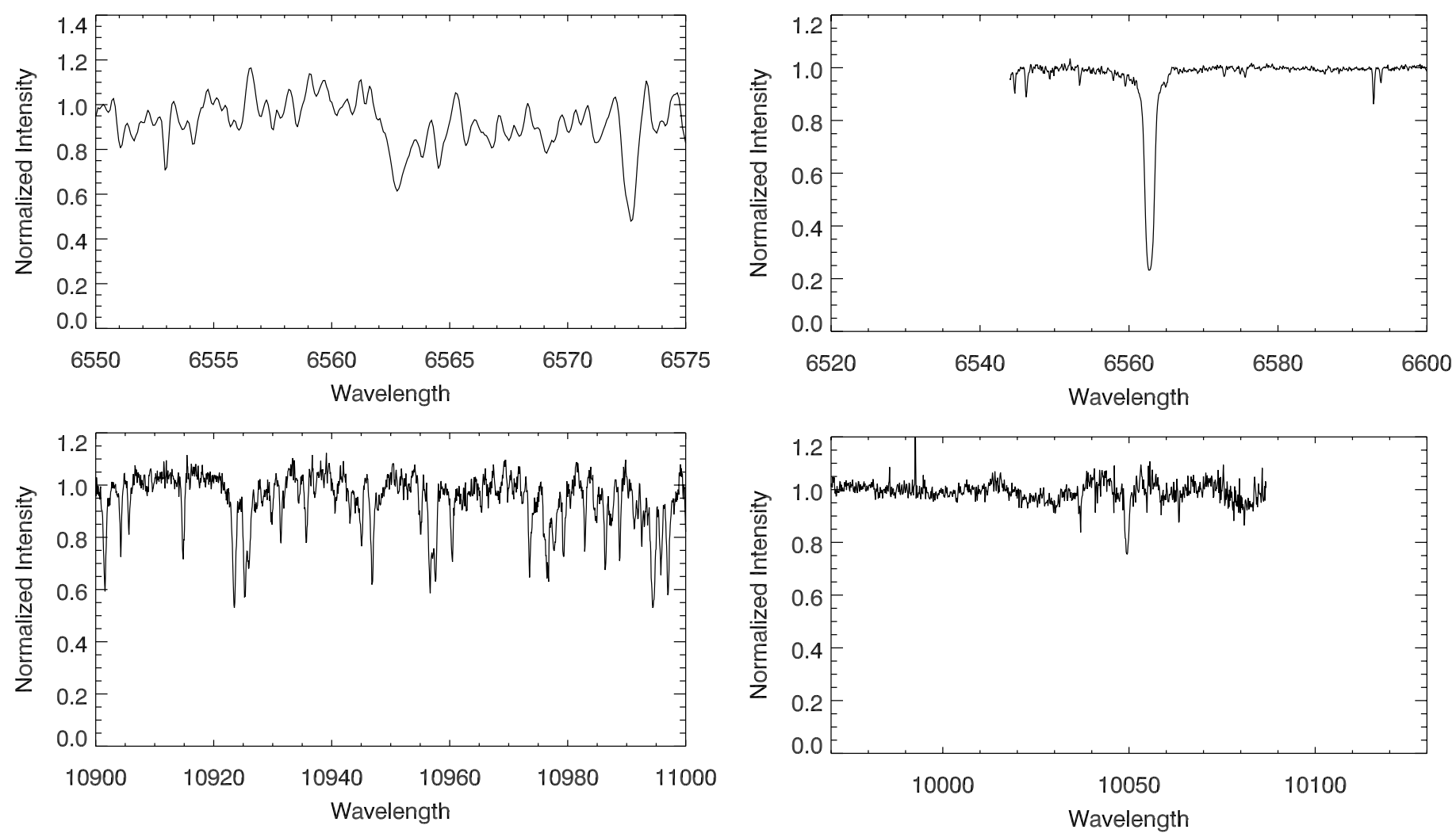

Fig. 80. Profiles of $\mathrm{H} \alpha$ and $\mathrm{P} \gamma$ in HD 197812 (U Del) M5 Ia.

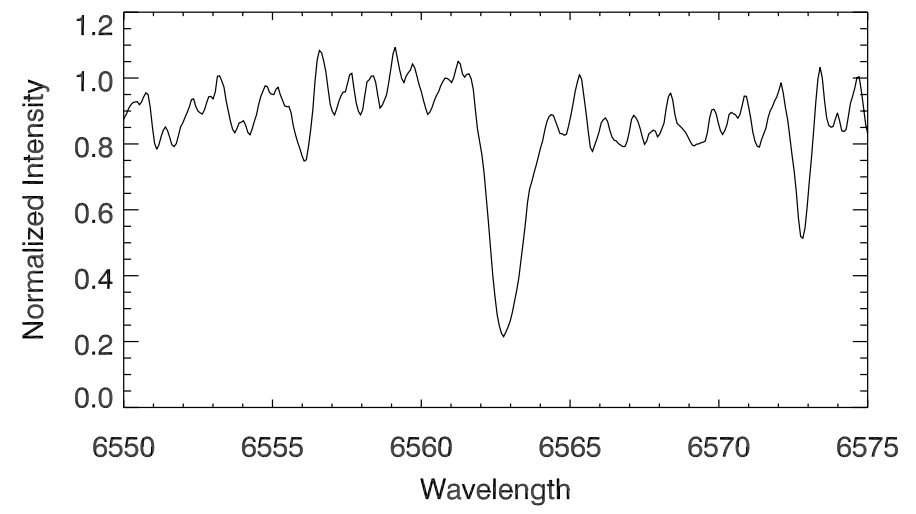

Fig. 82. Profiles of $\mathrm{H} \alpha$ and $\mathrm{P} \delta$ in HD 2796, F p. The limited wavelength coverage is due to the display of one order of the echelle.
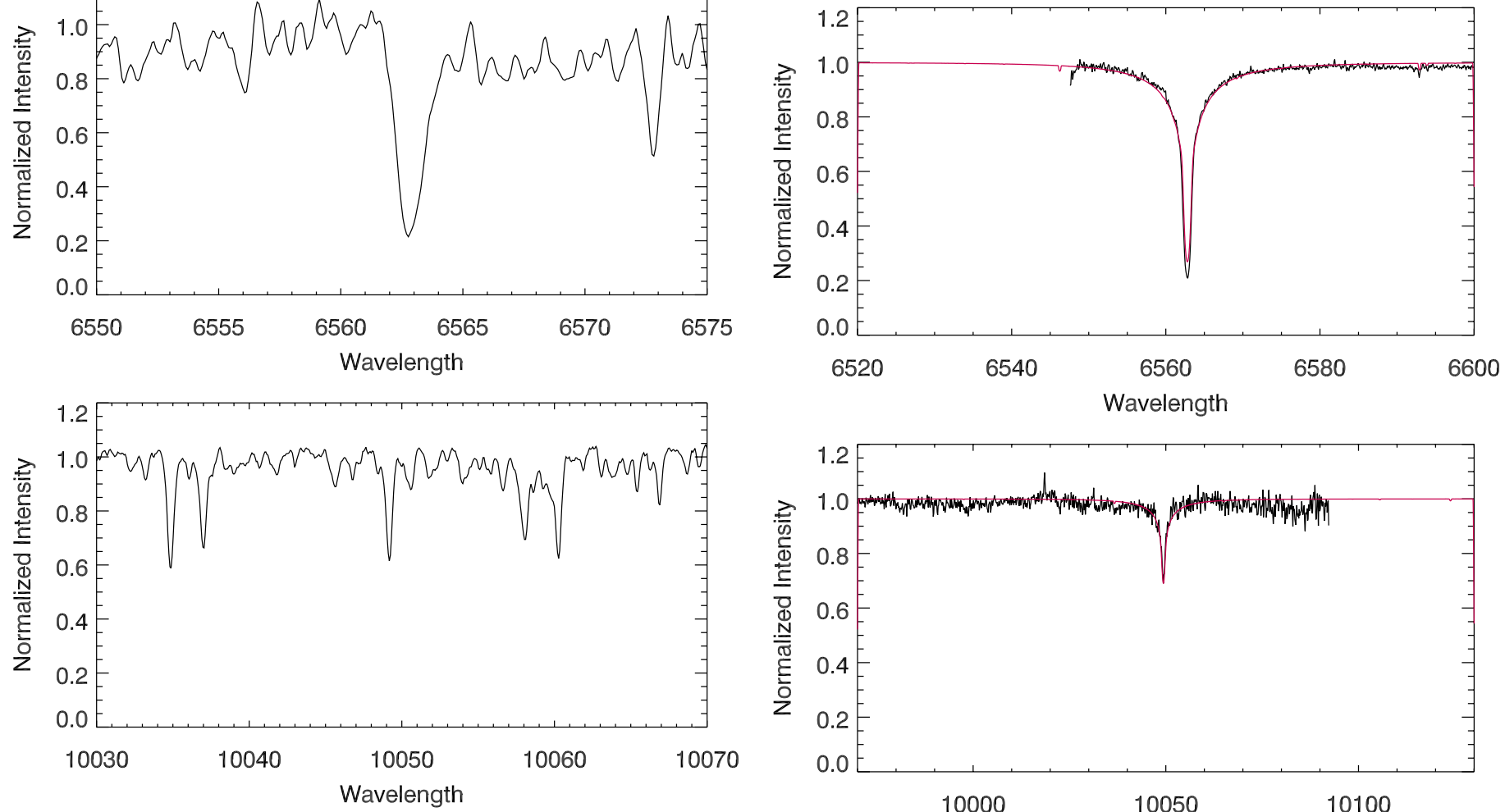

Fig. 81. Profiles of $\mathrm{H} \alpha$ and $\mathrm{P} \delta$ in HD 156014, M5 Ib-II.

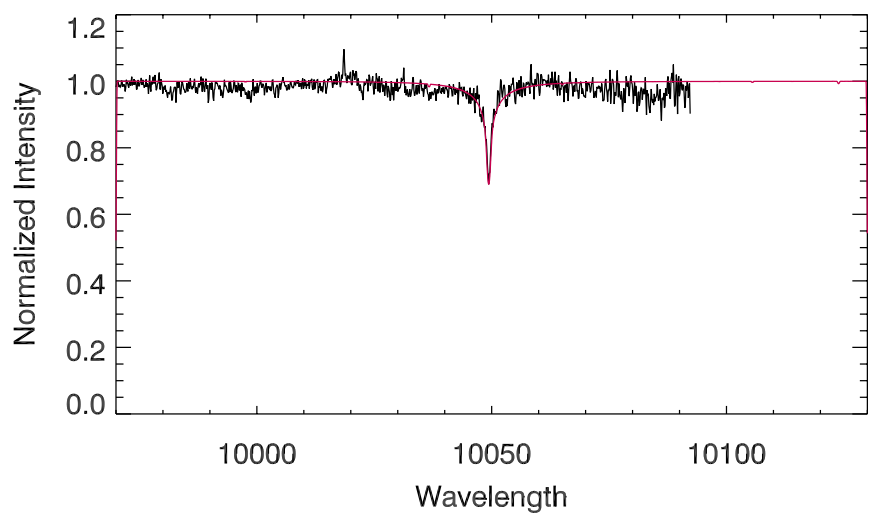

Fig. 83. Profiles of $\mathrm{H} \alpha$ and $\mathrm{P} \delta$ in HD 140283, F3 sd. The limited wavelength coverage is due to the display of one order of the echelle. 
A\&A 547, A62 (2012)
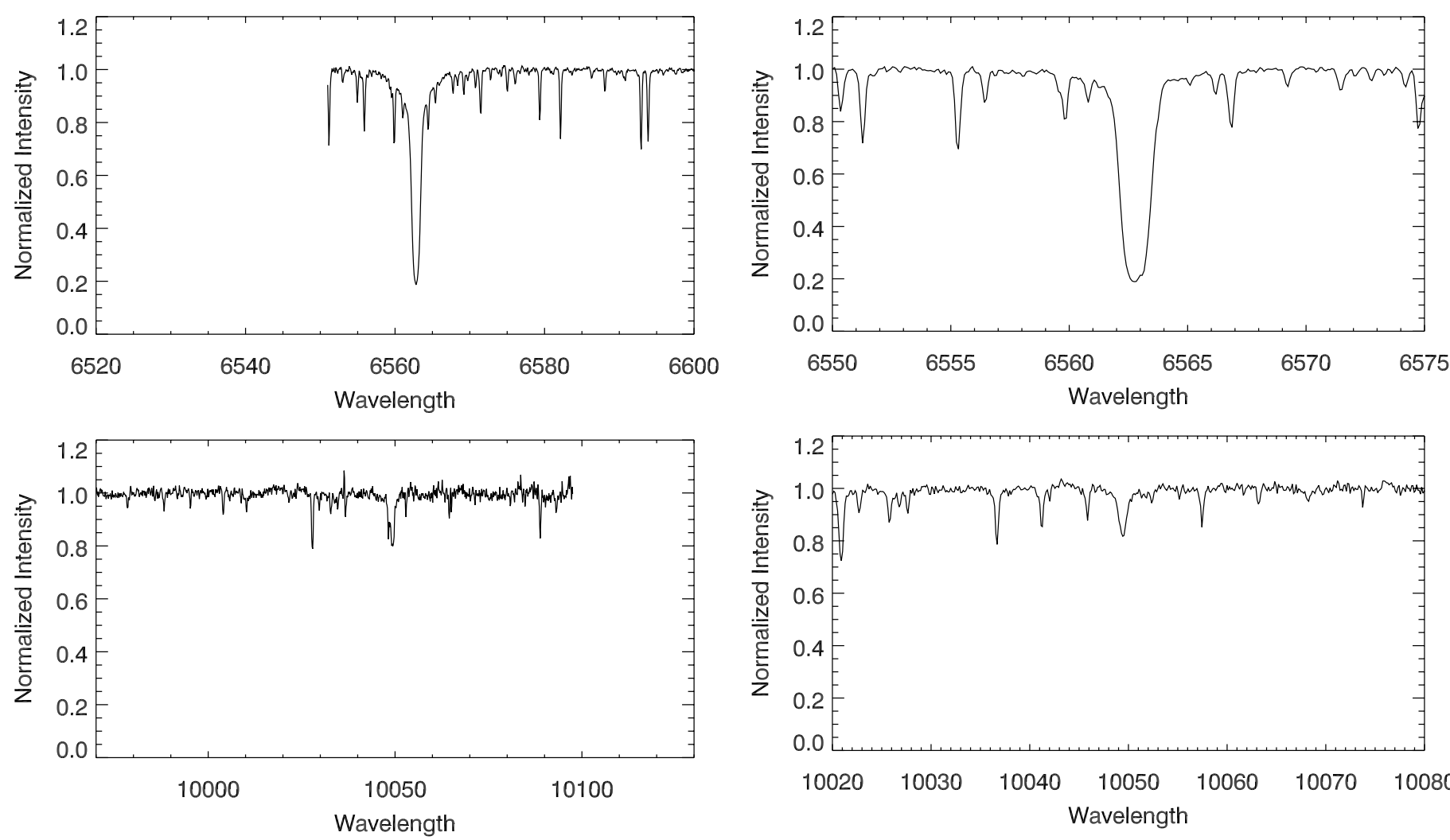

Fig. 84. Profiles of $\mathrm{H} \alpha$ and $\mathrm{P} \delta$ in HD 6755, F8 V. The limited wavelength coverage is due to the display of one order of the echelle.
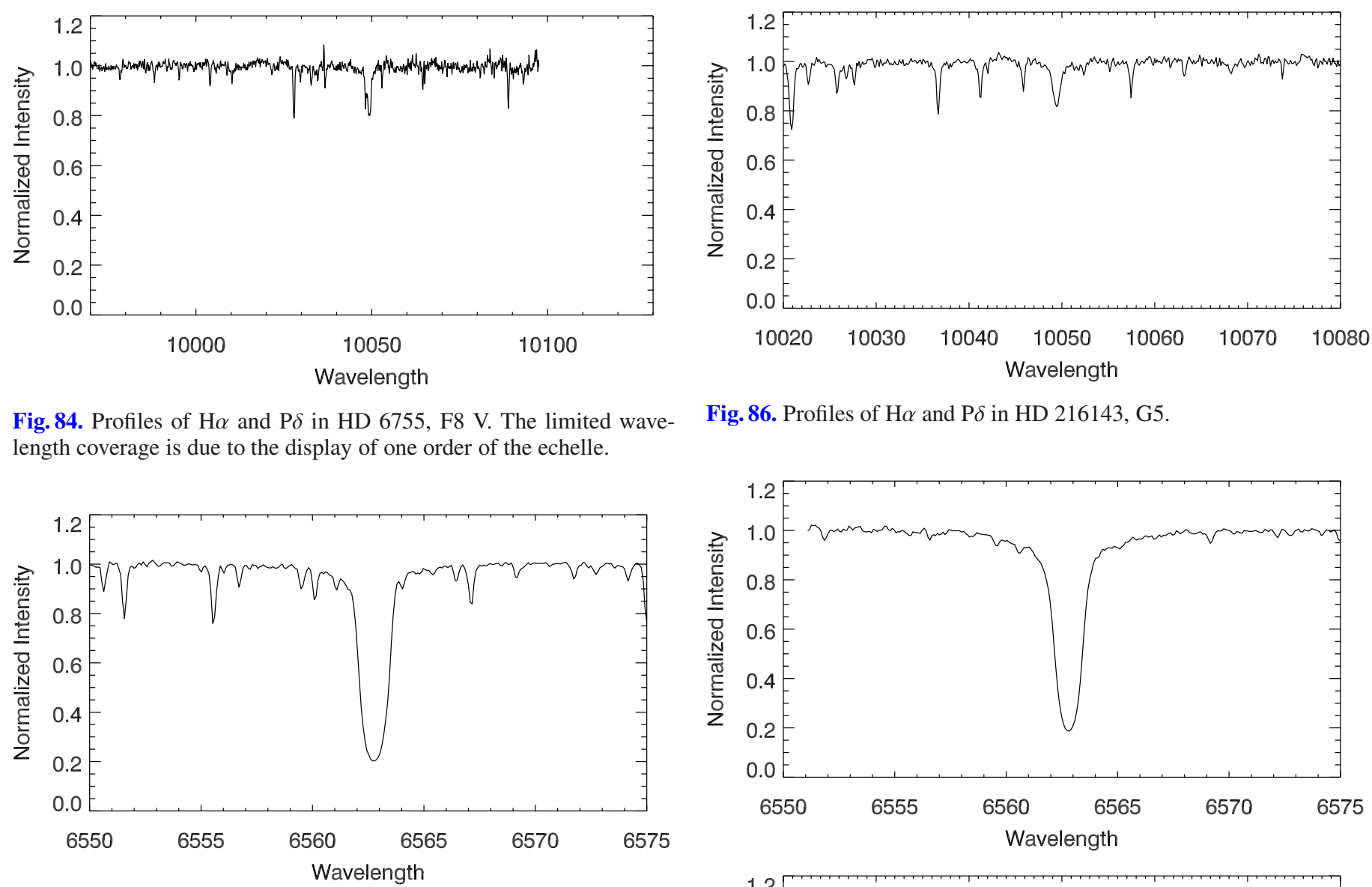

Fig. 86. Profiles of $\mathrm{H} \alpha$ and $\mathrm{P} \delta$ in HD 216143, G5.
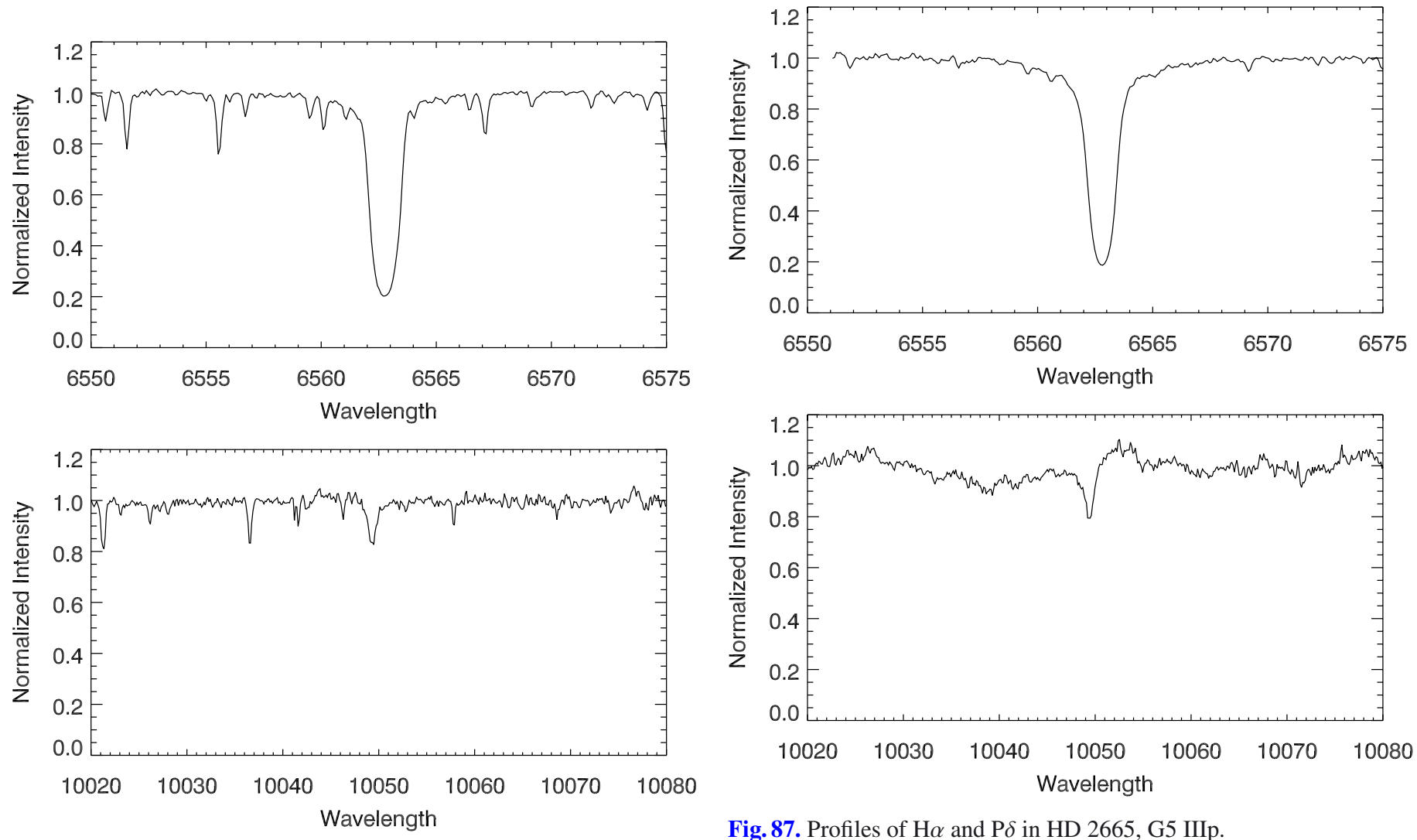

Fig. 87. Profiles of $\mathrm{H} \alpha$ and $\mathrm{P} \delta$ in HD 2665, G5 IIIp.

Fig. 85. Profiles of $\mathrm{H} \alpha$ and $\mathrm{P} \delta$ in HD 221170, G2 IV. 
W. Huang et al.: A catalogue of Paschen-line profiles
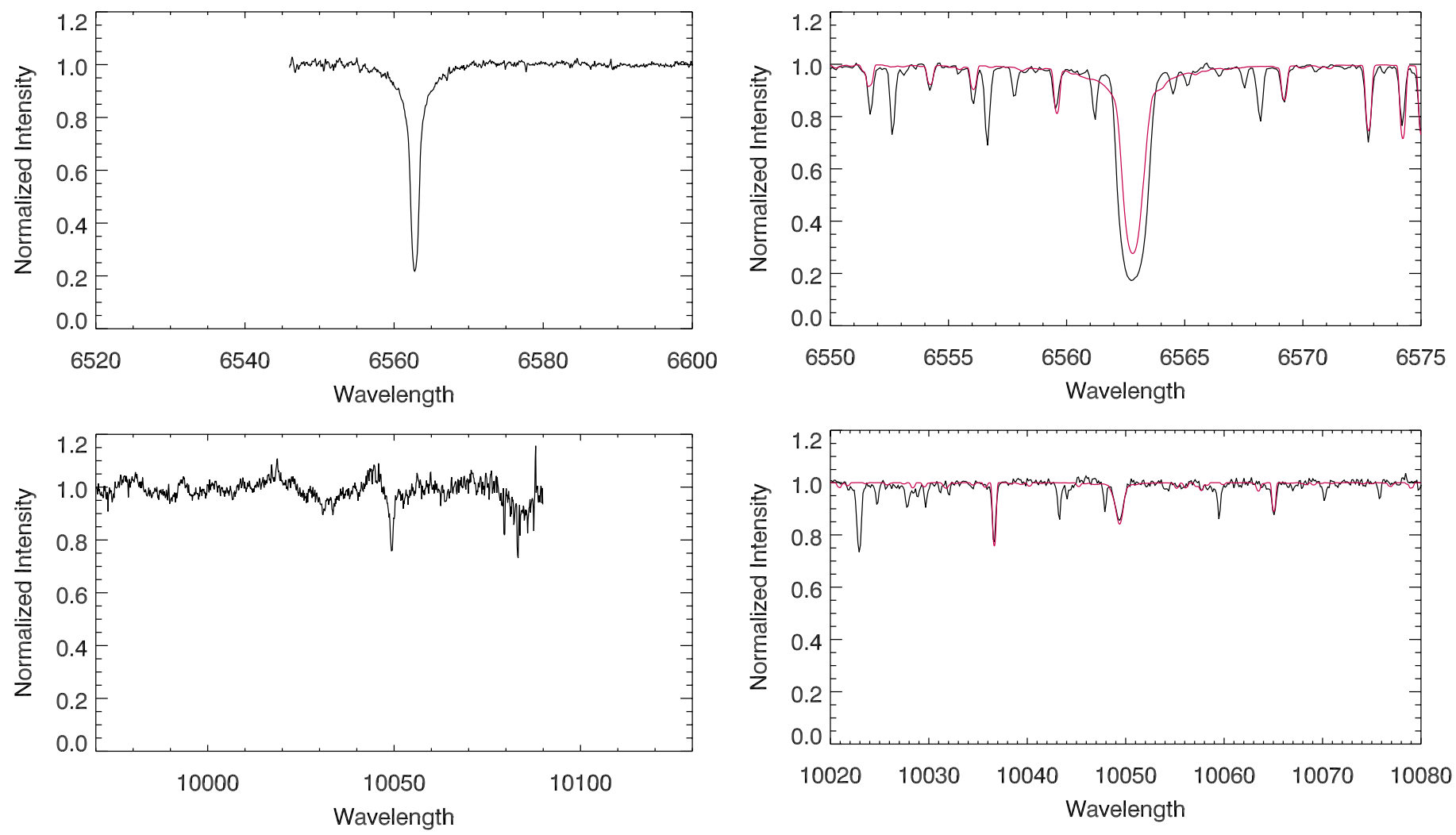

Fig. 88. Profiles of $\mathrm{H} \alpha$ and $\mathrm{P} \delta$ in $\mathrm{BD}+44493$, G5 IV. The limited wavelength coverage is due to the display of one order of the echelle.
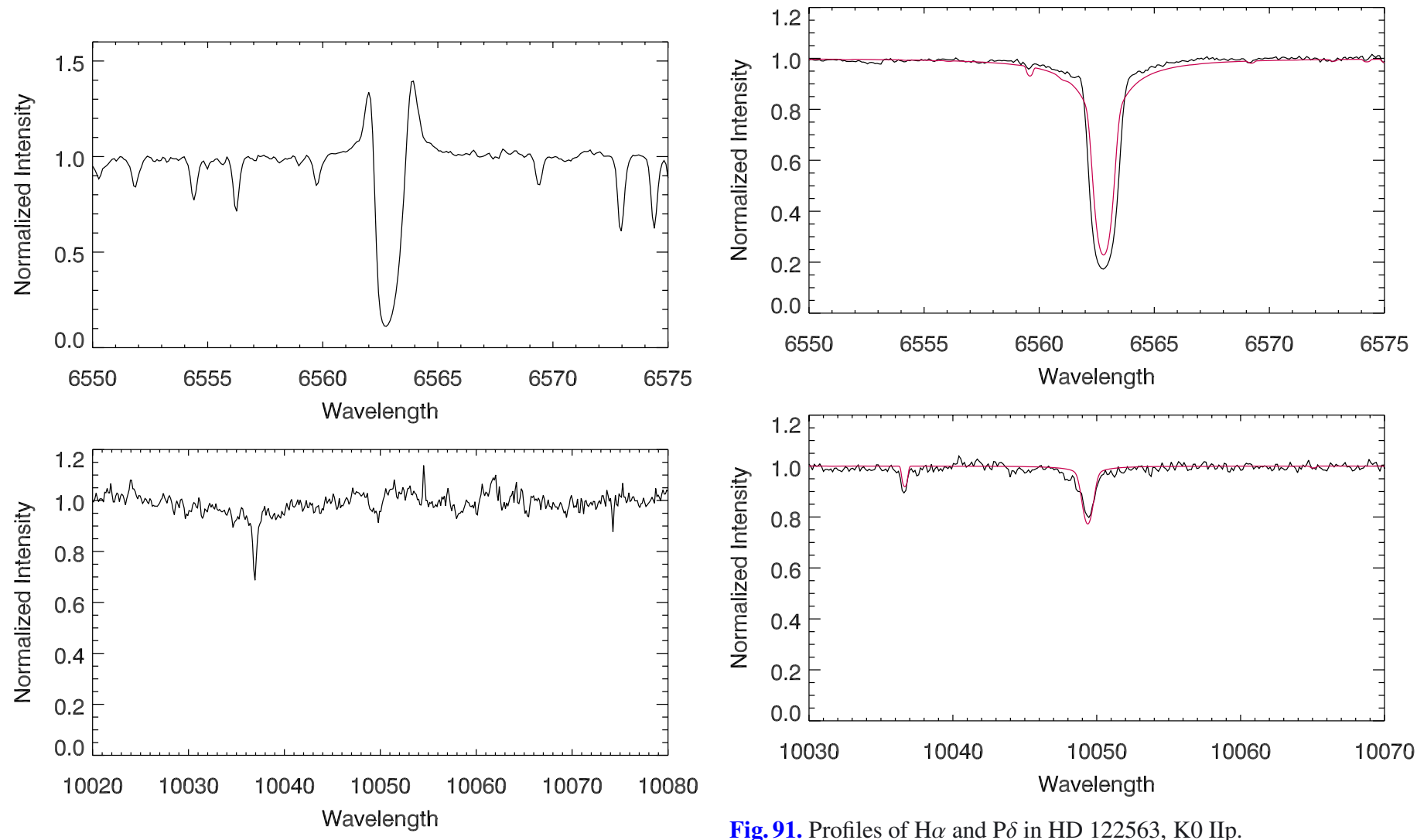

Fig. 89. Profiles of $\mathrm{H} \alpha$ and $\mathrm{P} \delta$ in $\mathrm{HD} 218732, \mathrm{G} 7 \mathrm{Ib}$.

Fig. 91. Profiles of $\mathrm{H} \alpha$ and $\mathrm{P} \delta$ in HD 122563, K0 IIp. 
A\&A 547, A62 (2012)
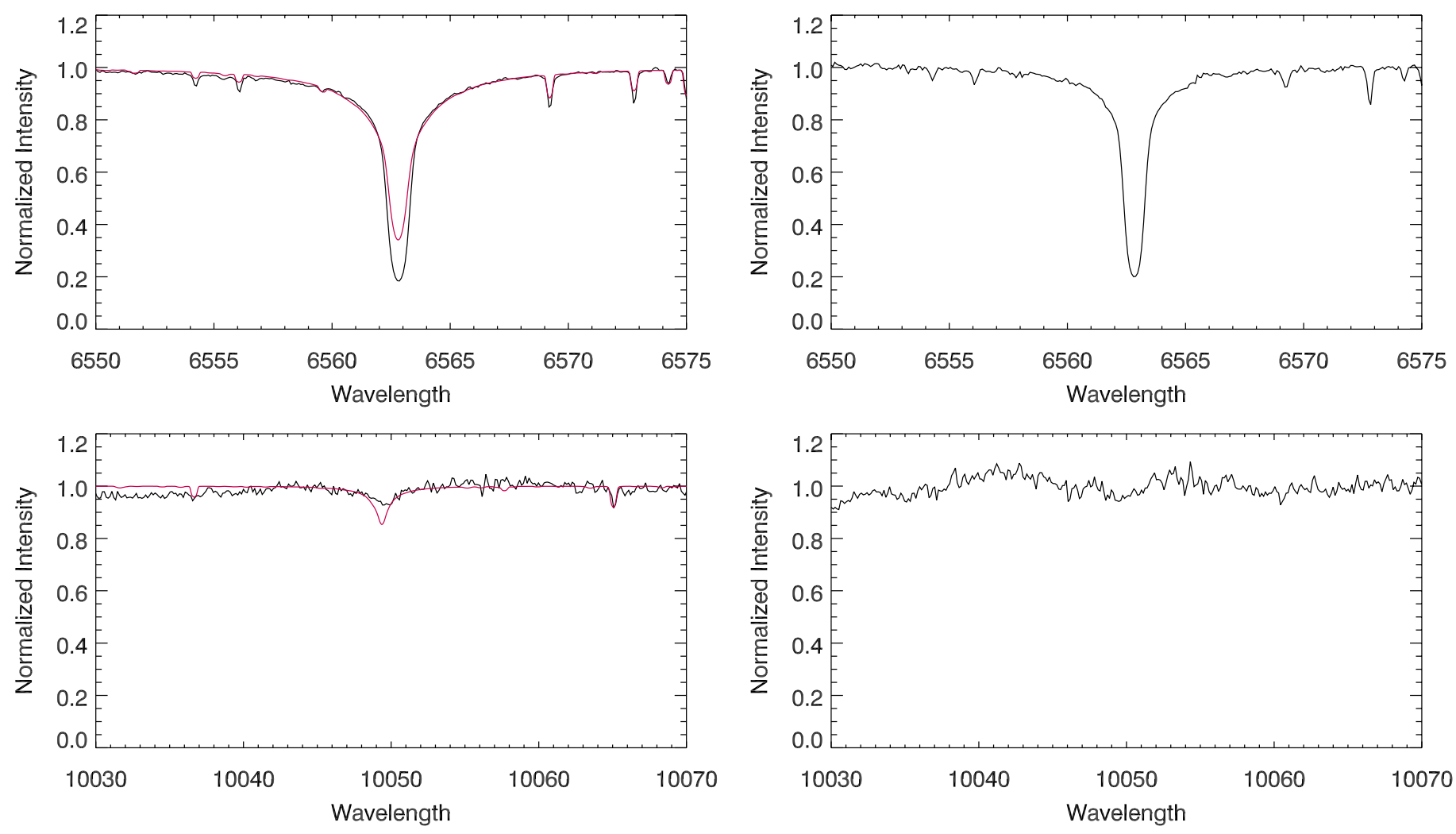

Fig. 92. Profiles of $\mathrm{H} \alpha$ and $\mathrm{P} \delta$ in $\mathrm{HD} 103095, \mathrm{~K} 0 \mathrm{Vp}$.

Fig. 94. Profiles of $\mathrm{H} \alpha$ and $\mathrm{P} \delta$ in $\mathrm{HD} 25329, \mathrm{~K} 1 \mathrm{~V}$.
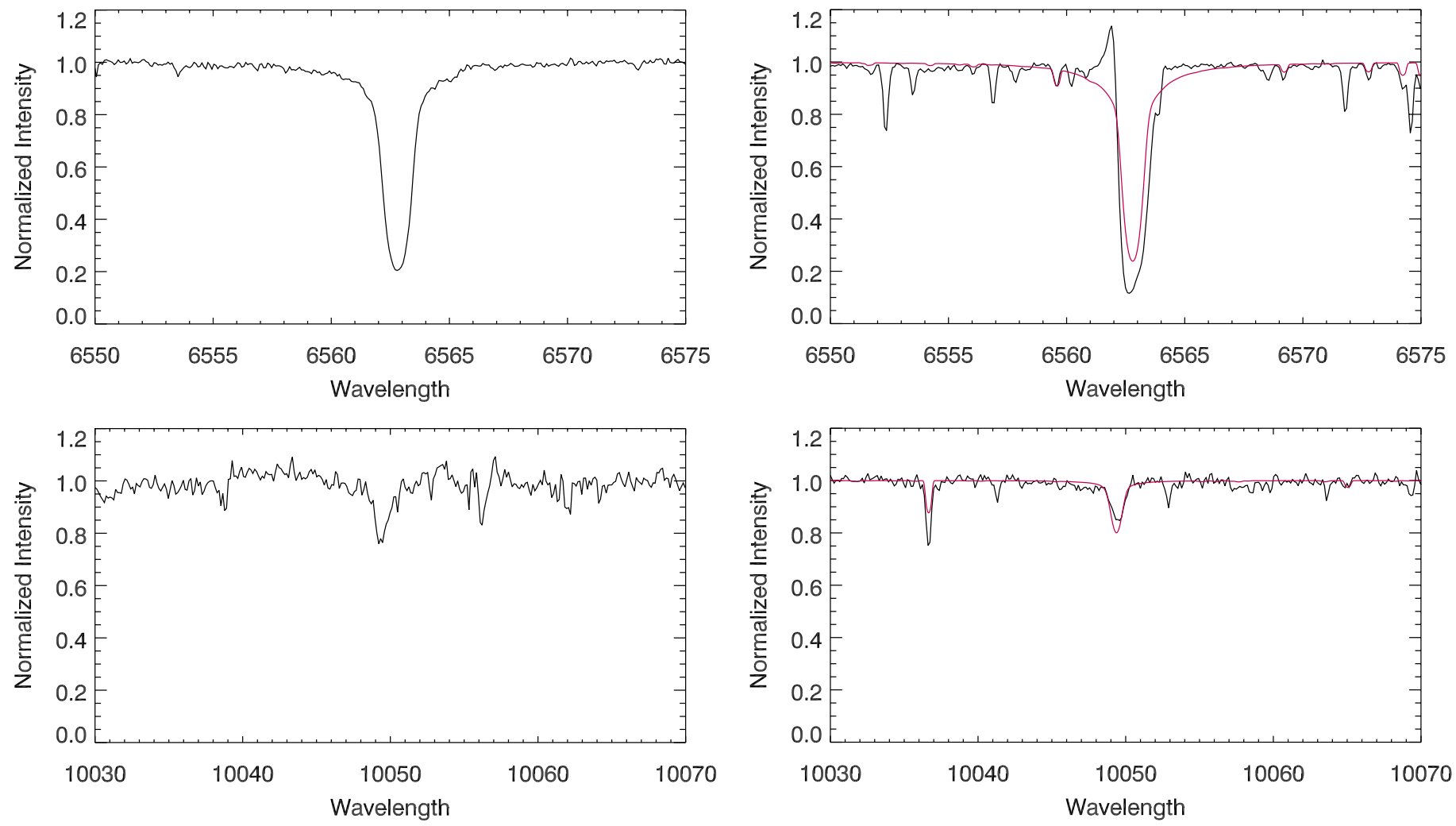

Fig. 93. Profiles of $\mathrm{H} \alpha$ and $\mathrm{P} \delta$ in $\mathrm{HD} 4306, \mathrm{~K}$ IIp.

Fig. 95. Profiles of $\mathrm{H} \alpha$ and $\mathrm{P} \delta$ in $\mathrm{HD} 165195, \mathrm{~K} 3 \mathrm{p}$. 
W. Huang et al.: A catalogue of Paschen-line profiles
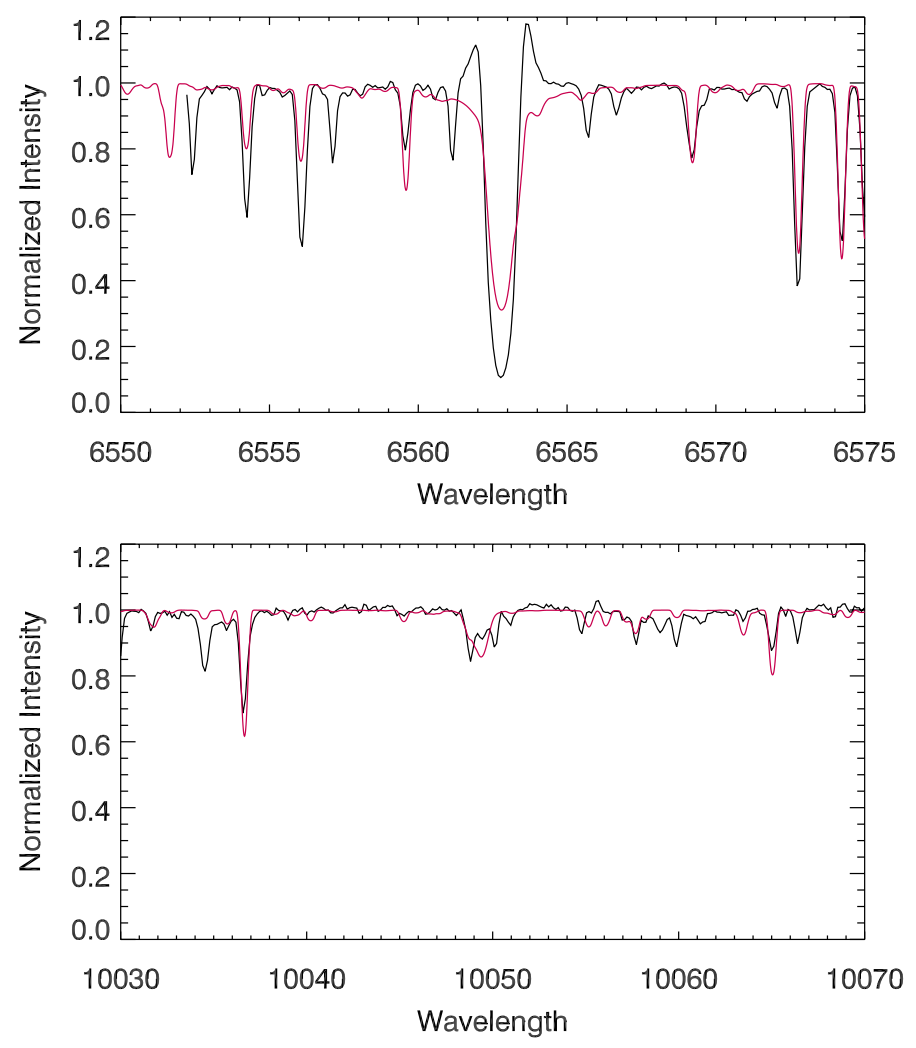

Fig. 96. Profiles of $\mathrm{H} \alpha$ and $\mathrm{P} \delta$ in HD 232078, K3 IIp. 